\title{
Imprints of relic gravitational waves in cosmic microwave background radiation
}

\author{
D. Baskaran \\ School of Physics and Astronomy, Cardiff University, Cardiff CF24 3YB, United Kingdom \\ L. P. Grishchuk \\ School of Physics and Astronomy, Cardiff University, Cardiff CF24 3YB, United Kingdom \\ and Sternberg Astronomical Institute, Moscow State University, Moscow 119899, Russia
}

A. G. Polnarev

Astronomy Unit, School of Mathematical Sciences Queen Mary, University of London, Mile End Road, London E1 4NS, United Kingdom

(Received 18 May 2006; published 27 October 2006)

\begin{abstract}
A strong variable gravitational field of the very early Universe inevitably generates relic gravitational waves by amplifying their zero-point quantum oscillations. We begin our discussion by contrasting the concepts of relic gravitational waves and inflationary "tensor modes". We explain and summarize the properties of relic gravitational waves that are needed to derive their effects on cosmic microwave background $(\mathrm{CMB})$ temperature and polarization anisotropies. The radiation field is characterized by four invariants $I, V, E, B$. We reduce the radiative transfer equations to a single integral equation of Voltairre type and solve it analytically and numerically. We formulate the correlation functions $C_{\ell}^{X X^{\prime}}$ for $X, X^{\prime}=T$, $E, B$ and derive their amplitudes, shapes and oscillatory features. Although all of our main conclusions are supported by exact numerical calculations, we obtain them, in effect, analytically by developing and using accurate approximations. We show that the $T E$ correlation at lower $\ell$ 's must be negative (i.e. an anticorrelation), if it is caused by gravitational waves, and positive if it is caused by density perturbations. This difference in $T E$ correlation may be a signature more valuable observationally than the lack or presence of the $B B$ correlation, since the $T E$ signal is about 100 times stronger than the expected $B B$ signal. We discuss the detection by WMAP of the $T E$ anticorrelation at $\ell \approx 30$ and show that such an anticorrelation is possible only in the presence of a significant amount of relic gravitational waves (within the framework of all other common assumptions). We propose models containing considerable amounts of relic gravitational waves that are consistent with the measured $T T, T E$ and $E E$ correlations.
\end{abstract}

DOI: 10.1103/PhysRevD.74.083008

PACS numbers: 98.70.Vc, 04.30.-w, 98.80.Cq

\section{INTRODUCTION}

The detection of primordial gravitational waves is rightly considered a highest priority task for the upcoming observational missions [1]. Relic gravitational waves are inevitably generated by strong variable gravitational field of the very early Universe. The generating mechanism is the superadiabatic (parametric) amplification of the waves' zero-point quantum oscillations [2]. In contrast to other known massless particles, the coupling of gravitational waves to the "external" gravitational field is such that they could be amplified or generated in a homogeneous isotropic FLRW (Friedmann-Lemaitre-Robertson-Walker) universe. This conclusion, at the time of its formulation, was on a collision course with the dominating theoretical doctrine. At that time, it was believed that the gravitational waves could not be generated in a FLRW universe, and the possibility of their generation required the early Universe to be strongly anisotropic (see, for example, [3]).

The generating mechanism itself relies only on the validity of general relativity and quantum mechanics. But the amount and spectral content of relic gravitational waves depend on a specific evolution of the cosmological scale factor (classical "pumping" gravitational field) $a(\eta)$.
The theory was applied to a variety of $a(\eta)$, including those that are now called inflationary models (for a sample of possible spectra of relic gravitational waves, see Fig. 4 in Ref. [4]). If a unique $a(\eta)$ were known in advance from some fundamental "theory of everything", we would have derived the properties of the today's signal with no ambiguity. In the absence of such a theory, we have to use the available partial information in order to reduce the number of options. This allows us to evaluate the level of the expected signals in various frequency intervals. The prize is very high - the actual detection of a particular background of relic gravitational waves will provide us with the unique clue to the "birth" of the Universe and its very early dynamical behavior.

A crucial assumption that we make in this and previous studies is that the observed large-scale cosmic microwave background (CMB) anisotropies are caused by cosmological perturbations of quantum-mechanical origin. If this is true, then general relativity and quantum mechanics tell us that relic gravitational waves should be a significant, if not a dominant, contributor to the observed large-scale anisotropies. From the existing data on the amplitude and spectrum of the CMB fluctuations we infer the amplitude and spectral slope of the very long relic gravitational waves. 
We then derive detailed predictions for indirect and direct observations of relic gravitational waves in various frequency bands.

At this point, it is important to clarify the difference between the concepts of relic gravitational waves and what is now called inflationary gravitational waves. The statements about inflationary gravitational waves ("tensor modes") are based on the inflation theory. This theory assumes that the evolution of the very early Universe was driven by a scalar field, coupled to gravity in a special manner. The theory does not deny the correctness of the previously performed calculations for relic gravitational waves. However, the inflationary theory proposes its own way of calculating the generation of density perturbations ("scalar modes"). The inflationary theory appeals exactly to the same mechanism of superadiabatic (parametric) amplification of quantum vacuum fluctuations, that is responsible for the generation of relic gravitational waves, but enforces very peculiar initial conditions in the scalar modes calculations.

According to the inflationary initial conditions, the amplitudes of the 'gauge-invariant' metric perturbations $\zeta$ associated with the scalar modes (or, in other words, the amplitudes of the curvature perturbations called $\zeta$ or $\mathcal{R}$ ) can be arbitrarily large from the very beginning of their evolution. Moreover, the theory demands that these amplitudes must be infinitely large in the limit of the deSitter expansion law $a(\eta) \propto|\eta|^{-1}$ which is responsible for the generation of a flat (Harrison-Zeldovich-Peebles, 'scaleinvariant') primordial spectrum, with the spectral index $\mathrm{n}=1$. At the same time, the amplitudes of the generated gravitational waves are finite and small for all spectral indices, including $\mathrm{n}=1$. Since both, gravitational waves and density perturbations, produce CMB anisotropies and we see them small today, the inflationary theory substitutes (for "consistency") its prediction of infinitely large amplitudes of density perturbations, in the limit $\mathrm{n}=1$, by the claim that it is the amount of primordial gravitational waves, expressed in terms of the 'tensor/scalar ratio $r^{\prime}$, that should be zero, $r=0$. For a detailed critical analysis of the inflationary conclusions, see [5]; for arguments aimed at defending those conclusions, see [6].

The science motivations and CMB data analysis pipelines, designed to evaluate the gravitational-wave contribution, are usually based on inflationary formulas [7-10]. In particular, according to the inflation theory, the primordial power spectrum of density perturbations has the form (it follows, for example, from Eqs. (2.12a) and (2.12b) in Ref. [10] or from Eqs. (18) and (19) (or A12 and A13) in Ref. [7]):

$$
P_{S}(k)=\left.\frac{1}{4 \pi^{3} M_{\mathrm{Pl}}^{2}} \frac{1}{r} H^{2}\right|_{(k=a H)} .
$$

Despite the fact that this spectrum diverges at $r=0\left(\mathrm{n}_{s}=\right.$ $1, \mathrm{n}_{t}=0$ ), the CMB data analysts persistently claim that the inflation theory is in spectacular agreement with observations and the CMB data are perfectly well consistent with $r=0$ (the published confidence level contours always include $r=0$ and are typically centered at that point).

Our analysis in this paper, based on general relativity and quantum mechanics, is aimed at showing that there is evidence of signatures of relic gravitational waves in the already available CMB data. We also make predictions for some future experiments and observations.

The plan of the paper is as follows. In Sec. II we summarize the properties of a random background of relic gravitational waves that are needed for CMB calculations. The emphasis is on the gravitational wave (g.w.) mode functions, power spectra, and statistical relations. In Sec. III we discuss the general equations of radiative transfer and explain the existence of four invariants $I, V, E, B$ that fully characterize the radiation field. We formulate the linearized equations in the presence of a single Fourier mode of gravitational waves. We prove that there exists a choice of variables that reduces the problem of temperature and polarization anisotropies to only two functions of time $\alpha(\eta)$ and $\beta(\eta)$.

Section IV is devoted to further analysis of the radiative transfer equations. The main result of this section is the reduction of coupled integro-differential equations to a single integral equation of Voltairre type. Essentially, the entire problem of the CMB polarization is reduced to a single integral equation. This allows us to use simple analytical approximations and give transparent physical interpretation. In Sec. V we generalize the analysis to a superposition of random Fourier modes with arbitrary wave vectors. We derive (and partially rederive the previously known) expressions for multipole coefficients $a_{\ell m}^{X}$ of the radiation field. We show that the statistical properties of the multipole coefficients are fully determined by the statistical properties of the underlying gravitational perturbations. This section contains the expressions for general correlation and cross-correlation functions $C_{\ell}^{X X^{\prime}}$ for invariants of the radiation field.

We work out astrophysical applications in Secs. VI and VII where we discuss the effects of recombination and reionization era, respectively. Although all our main conclusions are supported by exact numerical calculations, we show the origin of these conclusions and essentially derive them by developing and using semianalytical approximations. The expected amplitudes, shapes, oscillatory features, etc. of all correlation functions as functions of $\ell$ are under analytical control. The central point of this analysis is the $T E$ correlation function. We show that, at lower multipoles $\ell$, the $T E$ correlation function must be negative (anticorrelation), if it is induced by gravitational waves, and positive, if it is induced by density perturbations. We argue that this difference in sign of $T E$ correlations can be a signature more valuable observationally than the presence or absence of the $B B$ correlations. This is 
because the $T E$ signal is about 2 orders of magnitude larger than the expected $B B$ signal and is much easier to measure. We summarize the competing effects of density perturbations in Appendix D.

Theoretical findings are compared with observations in Sec. VIII. In the context of relic gravitational waves it is especially important that the WMAP team [9] stresses (even if for a different reason) the actual detection of the $T E$ anticorrelation near $\ell \approx 30$. We show that this is possible only in the presence of a significant amount of relic gravitational waves (within the framework of all other common assumptions). We analyze the CMB data and suggest models with significant amounts of gravitational waves that are consistent with the measured $T T, T E$ and $E E$ correlation functions. Our final conclusion is that there is evidence of the presence of relic gravitational waves in the already available CMB data, and further study of the $T E$ correlation at lower $\ell$ 's has the potential of a firm positive answer.

\section{GENERAL PROPERTIES OF RELIC GRAVITATIONAL WAVES}

Here we summarize some properties of cosmological perturbations of quantum-mechanical origin. We will need formulas from this summary for our further calculations.

\section{A. Basic definitions}

As usual (for more details, see, for example, [11]), we write the perturbed gravitational field of a flat FLRW universe in the form

$$
\begin{aligned}
d s^{2} & =-c^{2} d t^{2}+a^{2}(t)\left(\delta_{i j}+h_{i j}\right) d x^{i} d x^{j} \\
& =a^{2}(\eta)\left[-d \eta^{2}+\left(\delta_{i j}+h_{i j}\right) d x^{i} d x^{j}\right] .
\end{aligned}
$$

We denote the present moment of time by $\eta=\eta_{R}$ and define it by the observed quantities, for example, by today's value of the Hubble parameter $H_{0}=H\left(\eta_{R}\right)$. In addition, we take the present-day value of the scale factor to be $a\left(\eta_{R}\right)=2 l_{H}$, where $l_{H}=c / H_{0}$.

The functions $h_{i j}(\eta, \mathbf{x})$ are expanded over spatial Fourier harmonics $e^{ \pm i \mathbf{n} \cdot \mathbf{x}}$, where $\mathbf{n}$ is a dimensionless time independent wave vector. The wave number $n$ is $n=$ $\left(\delta_{i j} n^{i} n^{j}\right)^{1 / 2}$. The wavelength $\lambda$, measured in units of laboratory standards, is related to $n$ by $\lambda=2 \pi a / n$. The waves whose wavelengths today are equal to today's Hubble radius carry the wave number $n_{H}=4 \pi$. Shorter waves have larger $n$ 's and longer waves have smaller $n$ 's.

The often used dimensional wave number $k$, defined by $k=2 \pi / \lambda\left(\eta_{R}\right)$ in terms of today's wavelength $\lambda\left(\eta_{R}\right)$, is related to $n$ by a simple formula

$$
k=\frac{n}{2 l_{H}} \approx n\left(1.66 \times 10^{-4} h\right) \mathrm{Mpc}^{-1} .
$$

The expansion of the field $h_{i j}(\eta, \mathbf{x})$ over Fourier components $\mathbf{n}$ requires a specification of polarization tensors $\stackrel{s}{p}_{i j}(\mathbf{n})(\mathrm{s}=1,2)$. They have different forms depending on whether the functions $h_{i j}(\eta, \mathbf{x})$ represent gravitational waves, rotational perturbations, or density perturbations.

In the case of gravitational waves, two independent linear polarization states can be described by two real polarization tensors

$$
\stackrel{1}{p}_{i j}(\mathbf{n})=l_{i} l_{j}-m_{i} m_{j}, \quad \stackrel{2}{p}_{i j}(\mathbf{n})=l_{i} m_{j}+m_{i} l_{j},
$$

where spatial vectors $(\boldsymbol{l}, \mathbf{m}, \mathbf{n} / n)$ are unit and mutually orthogonal vectors. The polarization tensors (2) satisfy the conditions

$$
\stackrel{s}{p}_{i j} \delta^{i j}=0, \quad \stackrel{s}{p}_{i j} n^{i}=0, \quad \stackrel{s}{p}_{i j} p^{i j}=2 \delta_{s^{\prime} s} .
$$

The eigenvectors of $\stackrel{1}{p}_{i j}$ are $l_{i}$ and $m_{i}$, whereas the eigenvectors of $\stackrel{2}{p}_{i j}$ are $l_{i}+m_{i}$ and $l_{i}-m_{i}$. In both cases, the first eigenvector has the eigenvalue +1 , whereas the second eigenvector has the eigenvalue -1 .

In terms of spherical coordinates $\theta, \phi$, we choose for $(\boldsymbol{l}, \mathbf{m}, \mathbf{n} / n)$ the right-handed triplet:

$$
\begin{aligned}
\boldsymbol{l} & =(\cos \theta \cos \phi, \cos \theta \sin \phi,-\sin \theta), \\
\mathbf{m} & =(-\sin \phi, \cos \phi, 0), \\
\mathbf{n} / n & =(\sin \theta \cos \phi, \sin \theta \sin \phi, \cos \theta) .
\end{aligned}
$$

The vector $\boldsymbol{l}$ points along a meridian in the direction of increasing $\theta$, while the vector $\mathbf{m}$ points along a parallel in the direction of increasing $\phi$. With this specification, polarization tensors (2) will be called the ' + ' and ' $X$ ' polarizations. The eigenvectors of ' + ' polarization correspond to north-south and east-west directions, whereas the ' $X$ ' polarization describes the directions rotated by $45^{\circ}$.

With a fixed $\mathbf{n}$, the choice of vectors $\boldsymbol{l}, \mathbf{m}$ given by Eq. (4) is not unique. The vectors can be subject to continuous and discrete transformations. The continuous transformation is performed by a rotation of the pair $\boldsymbol{l}, \mathbf{m}$ in the plane orthogonal to $\mathbf{n}$ :

$$
\boldsymbol{l}^{\prime}=\boldsymbol{l} \cos \psi+\mathbf{m} \sin \psi, \quad \mathbf{m}^{\prime}=-\boldsymbol{l} \sin \psi+\mathbf{m} \cos \psi,
$$

where $\psi$ is an arbitrary angle. The discrete transformation is described by the flips of the $\boldsymbol{l}, \mathbf{m}$ vectors:

$$
\boldsymbol{l}^{\prime}=-\boldsymbol{l}, \quad \mathbf{m}^{\prime}=\mathbf{m} \quad \text { or } \quad \boldsymbol{l}^{\prime}=\boldsymbol{l}, \quad \mathbf{m}^{\prime}=-\mathbf{m} .
$$

When (5) is applied, polarization tensors (2) transform as

$$
\begin{aligned}
& \stackrel{1}{p}_{i j}^{\prime}(\mathbf{n})=l_{i}^{\prime} l_{j}^{\prime}-m_{i}^{\prime} m_{j}^{\prime}=\stackrel{1}{p}_{i j}(\mathbf{n}) \cos 2 \psi+\stackrel{2}{p}_{i j}(\mathbf{n}) \sin 2 \psi, \\
& \stackrel{2}{p}_{i j}^{\prime}(\mathbf{n})=l_{i}^{\prime} m_{j}^{\prime}+m_{i}^{\prime} l_{j}^{\prime}=-\stackrel{1}{p}_{i j}(\mathbf{n}) \sin 2 \psi+\stackrel{2}{p}_{i j}(\mathbf{n}) \cos 2 \psi,
\end{aligned}
$$

and when (6) is applied they transform as 


$$
\stackrel{1}{p}_{i j}^{\prime}(\mathbf{n})=\stackrel{1}{p}_{i j}(\mathbf{n}), \quad \stackrel{2}{p}_{i j}^{\prime}(\mathbf{n})=-\stackrel{2}{p}_{i j}(\mathbf{n}) .
$$

Later in this section and in Appendix A, we are discussing the conditions under which the averaged observational properties of a random field $h_{i j}(\mathbf{n}, \eta)$ are symmetric with respect to rotations around the axis $\mathbf{n} / n$ and with respect to mirror reflections of the axes. Formally, this is expressed as the requirement of symmetry of the g.w. field correlation functions with respect to transformations (5) and (6).

In this paper we will also be dealing with density perturbations. In this case, the polarization tensors are

$$
\stackrel{1}{p}_{i j}=\sqrt{\frac{2}{3}} \delta_{i j}, \quad \stackrel{2}{p}_{i j}=-\sqrt{3} \frac{n_{i} n_{j}}{n^{2}}+\frac{1}{\sqrt{3}} \delta_{i j} .
$$

These polarization tensors satisfy the last of the conditions (3).

In the rigorous quantum-mechanical version of the theory, the functions $h_{i j}$ are quantum-mechanical operators. We write them in the form:

$$
\begin{aligned}
h_{i j}(\eta, \mathbf{x})= & \frac{\mathcal{C}}{(2 \pi)^{3 / 2}} \int_{-\infty}^{+\infty} d^{3} \mathbf{n} \frac{1}{\sqrt{2 n}} \\
& \times \sum_{s=1,2}\left[\stackrel{s}{p_{i j}}(\mathbf{n}) \stackrel{s}{h}{ }_{n}(\eta) e^{i \mathbf{n} \cdot \mathbf{x}}{ }_{c_{\mathbf{n}}}\right. \\
& \left.+\stackrel{s}{p_{i j}}{ }^{*}(\mathbf{n}) \stackrel{s}{h_{n}^{*}}{ }^{*}(\eta) e^{-i \mathbf{n} \cdot \mathbf{x}^{s}} c_{\mathbf{n}}^{\dagger}\right],
\end{aligned}
$$

where the annihilation and creation operators, $\stackrel{s}{c_{\mathbf{n}}}$ and $\stackrel{s}{c_{\mathbf{n}}^{\dagger}}$, satisfy the relationships

$$
\left[{\stackrel{s}{s^{\prime}}}_{c_{\mathbf{n}}},{\stackrel{c^{\dagger}}{\mathbf{n}^{\prime}}}^{\dagger}=\delta_{s^{\prime} s} \delta^{(3)}\left(\mathbf{n}-\mathbf{n}^{\prime}\right), \quad{ }^{s} \mathbf{c}_{\mathbf{n}}|0\rangle=0\right.
$$

The initial vacuum state $|0\rangle$ of perturbations is defined at some moment of time $\eta_{0}$ in the remote past, long before the onset of the process of superadiabatic amplification. This quantum state is maintained (in the Heisenberg picture) until now. For gravitational waves, the normalization constant is $\mathcal{C}=\sqrt{16 \pi} l_{\mathrm{Pl}}$.

The relationships (11) determine the expectation values and correlation functions of cosmological perturbations themselves, and also of the CMB's temperature and polarization anisotropies caused by these cosmological perturbations. In particular, the variance of metric perturbations is given by

$$
\left\langle 0\left|h_{i j}(\eta, \mathbf{x}) h^{i j}(\eta, \mathbf{x})\right| 0\right\rangle=\frac{\mathcal{C}^{2}}{2 \pi^{2}} \int_{0}^{\infty} n^{2} \sum_{s=1,2}\left|h_{n}(\eta)\right|^{2} \frac{d n}{n} .
$$

The quantity

$$
h^{2}(n, \eta)=\frac{\mathcal{C}^{2}}{2 \pi^{2}} n^{2} \sum_{s=1,2}\left|\stackrel{s}{h}_{n}(\eta)\right|^{2}=\frac{1}{2} \sum_{s=1,2}|s h(n, \eta)|^{2},
$$

is called the metric power spectrum. Note that we have introduced

$$
\stackrel{s}{h}(n, \eta)=\frac{\mathcal{C}}{\pi} n \stackrel{s}{h}(\eta)
$$

The quantity (13) gives the mean-square value of the gravitational-field perturbations in a logarithmic interval of $n$. The spectrum of the root-mean-square (rms) amplitude $h(n, \eta)$ is determined by the square root of Eq. (13). Having evolved the classical mode functions ${ }_{h} h_{n}(\eta)$ up to some arbitrary instant of time $\eta$ (for instance, today $\eta=$ $\eta_{R}$ ) one can find the power spectrum $h(n, \eta)$ at that instant of time. For the today's spectrum in terms of frequency $\nu$ measured in $\mathrm{Hz}, \nu=n H_{0} / 4 \pi$, we use the notation $h_{\mathrm{rms}}(\nu)$.

In our further applications we will also need the power spectrum of the first time-derivative of metric perturbations:

$$
\begin{gathered}
\left\langle 0\left|\frac{\partial h_{i j}(\eta, \mathbf{x})}{\partial \eta} \frac{\partial h^{i j}(\eta, \mathbf{x})}{\partial \eta}\right| 0\right\rangle \\
=\frac{1}{2} \int_{0}^{\infty} \sum_{s=1,2}\left|\frac{d h(n, \eta)}{d \eta}\right|^{2} \frac{d n}{n} .
\end{gathered}
$$

To simplify calculations, in the rest of the paper we will be using a "classical" version of the theory, whereby the quantum-mechanical operators $\stackrel{s}{c_{\mathbf{n}}}$ and $\stackrel{s \dagger}{c_{\mathbf{n}}^{\dagger}}$ are treated as classical random complex numbers $\stackrel{s}{c_{\mathbf{n}}}$ and ${ }^{s^{*}} c_{\mathbf{n}}$. It is assumed that they satisfy the relationships analogous to (11):

$$
\begin{aligned}
& \left\langle\stackrel{s}{c_{\mathbf{n}}}\right\rangle=\left\langle s^{s^{\prime *}} c_{\mathbf{n}^{\prime}}\right\rangle=0,
\end{aligned}
$$

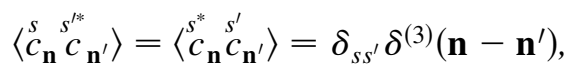

$$
\begin{aligned}
& \left\langle\begin{array}{ll}
s & s^{\prime} \\
c_{\mathbf{n}} & c_{\mathbf{n}^{\prime}}
\end{array}\right\rangle=\left\langle\begin{array}{ll}
s^{*} & s^{\prime *} \\
c_{\mathbf{n}} & c_{\mathbf{n}^{\prime}}
\end{array}\right\rangle=0,
\end{aligned}
$$

where the averaging is performed over the ensemble of all possible realizations of the random field (10).

The relationships (16) are the only statistical assumptions that we make. They fully determine all the expectations values and correlation functions that we will calculate, both for cosmological perturbations and for the induced CMB fluctuations. For example, the metric power spectrum (13) follows now from the calculation:

$$
\frac{1}{2}\left\langle h_{i j}(\eta, \mathbf{x}) h^{i j}(\eta, \mathbf{x})\right\rangle=\frac{\mathcal{C}^{2}}{2 \pi^{2}} \int_{0}^{\infty} n^{2} \sum_{s=1,2}\left|h_{n}(\eta)\right|^{2} \frac{d n}{n} .
$$

The quantities $\left|h_{n}(\eta)\right|^{2}$ are responsible for the magnitude of the mean-square fluctuations of the field in the corresponding polarization states $s$. In general, the assumption of statistical independence of two linear polarization components in one polarization basis is not equivalent to this assumption in another polarization basis. As we show in Appendix A, statistical properties are independent of the basis (i.e. independent of $\psi$, Eq. (7)), if the condition 


$$
\left|\stackrel{+}{h}_{n}(\eta)\right|^{2}=\left|\stackrel{\times}{h}_{n}(\eta)\right|^{2}
$$

is satisfied. As for the discrete transformations (8), they leave the g.w. field correlation functions unchanged.

In our further discussion of the CMB polarization it will be convenient to use also the expansion of $h_{i j}$ over circular, rather than linear, polarization states. In terms of definitions (2), the left $(s=L)$ and right $(s=R)$ circular polarization states are described by the complex polarization tensors

$$
\begin{array}{cl}
\stackrel{L}{p}_{i j}=\frac{1}{\sqrt{2}}\left(\stackrel{1}{p}_{i j}+\stackrel{2}{p}_{i j}\right), & \stackrel{R}{p_{i j}}=\frac{1}{\sqrt{2}}\left({ }_{p} p_{i j}-i_{i j}^{2} p_{i j}\right), \\
\stackrel{L}{p}_{i j}{ }^{*}=\stackrel{R}{p}_{i j}, & \stackrel{R}{p}_{i j}{ }^{*}=\stackrel{L}{p}_{i j},
\end{array}
$$

satisfying the conditions (for $s=L, R$ )

$$
\stackrel{s}{p}_{i j} \delta^{i j}=0, \quad \stackrel{s}{p}_{i j} n^{i}=0, \quad \stackrel{s}{p}_{i j}^{\prime} p^{i j^{*}}=2 \delta_{s^{\prime} s} .
$$

A continuous transformation (5) brings the tensors (19) to the form

$$
\stackrel{L}{p}_{i j}^{\prime}=\stackrel{L}{p}_{i j} e^{-i 2 \psi}, \quad \stackrel{R}{p}_{i j}^{\prime}=\stackrel{R}{p}_{i j} e^{i 2 \psi} .
$$

Functions transforming according to the rule (21) are called the spin-weighted functions of spin +2 and spin -2 , respectively [12-14]. A discrete transformation (6) applied to (19) interchanges the left and right polarization states:

$$
\stackrel{L}{p}^{\prime}{ }_{i j}=\stackrel{R}{p}_{i j}, \quad \stackrel{R}{p}_{i j}^{\prime}=\stackrel{L}{p}_{i j} .
$$

The assumption of statistical independence of two linear polarization states is, in general, not equivalent to the assumption of statistical independence of two circular polarization states. Moreover, it is shown in Appendix A that symmetry between left and right is violated, unless

$$
\left|h_{n}(\eta)\right|^{2}=\left|h_{n}^{R}(\eta)\right|^{2} .
$$

However, if conditions (18) and (23) are satisfied, statistical properties of the random g.w. field remain unchanged under transformations from one basis to another, including the transitions between linear and circular polarizations. The summation over $s$ in the power spectra such as (17) can be replaced by the multiplicative factor 2 . Moreover, the mode functions ${ }_{h} h_{n}(\eta)$ for two independent polarization states will be equal up to a constant complex factor $e^{i \alpha}$. This factor can be incorporated in the redefinition of random coefficients $\stackrel{s}{c_{\mathbf{n}}}$ without violating the statistical assumptions (16). After this, the index $s$ over the mode functions can be dropped:

$$
\stackrel{s}{h}_{n}(\eta)=h_{n}(\eta)
$$

There is no special reason for the quantum-mechanical generating mechanism to prefer one polarization state over another. It is natural to assume that the conditions (18) and (23) hold true for relic gravitational waves, but in general they could be violated. In calculations below we often use these equalities, but we do not enforce them without warning.

\section{B. Mode functions and power spectra}

The perturbed Einstein equations give rise to the g.w. equation for the mode functions $h_{n}(\eta)$. This equation can be transformed to the equation for a parametrically disturbed oscillator [2]:

$$
\stackrel{s}{\mu}_{n}+\stackrel{s}{\mu}_{n}\left[n^{2}-\frac{a^{\prime \prime}}{a}\right]=0,
$$

where $\stackrel{s}{\mu}_{n}(\eta) \equiv a(\eta) \stackrel{s}{h}(\eta)$ and ${ }^{\prime}=d / d \eta=(a / c) d / d t$. (In this paper, we ignore anisotropic stresses. For the most recent account of this subject, which includes earlier references, see [15].) Clearly, the behavior of the mode functions depend on the gravitational "pumping" field $a(\eta)$, regardless of the physical nature of the matter sources driving the cosmological scale factor $a(\eta)$. Observational data about relic gravitational waves allow us to make direct inferences about $H(\eta)$ and $a(\eta)$ [16], and it is only through extra assumptions that we can make inferences about such things as, say, the scalar field potential (if it is relevant at all).

Previous analytical calculations (see, for example, $[11,17]$ and references there) were based on models where $a(\eta)$ consists of pieces of power-law evolution

$$
a(\eta)=l_{o}|\eta|^{1+\beta},
$$

where $l_{o}$ and $\beta$ are constants. The functions $a(\eta), a^{\prime}(\eta)$, $h_{n}(\eta),{ }_{h}^{s}{ }^{\prime}(\eta)$ were continuously joined at the transition points between various power-law eras.

It is often claimed in the literature that this method of joining the solutions is unreliable, unless the wavelength is "much longer than the time taken for the transition to take place". Specifically, it is claimed that the joining procedure leads to huge errors in the g.w. power spectrum for short waves. It is important to show that these claims are incorrect. As an example, we will consider the transition between the radiation-dominated and matter-dominated eras.

The exact scale factor, which accounts for the simultaneous presence of matter, $\rho_{m} \propto a(\eta)^{-3}$, and radiation, $\rho_{\gamma} \propto a(\eta)^{-4}$, has the form

$$
a(\eta)=2 l_{H}\left(\frac{1+z_{\mathrm{eq}}}{2+z_{\mathrm{eq}}}\right) \eta\left(\eta+\frac{2 \sqrt{2+z_{\mathrm{eq}}}}{1+z_{\mathrm{eq}}}\right),
$$

where $z_{\text {eq }}$ is the redshift of the era of equality of energy densities in matter and radiation $\rho_{m}\left(z_{\mathrm{eq}}\right)=\rho_{\gamma}\left(z_{\mathrm{eq}}\right)$, 


$$
1+z_{\mathrm{eq}}=\frac{a\left(\eta_{R}\right)}{a\left(\eta_{\mathrm{eq}}\right)}=\frac{\Omega_{m}}{\Omega_{\gamma}} .
$$

For this model, the values of parameter $\eta$ at equality and today are given by the expressions

$$
\begin{aligned}
& \eta_{\mathrm{eq}}=(\sqrt{2}-1) \frac{\sqrt{2+z_{\mathrm{eq}}}}{1+z_{\mathrm{eq}}}, \\
& \eta_{R}=1-\frac{\sqrt{2+z_{\mathrm{eq}}}}{1+z_{\mathrm{eq}}}+\frac{1}{1+z_{\mathrm{eq}}} .
\end{aligned}
$$

The current observations favor the value $z_{\mathrm{eq}} \approx 3 \times 10^{3}$ [18].

The piecewise approximation to the scale factor (27) has the form

$$
\begin{aligned}
& a(\eta)=\frac{4 l_{H}}{\sqrt{1+z_{\mathrm{eq}}}} \eta, \quad \eta \leq \eta_{\mathrm{eq}} ; \\
& a(\eta)=2 l_{H}\left(\eta+\eta_{\mathrm{eq}}\right)^{2}, \quad \eta \geq \eta_{\mathrm{eq}},
\end{aligned}
$$

where $\eta_{\mathrm{eq}}$ and $\eta_{R}$ for this joined $a(\eta)$ are given by $\eta_{\mathrm{eq}}=$ $1 / 2 \sqrt{1+z_{\mathrm{eq}}}$ and $\eta_{R}=1-1 / 2 \sqrt{1+z_{\mathrm{eq}}}$. It can be seen from (27) and (28) that the relative difference between the two scale factors is very small in the deep radiationdominated era, $\eta \ll 1 / \sqrt{z_{\mathrm{eq}}}$, and in the deep matterdominated era, $\eta \gg 1 / \sqrt{z_{\text {eq }}}$. But the difference reaches about $25 \%$ at times near equality.

The initial conditions for the g.w. Equation (25) are the same in the models (27) and (28) and they are determined by quantum-mechanical assumptions at the stage (which we call the $i$-stage) preceding the radiation epoch. The $i$-stage has finished, and the radiation-dominated stage has started, at some $\eta_{i}$ with a redshift $z_{i}$. The numerical value of $z_{i}$ should be somewhere near $10^{29}$ (see below).

The initial conditions at the radiation-dominated stage can be specified at that early time $\eta_{i}$ or, in practice, for numerical calculations, at much latter time, as long as the appropriate g.w. solution is taken as [11]

$$
\stackrel{s}{\mu}{ }_{n}(\eta)=-2 i B \sin n \eta, \quad \stackrel{s}{\mu}_{n}^{\prime}(\eta)=-2 i n B \cos n \eta,
$$

where

$$
B=F(\beta)\left(\frac{n \sqrt{1+z_{\mathrm{eq}}}}{1+z_{i}}\right)^{\beta}
$$

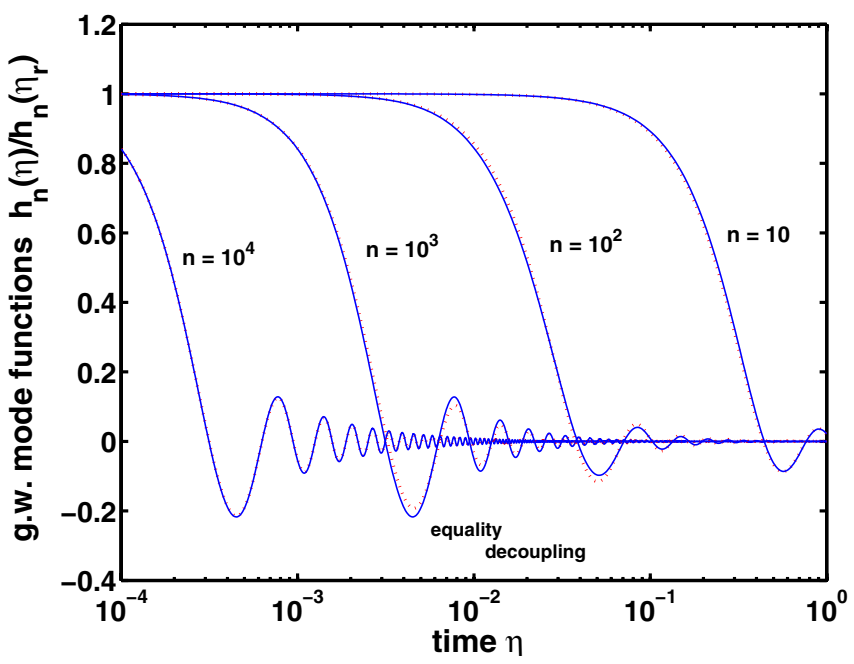

FIG. 1 (color online). The g.w. mode functions $h_{n}(\eta) / h_{n}\left(\eta_{r}\right)$ in a matter-radiation universe. The solid curves are numerically calculated solutions on the scale factor (27), while the dotted curves are analytical solutions on the scale factor (28).

and $F(\beta)$ is a slowly varying function of the constant parameter $\beta,|F(-2)|=2$. Parameter $\beta$ describes the power-law evolution at the $i$-stage and determines the primordial spectral index $\mathrm{n}, \mathrm{n}=2 \beta+5$. In particular, $\beta=-2$ corresponds to the flat (scale-invariant) primordial spectrum $\mathrm{n}=1$.

For numerical calculations we use the constant $B$ in the form:

$$
B=2\left(\frac{4 \pi \sqrt{1+z_{\mathrm{eq}}}}{1+z_{i}}\right)^{\beta}\left(\frac{n}{n_{H}}\right)^{\beta} .
$$

The wave-Eq. (25) with the scale factor (27) can not be solved in elementary functions. However, it can be solved numerically using the initial data (29). Concretely, we have imposed the initial data (29) at $\eta_{r}=10^{-6}$, which corresponds to the redshift $z \approx 3 \times 10^{7}$, and have chosen $z_{e q}=$ $6 \times 10^{3}$ for illustration. Numerical solutions for $h_{n}(\eta) / h_{n}\left(\eta_{r}\right)$ characterized by different wave numbers $n$ are shown by solid lines in Fig. 1. We should compare these solutions with the joined solutions found on the joined evolution (28) for the same wave numbers.

The piecewise scale factor (28) allows one to write down the piecewise analytical solutions to the g.w. Equation (25):

$$
\mu_{n}(\eta)= \begin{cases}-2 i B \sin n \eta, & \eta \leq \eta_{\mathrm{eq}} \\ \sqrt{n\left(\eta+\eta_{\mathrm{eq}}\right)}\left[A_{n} J_{3 / 2}\left(n\left(\eta+\eta_{\mathrm{eq}}\right)\right)-i B_{n} J_{-3 / 2}\left(n\left(\eta+\eta_{\mathrm{eq}}\right)\right)\right], & \eta \geq \eta_{\mathrm{eq}}\end{cases}
$$

where $J_{3 / 2}, J_{-3 / 2}$ are Bessel functions. The coefficients $A_{n}$ and $B_{n}$ are calculated from the condition of continuous joining of $\mu_{n}$ and $\mu_{n}^{\prime}$ at $\eta_{\mathrm{eq}}[11]$ : 


$$
\begin{aligned}
A_{n} & =-i \sqrt{\frac{\pi}{2}} \frac{B}{4 y_{2}^{2}}\left[\left(8 y_{2}^{2}-1\right) \sin y_{2}+4 y_{2} \cos y_{2}+\sin 3 y_{2}\right], \\
B_{n} & =-\sqrt{\frac{\pi}{2}} \frac{B}{4 y_{2}^{2}}\left[\left(8 y_{2}^{2}-1\right) \cos y_{2}-4 y_{2} \sin y_{2}+\cos 3 y_{2}\right],
\end{aligned}
$$

where $y_{2}=n \eta_{\text {eq }}$. In Fig. 1 we show the joined solutions (31) by dotted curves.

It is clear from Fig. 1 that the g.w. solutions as functions of $\eta$ are pretty much similar in the two models. The solid and dotted curves are slightly different near equality (where the relative difference between the scale factors is noticeably large) and only for modes that entered the Hubble radius around or before equality. Moreover, the g.w. amplitudes of the modes that entered the Hubble radius before equality gradually equalize in course of later evolution. There is nothing like a huge underestimation or overestimation of the high-frequency g.w. power that was alleged to happen in the joined model.

It is easy to understand these features. Let us start from waves that entered the Hubble radius well after equality, i.e. waves with wave numbers $n / n_{H} \ll \sqrt{1+z_{\text {eq }}}$. As long as these waves are outside the Hubble radius, their amplitudes remain constant and equal in the two models, despite the fact that the scale factors are somewhat different near equality. The waves start oscillating in the regime where the relative difference between (27) and (28) is small, and therefore the mode functions, as functions of $\eta$, coincide.

The waves with wave numbers $n / n_{H} \gg \sqrt{1+z_{\mathrm{eq}}}$ enter the Hubble radius well before the equality. They oscillate in the WKB regime according to the law $h_{n}(\eta) \propto$ $e^{-i n \eta} / a(\eta)$, having started with equal amplitudes in the two models. Near equality, the mode functions are different in the two models, as much as the scale factors are different. But the relative difference between (27) and (28) decreases with time, and therefore the mode functions in the two models gradually equalize. The amplitudes of these mode functions, as well as the scale factors (27) and (28), are exactly equal today. The only difference between these high-frequency mode functions is in phase, that is, in different numbers of cycles that they experienced by today. This is because the moment of time defined as "today" in the two models is given, in terms of the common parameter $\eta$, by slightly different values of $\eta_{R}$.

Finally, for intermediate wave numbers $n / n_{H} \approx$ $\sqrt{1+z_{\mathrm{eq}}}$, the modes enter the Hubble radius when the scale factors differ the most. Therefore, they start oscillating with somewhat different amplitudes. The difference between these mode functions is noticeable by the redshift of decoupling $z_{\text {dec }}$ (characterized by somewhat different values of $\eta_{\text {dec }}$ ), as shown in Fig. 1. The difference survives until today, making the graph for the g.w. power spectrum (13) a little smoother (in comparison with that derived from the joined model) in the region of frequencies $10^{-16} \mathrm{~Hz}$ that correspond to the era of equality.
Having demonstrated that the use of joined analytical solutions is well justified, and the previously plotted graphs for $h_{\mathrm{rms}}(\nu)$ and $\Omega_{g w}(\nu)$ are essentially correct, we shall now exhibit the more accurate graphs based on numerical calculations with the initial conditions (29) and (30). We adopt $z_{\mathrm{eq}}=3 \times 10^{3}$ and $H_{0}=75 \frac{\mathrm{km}}{\mathrm{s}} / \mathrm{Mpc}$ [18]. We adjust the remaining free constant $z_{i}$ in such a way that the temperature correlation function $\ell(\ell+1) C_{\ell} / 2 \pi$ at $\ell=2$ is equal to $211 \mu \mathrm{K}^{2}$ [19]. This requires us to take $z_{i}=$ $1.0 \times 10^{29}$ for $\beta=-2$ and $z_{i}=2.4 \times 10^{30}$ for $\beta=$ -1.9 .

The graphs for today's spectra, normalized as stated above, are shown in Fig. 2. In order to keep the figure uncluttered, the oscillations of $h_{\mathrm{rms}}(\nu)$ are shown only at low frequencies, while the oscillations of $\Omega_{g w}(\nu)$ are not shown at all. We have also indicated possible detection techniques in various frequency bands.

The function $\Omega_{g w}(\nu)$ is the spectral value of the cosmological parameter $\Omega_{g w}[17,20]$ :

$$
\begin{aligned}
\Omega_{g w}\left(\nu_{1}, \nu_{2}\right) & =\frac{\rho_{g w}\left(\nu_{1}, \nu_{2}\right)}{\rho_{c}}=\frac{1}{\rho_{c}} \int_{\nu_{1}}^{\nu_{2}} \rho_{g w}(\nu) \frac{d \nu}{\nu} \\
& =\int_{\nu_{1}}^{\nu_{2}} \Omega_{g w}(\nu) \frac{d \nu}{\nu}
\end{aligned}
$$

that is,

$$
\Omega_{g w}(\nu)=\frac{1}{\rho_{c}} \rho_{g w}(\nu)
$$

Using the high-frequency definition of $\rho_{g w}(\nu)$ (valid only for waves which are significantly shorter than $l_{H}$ ) we derive [17]:

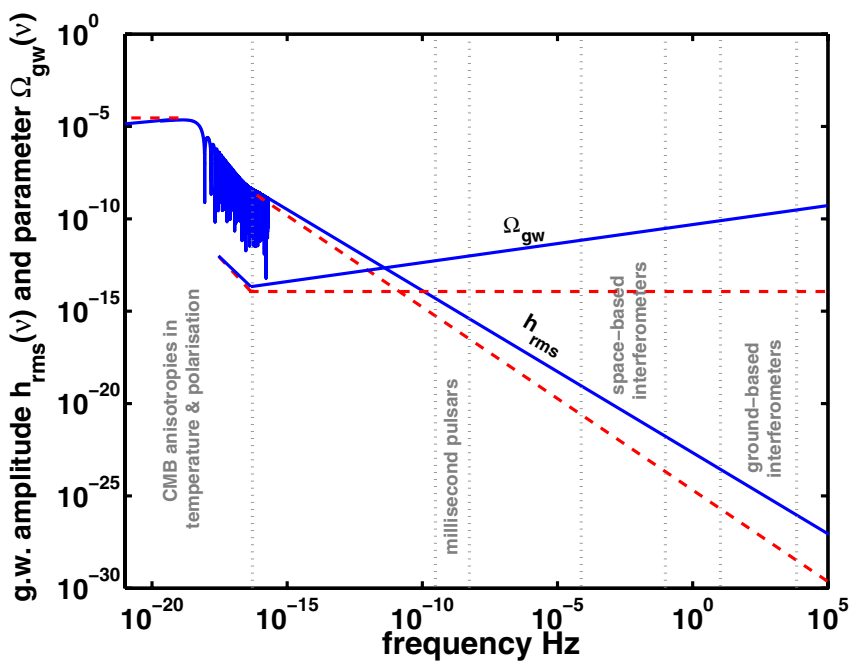

FIG. 2 (color online). The present-day spectra for $h_{\mathrm{rms}}(\nu)$ and $\Omega_{g w}(\nu)$. The solid lines correspond to the primordial spectral index $\beta=-1.9$, i.e. $\mathrm{n}=1.2$, while the dashed lines are for $\beta=$ -2 , i.e. $\mathrm{n}=1$. 


$$
\Omega_{g w}(\nu)=\frac{\pi^{2}}{3} h_{\mathrm{rms}}^{2}(\nu)\left(\frac{\nu}{\nu_{H}}\right)^{2} .
$$

It is this definition of $\Omega_{g w}(\nu)$ that is used for drawing the curves in Fig. 2.

We have to warn the reader that a great deal of literature on stochastic g.w. backgrounds uses the incorrect definition

$$
\Omega_{g w}(\nu)=\frac{1}{\rho_{c}} \frac{d \rho_{g w}(\nu)}{d \ln \nu},
$$

which suggests that the $\Omega_{g w}(\nu)$ parameter is zero if the g.w. spectral energy density $\rho_{g w}(\nu)$ is frequencyindependent, regardless of the numerical value of $\rho_{g w}(\nu)$. Then, from this incorrect definition, a formula similar to Eq. (32) is often being derived by making further compensating errors.

The higher-frequency part of $h_{\mathrm{rms}}(\nu)$ is relevant for direct searches for relic gravitational waves, while the lower-frequency part is relevant to the CMB calculations that we turn to in the next section. The direct and indirect methods of detecting relic gravitational waves are considered in a large number of papers (see, for example, $[5,20-$ 28] even though we disagree with some of them).

It is important to keep in mind that according to the inflationary theory the dashed lines for $\Omega_{g w}(\nu)$ and $h_{r m s}(\nu)$ in Fig. 2 should be at a zero level, because they describe the g.w. background with a flat primordial spectrum $\beta=-2$, $\mathrm{n}=1$. The "consistency relation" of the inflationary theory demands $r=0$, i.e. vanishingly small g.w. background, in the limit $\mathrm{n}=1$.

\section{EQUATIONS OF RADIATIVE TRANSFER}

\section{A. Characterization of a radiation field}

The radiation field is usually characterized by the four Stokes parameters $(I, Q, U, V),[29,30]$. The parameter $I$ is the total intensity of radiation, $Q$ and $U$ describe the magnitude and direction of linear polarization, and $V$ is the circular polarization. The Stokes parameters can be viewed as functions of photons' coordinates and momenta $\left(x^{\alpha}, p^{\alpha}\right)$. Since photons propagate with the speed of light $c$, the momenta satisfy the condition $p_{\alpha} p^{\alpha}=0$. Thus, the Stokes parameters are functions of $\left(t, x^{i}, \nu, e^{i}\right)$, where $\nu$ is the photon's frequency, and $e^{i}$ is a unit vector in the direction of observation (opposite to the photon's propagation).

In a given space-time point $\left(t, x^{i}\right)$, the Stokes parameters are functions of $\nu, \theta, \phi$, where $\theta, \phi$ are coordinates on a unit sphere:

$$
d \sigma^{2}=g_{a b} d x^{a} d x^{b}=d \theta^{2}+\sin ^{2} \theta d \phi^{2} .
$$

The radial direction is the direction of observation.

The Stokes parameters are components of the polarization tensor $P_{a b}$ [30] which can be written as

$$
P_{a b}(\theta, \phi)=\frac{1}{2}\left(\begin{array}{cc}
I+Q & -(U-i V) \sin \theta \\
-(U+i V) \sin \theta & (I-Q) \sin ^{2} \theta
\end{array}\right) .
$$

(We do not indicate the dependence of Stokes parameters on $\nu$.)

The symmetry of $P_{a b}$ with respect to rotations around the direction of observation requires the vanishing of linear polarization $Q=0, U=0$, but the circular polarization $V$ can be present. The symmetry of $P_{a b}$ with respect to coordinate reflections in the observation plane requires $V=0$, but the linear polarization can be present. The first symmetry means that the readings of a linear polarimeter are the same when it is rotated in the observation plane. The second symmetry means that the readings of the lefthanded and right-handed circular polarimeters are the same. (Compare with the discussion on gravitational waves in Sec. II A.)

Under arbitrary transformations of $\theta, \phi$, the components of $P_{a b}(\theta, \phi)$ transform as components of a tensor, but some quantities remain invariant. We want to build linear invariants from $P_{a b}$ and its derivatives, using the metric tensor $g_{a b}(\theta, \phi)$ and a completely antisymmetric pseudotensor $\epsilon^{a b}(\theta, \phi)$,

$$
\epsilon^{a b}=\left(\begin{array}{cc}
0 & -\sin ^{-1} \theta \\
\sin ^{-1} \theta & 0
\end{array}\right)
$$

The first two invariants are easy to build:

$$
\begin{aligned}
I(\theta, \phi) & =g^{a b}(\theta, \phi) P_{a b}(\theta, \phi), \\
V(\theta, \phi) & =i \epsilon^{a b}(\theta, \phi) P_{a b}(\theta, \phi) .
\end{aligned}
$$

Then, it is convenient to single out the trace and antisymmetric parts of $P_{a b}$, and introduce the symmetric trace-free (STF) part $P_{a b}^{\mathrm{STF}}$ :

$$
\begin{aligned}
P_{a b}(\theta, \phi) & =\frac{1}{2} I g_{a b}-\frac{i}{2} V \epsilon_{a b}+P_{a b}^{\mathrm{STF}}, \\
P_{a b}^{\mathrm{STF}} & =\frac{1}{2}\left(\begin{array}{cc}
Q & -U \sin \theta \\
-U \sin \theta & -Q \sin ^{2} \theta
\end{array}\right) .
\end{aligned}
$$

Clearly, the construction of other linear invariants requires the use of covariant derivatives of the tensor $P_{a b}^{\mathrm{STF}}$. There is no invariants that can be built from the first derivatives $P_{a b ; c}^{\mathrm{STF}}$, so we need to go to the second derivatives. One can check that there are only two linearly independent invariants that can be built from the second derivatives:

$$
E(\theta, \phi)=-2\left(P_{a b}^{\mathrm{STF}}\right) ; a ; b, \quad B(\theta, \phi)=-2\left(P_{a b}^{\mathrm{STF}}\right) ; b ; d \epsilon_{d}^{a},
$$

The quantities $I$ and $E$ are scalars, while $V$ and $B$ are pseudoscalars. $V$ and $B$ change sign under flips of directions (coordinate transformations with negative determinants). This is also seen from the fact that their construction involves the pseudotensor $\epsilon_{a b}$. 
The invariant quantities $(I, V, E, B)$, as functions of $\theta, \phi$, can be expanded over ordinary spherical harmonics $Y_{\ell m}(\theta, \phi), Y_{\ell m}^{*}=(-1)^{m} Y_{\ell,-m}$ :

$$
\begin{aligned}
I(\theta, \phi) & =\sum_{\ell=0}^{\infty} \sum_{m=-\ell}^{\ell} a_{\ell m}^{T} Y_{\ell m}(\theta, \phi), \\
V(\theta, \phi) & =\sum_{\ell=0}^{\infty} \sum_{m=-\ell}^{\ell} a_{\ell m}^{V} Y_{\ell m}(\theta, \phi), \\
E(\theta, \phi) & =\sum_{\ell=2}^{\infty} \sum_{m=-\ell}^{\ell}\left[\frac{(\ell+2) !}{(\ell-2) !}\right]^{1 / 2} a_{\ell m}^{E} Y_{\ell m}(\theta, \phi), \\
B(\theta, \phi) & =\sum_{\ell=2}^{\infty} \sum_{m=-\ell}^{\ell}\left[\frac{(\ell+2) !}{(\ell-2) !}\right]^{1 / 2} a_{\ell m}^{B} Y_{\ell m}(\theta, \phi) .
\end{aligned}
$$

The set of multipole coefficients $\left(a_{\ell m}^{T}, a_{\ell m}^{V}, a_{\ell m}^{E}, a_{\ell m}^{B}\right)$ completely characterizes the radiation field. We will use these quantities in our further discussion.

To make contact with previous work, we note that the multipole coefficients $a_{\ell m}^{E}, a_{\ell m}^{B}$ can also be expressed in terms of the tensor $P_{a b}$ itself, rather than its derivatives. This is possible because one can interchange the order of differentiation under the integrals that define $a_{\ell m}^{E}, a_{\ell m}^{B}$ in terms of the right-hand side (r.h.s.) of Eq. (36). This leads to the appearance of the spin-weighted spherical harmonics or tensor spherical harmonics [12-14,31,32]. For example, the tensor $P_{a b}$ can be written as

$$
\begin{aligned}
P_{a b}= & \frac{1}{2} \sum_{\ell=0}^{\infty} \sum_{m=-\ell}^{\ell}\left(g_{a b} a_{\ell m}^{T}-i \epsilon_{a b} a_{\ell m}^{V}\right) Y_{\ell m}(\theta, \phi) \\
& +\frac{1}{\sqrt{2}} \sum_{\ell=2}^{\infty} \sum_{m=-l}^{l}\left(-a_{\ell m}^{E} Y_{(\ell m) a b}^{G}(\theta, \phi)\right. \\
& \left.+a_{\ell m}^{B} Y_{(\ell m) a b}^{C}(\theta, \phi)\right),
\end{aligned}
$$

where $Y_{(\ell m) a b}^{G}(\theta, \phi)$ and $Y_{(\ell m) a b}^{C}(\theta, \phi)$ are the "gradient" and "curl" tensor spherical harmonics forming a set of orthonormal functions for STF tensors [32]. The invariants $E$ and $B$ can also be written in terms of the spin raising and lowering operators $ð$ and $\bar{\partial}[31]$ :

$$
\begin{aligned}
& E=-\frac{1}{2}\left[\bar{ð}^{2}(Q+i U)+ð^{2}(Q-i U)\right], \\
& B=\frac{i}{2}\left[\bar{ð}^{2}(Q+i U)-ð^{2}(Q-i U)\right] .
\end{aligned}
$$

The quantities $E$ and $B$ are called the $E$ (or gradient) and $B$ (or curl) modes of polarization. The $\ell$-dependent numerical coefficients in (37c) and (37d) were introduced in order to make the definitions of this paper fully consistent with the previous literature [31,32].

\section{B. Radiative transfer in a perturbed universe}

We need to work out the radiative transfer equation in a slightly perturbed universe described by Eq. (1). The
Thompson scattering of initially unpolarized light cannot generate circular polarization, so we shall not consider the $V$ Stokes parameter. Following [29,33,34], we shall write the radiative transfer equation in terms of a 3-component quantity (symbolic vector) $\hat{\mathbf{n}}\left(x^{\alpha}, p^{\alpha}\right)$. The components $\left(\hat{n}_{1}, \hat{n}_{2}, \hat{n}_{3}\right)$ are related to the Stokes parameters by

$$
\hat{\mathbf{n}}=\left(\begin{array}{l}
\hat{n}_{1} \\
\hat{n}_{2} \\
\hat{n}_{3}
\end{array}\right)=\frac{1}{2} \frac{c^{2}}{h \nu^{3}}\left(\begin{array}{c}
I+Q \\
I-Q \\
-2 U
\end{array}\right),
$$

where $h$ is the Planck constant. The quantities $\hat{n}_{1}, \hat{n}_{2},\left(\hat{n}_{1}+\right.$ $\left.\hat{n}_{2}+\hat{n}_{3}\right) / 2$ are the numbers of photons of frequency $\nu$ coming from the direction $z$ and passing through a slit oriented, respectively, in the directions $x, y$, and the bisecting direction between $x$ and $y$.

The equation of radiative transfer can be treated as a Boltzmann equation in a phase space. The general form of this equation is as follows [35]

$$
\frac{D \hat{\mathbf{n}}}{d s}=\hat{\mathbf{C}}[\hat{\mathbf{n}}],
$$

where $s$ is a parameter along the worldline of a photon, $\frac{D}{d s}$ is a total derivative along this worldline, and $\hat{\mathbf{C}}$ is a collision term. We shall explain each term of this equation separately.

The total derivative in Eq. (39) reads:

$$
\frac{D \hat{\mathbf{n}}}{d s}=\left[\frac{d x^{\alpha}}{d s} \frac{\partial}{\partial x^{\alpha}}+\frac{d p^{\alpha}}{d s} \frac{\partial}{\partial p^{\alpha}}\right] \hat{\mathbf{n}},
$$

where $d x^{\alpha} / d s$ and $d p^{\alpha} / d s$ are determined by the lightlike geodesic worldline,

$$
\frac{d x^{\alpha}}{d s}=p^{\alpha}, \quad \frac{d p^{\alpha}}{d s}=-\Gamma_{\beta \gamma}^{\alpha} p^{\beta} p^{\gamma}, \quad g_{\alpha \beta} p^{\alpha} p^{\beta}=0 .
$$

Strictly speaking, the square bracket in Eq. (40) should also include the additive matrix term $\hat{\mathbf{R}}$. This term is responsible for the rotation of polarization axes that may take place in course of parallel transport along the photon's geodesic line [36]. In the perturbation theory that we are working with, this matrix does not enter the equations in the zeroth and first-order approximations [33], and therefore we neglect $\hat{\mathbf{R}}$.

In our problem, the collision term $\hat{\mathbf{C}}$ describes the Thompson scattering of light on free (not combined in atoms) electrons [29]. We assume that the electrons are at rest with respect to one of synchronous coordinate systems (1). We work with this coordinate system, so that it is not only synchronous but also "comoving" with the electrons. (This choice is always possible when the functions $h_{i j}$ in (1) are gravitational waves. Certain complications in the case of density perturbations will be considered later, Appendix D.) Thus, the collision term $\hat{\mathbf{C}}$ is given by the expression 


$$
\begin{aligned}
\hat{\mathbf{C}}[\hat{\mathbf{n}}]= & -\sigma_{T} N_{e}\left(x^{\alpha}\right)\left(\frac{c d t}{d s}\right)\left[\hat{\mathbf{n}}\left(t, x^{i}, \nu, \theta, \phi\right)-\frac{1}{4 \pi}\right. \\
& \left.\times \int d \Omega^{\prime} \hat{\mathbf{P}}\left(\theta, \phi ; \theta^{\prime}, \phi^{\prime}\right) \hat{\mathbf{n}}\left(t, x^{i}, \nu, \theta^{\prime}, \phi^{\prime}\right)\right],
\end{aligned}
$$

where $\sigma_{T}=6.65 \cdot 10^{-24} \mathrm{~cm}^{2}$ is the Thompson cross section, $N_{e}$ is the density of free electrons, and $\hat{\mathbf{P}}\left(\theta, \phi ; \theta^{\prime}, \phi^{\prime}\right)$ is the Chandrasekhar scattering matrix. (The explicit form of the scattering matrix is discussed in Appendix C.) The factor $c d t / d s$ arises because of our use of the element $d s$, instead of $c d t$, in the left-hand side (1.h.s.) of Eq. (39). In accord with the meaning of the scattering term, the quantity $\sigma_{T} N_{e}\left(x^{\alpha}\right)(c d t / d s)$ is the averaged number of electrons that could participate in the scattering process when the photon traversed the element $d s$ along its path.

Let us now write down the equations of radiative transfer in the presence of the gravitational field (1). First, we write down the equations for the lightlike geodesic line $x^{\alpha}(s)=$ $\left(\eta(s), x^{i}(s)\right)$ :

$$
\begin{gathered}
p^{0}=\frac{d \eta}{d s}=\frac{\nu}{c a}, \quad p^{i}=\frac{d x^{i}}{d s}=\frac{\nu}{c a} e^{i}, \\
\frac{d \nu}{d s}=-\nu\left[\frac{1}{a} \frac{d a}{d \eta}+\frac{1}{2} e^{i} e^{j} \frac{\partial h_{i j}}{\partial \eta}\right] \frac{d \eta}{d s} \\
\left(\delta_{i j}+h_{i j}\right) e^{i} e^{j}=1 .
\end{gathered}
$$

We do not need the expression for $d e^{i} / d s$, because it is a first-order (in terms of metric perturbations) quantity, and this quantity enters the equations of radiative transfer only in products with other first-order terms. We neglect such second-order corrections.

Second, we write for the "vector" $\hat{\mathbf{n}}$ :

$$
\hat{\mathbf{n}}=\hat{\mathbf{n}}^{(0)}+\hat{\mathbf{n}}^{(1)},
$$

where $\hat{\mathbf{n}}^{(0)}$ is the zeroth order solution, and $\hat{\mathbf{n}}^{(1)}$ is the firstorder correction arising because of the presence of metric perturbations. We shall now formulate the equation for $\hat{\mathbf{n}}^{(1)}$, taking into account the zero-order solution to Eq. (39).

In the zero-order approximation we assume that $h_{i j}=0$ and that the radiation field is fully homogeneous, isotropic, and unpolarized. Therefore,

$$
\hat{\mathbf{n}}^{(0)}=n^{(0)}(\eta, \nu) \hat{\mathbf{u}}
$$

where

$$
\hat{\mathbf{u}}=\left(\begin{array}{l}
1 \\
1 \\
0
\end{array}\right) \text {. }
$$

Since the scattering matrix $\hat{\mathbf{P}}$ does not couple to the radiation field if it has no quadrupole anisotropy, the collision term (42) vanishes, $\hat{\mathbf{C}}\left[\hat{\mathbf{n}}^{(0)}\right]=0$, and the equation for $n^{(0)}(\eta, \nu)$ reads

$$
\frac{\partial n^{(0)}}{\partial \eta}-\frac{\nu}{a} \frac{d a}{d \eta} \frac{\partial n^{(0)}}{\partial \nu}=0
$$

The general solution to this equation is $n^{(0)}=n_{0}(\nu a(\eta))$, which makes it convenient to use a new variable

$$
\tilde{\nu}=\nu a(\eta) .
$$

In the zero-order approximation, $\nu=\mathrm{const} / a(\eta)$ and therefore $\tilde{\nu}$ is a constant along the light ray.

We are now in a position to write down the first-order approximation to the Boltzmann Eq. (39). We take $\left(\eta, x^{i}, \tilde{\nu}, e^{i}\right)$ as independent variables, i.e. $n^{(0)}=n_{0}(\tilde{\nu})$, $\hat{\mathbf{n}}^{(1)}=\hat{\mathbf{n}}^{(1)}\left(\eta, x^{i}, \tilde{\nu}, e^{i}\right)$, and use the identity

$$
\frac{d p^{\alpha}}{d s} \frac{\partial}{\partial p^{\alpha}}=\frac{d \tilde{\nu}}{d s} \frac{\partial}{\partial \tilde{\nu}}+\frac{d e^{i}}{d s} \frac{\partial}{\partial e^{i}}
$$

in the first-order approximation to (40). Taking also into account the geodesic Eq. (43) we arrive at the equation

$$
\left[\frac{\partial \hat{\mathbf{n}}^{(1)}}{\partial \eta}+e^{i} \frac{\partial \hat{\mathbf{n}}^{(1)}}{\partial x^{i}}-\frac{1}{2} \tilde{\nu} e^{i} e^{j} \frac{\partial h_{i j}}{\partial \eta} \frac{\partial \hat{\mathbf{n}}^{(0)}}{\partial \tilde{\nu}}\right] \frac{d \eta}{d s}=\hat{\mathbf{C}}\left[\hat{\mathbf{n}}^{(1)}\right] .
$$

Introducing new notations $q(\eta)=\sigma_{T} a(\eta) N_{e}(\eta)$ and $f(\tilde{\nu})=\partial \ln n_{0} / \partial \ln \tilde{\nu}$ [33] (the astrophysical meaning and numerical values of the functions $q(\eta)$ and $f(\tilde{\nu})$ are discussed in Appendix B) we write down the final form of the transfer equation:

$$
\begin{gathered}
{\left[\frac{\partial}{\partial \eta}+q(\eta)+e^{i} \frac{\partial}{\partial x^{i}}\right] \hat{\mathbf{n}}^{(1)}\left(\eta, x^{i}, \tilde{\nu}, e^{i}\right)} \\
=\frac{f(\tilde{\nu}) n_{0}(\tilde{\nu})}{2} e^{i} e^{j} \frac{\partial h_{i j}}{\partial \eta} \hat{\mathbf{u}}+q(\eta) \frac{1}{4 \pi} \\
\quad \times \int d \Omega^{\prime} \hat{\mathbf{P}}\left(e^{i} ; e^{\prime j}\right) \hat{\mathbf{n}}^{(1)}\left(\eta, x^{i}, \tilde{\nu}, e^{\prime j}\right) .
\end{gathered}
$$

It is seen from Eq. (46) that the "source" for the generation of $\hat{\mathbf{n}}^{(1)}$ consists of two terms on the r.h.s. of this equation. First, it is the gravitational-field perturbation $h_{i j}$, participating in the combination $e^{i} e^{j} \partial h_{i j} / \partial \eta$. It directly generates a structure proportional to $\hat{\mathbf{u}}$, i.e. a variation in the $I$ Stokes parameter and a temperature anisotropy. In this process, a quadrupole component of the temperature anisotropy necessarily arises due to the presence of the term $e^{i} \partial / \partial x^{i}$, even if the above-mentioned combination itself does not have angular dependence. The second term on the r.h.s. of Eq. (46) generates polarization, i.e. a structure different from $\hat{\mathbf{u}}$. This happens because of the mixing of different components of $\hat{\mathbf{n}}^{(1)}$, including those proportional to $\hat{\mathbf{u}}$, in the product term $\hat{\mathbf{P}} \hat{\mathbf{n}}^{(1)}$. In other words, polarization is generated by the scattering of anisotropic radiation field. Clearly, polarization is generated only in the intervals of time when $q(\eta) \neq 0$, i.e. when free electrons are available for the Thompson scattering (see, for example, [37]). 


\section{The radiative transfer equations for a single gravitational wave}

We work with a random gravitational field $h_{i j}$ expanded over spatial Fourier components (10). It is convenient to make similar expansion for the quantities $\hat{\mathbf{n}}^{(1)}\left(\eta, x^{i}, \tilde{\nu}, e^{i}\right)$. Since Eq. (46) is linear, the Fourier components of $\hat{\mathbf{n}}^{(1)}$ inherit the same random coefficients $\stackrel{s}{c_{\mathbf{n}}}$ that enter Eq. (10):

$$
\hat{\mathbf{n}}^{(1)}\left(\eta, x^{i}, \tilde{\nu}, e^{i}\right)=\frac{\mathcal{C}}{(2 \pi)^{3 / 2}} \int_{-\infty}^{+\infty} \frac{d^{3} \mathbf{n}}{\sqrt{2 n}} \sum_{s=1,2}\left[\hat{\mathbf{n}}_{\mathbf{n}, s}^{(1)}\left(\eta, \tilde{\nu}, e^{i}\right) e^{i \mathbf{n} \cdot \mathbf{x}^{s}}{ }_{\mathbf{n}_{\mathbf{n}}}+\hat{\mathbf{n}}_{\mathbf{n}, s}^{(1) *}\left(\eta, \tilde{\nu}, e^{i}\right) e^{-i \mathbf{n} \cdot \mathbf{x}^{s^{*}}}{ }_{\mathbf{n}}\right]
$$

Equation (46) for a particular Fourier component takes the form:

$$
\left[\frac{\partial}{\partial \eta}+q(\eta)+i e^{i} n_{i}\right] \hat{\mathbf{n}}_{\mathbf{n}, s}^{(1)}\left(\eta, \tilde{\nu}, e^{i}\right)=\frac{f(\tilde{\nu}) n_{0}(\tilde{\nu})}{2} e^{i} e^{j} \stackrel{p}{p}_{i j}(\mathbf{n}) \frac{d h_{n}(\eta)}{d \eta} \hat{\mathbf{u}}+\frac{q(\eta)}{4 \pi} \int d \Omega^{\prime} \hat{\mathbf{P}}\left(e^{i} ; e^{\prime j}\right) \hat{\mathbf{n}}_{\mathbf{n}, s}^{(1)}\left(\eta, \tilde{\nu}, e^{\prime j}\right) .
$$

To simplify technical details, we start with a single gravitational wave propagating exactly in the direction of $z$ in terms of definitions (2), (4), and (19). The coordinate system, associated with the wave, is specified by $\theta=0$, $\phi=0$. This simplifies the polarization tensors (19) and makes them constant matrices. At the same time, the observation direction is arbitrary and is defined by $e^{i}=$ $(\sin \theta \cos \phi, \sin \theta \sin \phi, \cos \theta)$. We consider circularly polarized states with $s=1=L, s=2=R$. Then, we find

$$
e^{i} e^{j}{\stackrel{s}{p_{i j}}}_{(\mathbf{n})}=\left(1-\mu^{2}\right) e^{ \pm 2 i \phi},
$$

where $\mu=\cos \theta$ and the \pm signs correspond to $s=L, R$, respectively. This simplification of the angular dependence is possible only for one Fourier component, but not for all of them together. We shall still need the results for a wave propagating in an arbitrary direction. The necessary generalization will be done in Sec. V B.

The $\pm 2 \phi$ angular dependence of the source term in Eq. (48) generates the $\pm 2 \phi$ angular dependence in the solution $[33,34]$. We show in Appendix $C$ that the terms in $\hat{\mathbf{n}}_{\mathbf{n}, s}^{(1)}(\eta, \tilde{\nu}, \mu, \phi)$ with any other $\phi$-dependence satisfy homogeneous differential equations, and therefore they vanish if they were not present initially (which we always assume). Similarly, the $\tilde{\nu}$ dependence of the solution can be factored out. Finally, we show in Appendix $\mathrm{C}$ that one linear combination of the three components of $\hat{\mathbf{n}}_{\mathbf{n}}^{(1)}$ always satisfies a homogeneous equation and therefore vanishes at zero initial data. Thus, the problem of solving Eq. (48) reduces to the problem of finding two functions of the arguments $\eta, \mu$.

Explicitly, we can now write

$$
\begin{aligned}
\hat{\mathbf{n}}_{n, s}^{(1)}(\eta, \tilde{\nu}, \mu, \phi)= & \frac{f(\tilde{\nu}) n_{0}(\tilde{\nu})}{2}\left[\alpha_{n, s}(\eta, \mu)\left(1-\mu^{2}\right)\left(\begin{array}{l}
1 \\
1 \\
0
\end{array}\right)\right. \\
& \left.+\beta_{n, s}(\eta, \mu)\left(\begin{array}{c}
\left(1+\mu^{2}\right) \\
-\left(1+\mu^{2}\right) \\
\mp 4 i \mu
\end{array}\right)\right] e^{ \pm 2 i \phi} .
\end{aligned}
$$

Clearly, function $\alpha$ is responsible for temperature anisotropy (I Stokes parameter), while function $\beta$ is responsible for polarization ( $Q$ and $U$ Stokes parameters).

Temporarily dropping out the labels $n, s$ and introducing the auxiliary function $\xi(\eta, \mu)=\alpha(\eta, \mu)+\beta(\eta, \mu)$, we get from Eqs. (48) and (50) a pair of coupled equations [34]:

$$
\frac{\partial \beta(\eta, \mu)}{\partial \eta}+(q(\eta)+i n \mu) \beta(\eta, \mu)=\frac{3}{16} q(\eta) I(\eta),
$$

$$
\frac{\partial \xi(\eta, \mu)}{\partial \eta}+(q(\eta)+i n \mu) \xi(\eta, \mu)=\frac{d h(\eta)}{d \eta}
$$

where

$$
\begin{aligned}
I(\eta)= & \int_{-1}^{1} d \mu^{\prime}\left[\left(1+\mu^{\prime 2}\right)^{2} \beta\left(\eta, \mu^{\prime}\right)\right. \\
& \left.-\frac{1}{2}\left(1-\mu^{\prime 2}\right)^{2} \xi\left(\eta, \mu^{\prime}\right)\right] .
\end{aligned}
$$

\section{RADIATIVE TRANSFER EQUATIONS AS A SINGLE INTEGRAL EQUATION}

In some previous studies [31,38,39], Eqs. (51) and (52) are being solved by first expanding the $\mu$-dependence in terms of Legendre polynomials. This generates an infinite series of coupled ordinary differential equations. Then, the series is being truncated at some order.

We go by a different road. We demonstrate that the problem can be reduced to a single mathematically consistent integral equation. There are technical and interpretational advantages in this approach. The integral equation enables us to derive physically transparent analytical solutions and make reliable estimates of the generated polarization. The numerical implementation of the integral equation considerably saves computing time and allows simple control of accuracy. 


\section{A. Derivation of the integral equation}

In order to show that the solutions of Eqs. (51) and (52) for $\alpha(\eta, \mu)$ and $\beta(\eta, \mu)$ are completely determined by a single integral equation, we first introduce new quantities [40]

$$
\begin{aligned}
& \Phi(\eta)=\frac{3}{16} g(\eta) I(\eta), \\
& H(\eta)=e^{-\tau(\eta)} \frac{d h(\eta)}{d \eta} .
\end{aligned}
$$

Solutions to Eqs. (51) and (52) can be written as

$$
\begin{aligned}
& \beta(\eta, \mu)=e^{\tau(\eta)-i n \mu \eta} \int_{0}^{\eta} d \eta^{\prime} \Phi\left(\eta^{\prime}\right) e^{i n \mu \eta^{\prime}}, \\
& \xi(\eta, \mu)=e^{\tau(\eta)-i n \mu \eta} \int_{0}^{\eta} d \eta^{\prime} H\left(\eta^{\prime}\right) e^{i n \mu \eta^{\prime}},
\end{aligned}
$$

Expression (56) is a formal solution to Eq. (51) in the sense that $\beta(\eta, \mu)$ is expressed in terms of $\Phi(\eta)$ which itself depends on $\beta(\eta, \mu)$ (see (53) and (54)).

We now put (56) and (57) into Eq. (53) to get a new formulation for $I(\eta)$ :

$$
\begin{aligned}
I(\eta)= & e^{\tau(\eta)} \int_{-1}^{1} \int_{0}^{\eta} d \mu d \eta^{\prime}\left[\left(1+\mu^{2}\right)^{2} \Phi\left(\eta^{\prime}\right)\right. \\
& \left.-\frac{1}{2}\left(1-\mu^{2}\right)^{2} H\left(\eta^{\prime}\right)\right] e^{i n \mu\left(\eta^{\prime}-\eta\right)} .
\end{aligned}
$$

Using the kernels $K_{ \pm}\left(\eta-\eta^{\prime}\right)$,

$$
K_{ \pm}\left(\eta-\eta^{\prime}\right)=\int_{-1}^{1} d \mu\left(1 \pm \mu^{2}\right)^{2} e^{i n \mu\left(\eta-\eta^{\prime}\right)},
$$

Equation (58) can be rewritten as

$$
\begin{aligned}
I(\eta)= & e^{\tau(\eta)} \int_{0}^{\eta} d \eta^{\prime}\left[K_{+}\left(\eta-\eta^{\prime}\right) \Phi\left(\eta^{\prime}\right)\right. \\
& \left.-\frac{1}{2} K_{-}\left(\eta-\eta^{\prime}\right) H\left(\eta^{\prime}\right)\right] .
\end{aligned}
$$

Multiplying both sides of this equality by $(3 / 16) q(\eta) e^{-\tau(\eta)}$ and recalling the definition (54) we arrive at a closed form equation for $\Phi(\eta)$ :

$$
\Phi(\eta)=\frac{3}{16} q(\eta) \int_{0}^{\eta} d \eta^{\prime} \Phi\left(\eta^{\prime}\right) K_{+}\left(\eta-\eta^{\prime}\right)+F(\eta),
$$

where $F(\eta)$ is the known gravitational-field term given by the metric perturbations,

$$
F(\eta)=-\frac{3}{32} q(\eta) \int_{0}^{\eta} d \eta^{\prime} H\left(\eta^{\prime}\right) K_{-}\left(\eta-\eta^{\prime}\right),
$$

The derived Eq. (61) for $\Phi(\eta)$ is the integral equation of Voltairre type. As soon as $\Phi(\eta)$ is found from this equation, we can find $\beta(\eta, \mu)$ from Eq. (56). Then, Eqs. (56) and (57) completely determine all the components of $\hat{\mathbf{n}}^{(1)}$ according to Eq. (50).

Clearly, we are mainly interested in temperature and polarization anisotropies seen at the present time $\eta=$ $\eta_{R}$. Introducing $\zeta=n\left(\eta_{R}-\eta\right)$ and restoring the indices $n$ and $s$, we obtain the present-day values of $\alpha$ and $\beta$ :

$\alpha_{n, s}(\mu) \equiv \alpha_{n, s}\left(\eta_{R}, \mu\right)=\int_{0}^{\eta_{R}} d \eta\left(H_{n, s}(\eta)-\Phi_{n, s}(\eta)\right) e^{-i \mu \zeta}$,

$\beta_{n, s}(\mu) \equiv \beta_{n, s}\left(\eta_{R}, \mu\right)=\int_{0}^{\eta_{R}} d \eta \Phi_{n, s}(\eta) e^{-i \mu \zeta}$

The integrals can safely be taken from $\eta=0$ as the optical depth $\tau$ quickly becomes very large in the early Universe, and the source functions $H_{n, s}(\eta)$ and $\Phi_{n, s}(\eta)$ quickly vanish there. We will work with expressions (63) in our further calculations.

\section{B. Analytical solution to the integral equation}

The integral Eq. (61) can be solved analytically in the form of a series expansion. Although our graphs and physical conclusions in this paper are based on the exact numerical solution to Eq. (61), it is important to have a simple analytical approximation to the exact numerical solution. We will show below why the infinite series can be accurately approximated by its first term and how this simplification helps in physical understanding of the derived numerical results.

We start with the transformation of kernels (59) of the integral Eq. (61). Using the identity $\mu^{k} e^{i x \mu}=$ $(d / i d x)^{k} e^{i x \mu}$, the kernels can be written as

$$
\begin{aligned}
K_{ \pm}\left(\eta-\eta^{\prime}\right) & =\int_{-1}^{+1} d \mu\left(1 \pm \mu^{2}\right)^{2} e^{i n \mu\left(\eta-\eta^{\prime}\right)} \\
& =2\left(1 \mp \frac{d^{2}}{d x^{2}}\right)^{2} \frac{\sin x}{x}
\end{aligned}
$$

where $x=n\left(\eta-\eta^{\prime}\right)$. Now, taking into account the expansion

$$
\frac{\sin x}{x}=\sum_{m=0}^{\infty} \frac{(-1)^{m}}{(2 m+1) !} x^{2 m}
$$

the r.h.s. of Eq. (61) can be presented in the form of a series:

$$
\begin{aligned}
\Phi(\eta)= & \frac{3}{2} q(\eta) \sum_{m=0}^{\infty} n^{2 m} \int_{0}^{\eta} d \eta^{\prime}\left(\eta-\eta^{\prime}\right)^{2 m} \\
& \times\left[\lambda_{+}(m) \Phi\left(\eta^{\prime}\right)-\lambda_{-}(m) H\left(\eta^{\prime}\right)\right]
\end{aligned}
$$

where 


$$
\begin{aligned}
& \lambda_{+}(m)=\frac{(-1)^{m}}{(2 m+1) !}\left[1-4 \frac{(m+2)}{(2 m+3)(2 m+5)}\right] \\
& \lambda_{-}(m)=\frac{(-1)^{m}}{(2 m+1) !(2 m+3)(2 m+5)} .
\end{aligned}
$$

Since the r.h.s. of Eq. (64) is a series in even powers of the wave number $n$, the l.h.s. of the same equation can also be expanded in powers of $n^{2 m}$ :

$$
\Phi(\eta)=\sum_{m=0}^{\infty} \Phi^{(m)}(\eta) n^{2 m}
$$

Using expansion (65) in both sides of Eq. (64) we transform this equation to

$$
\sum_{m=0}^{\infty} \Phi^{(m)}(\eta) n^{2 m}=\frac{3}{2} q(\eta)\left[\sum_{m=0}^{\infty} \sum_{j=0}^{\infty} \lambda_{+}(m) n^{2(m+j)} \int_{0}^{\eta} d \eta^{\prime} \Phi^{(j)}\left(\eta^{\prime}\right)\left(\eta-\eta^{\prime}\right)^{2 m}-\sum_{m=0}^{\infty} \lambda_{-}(m) n^{2 m} \int_{0}^{\eta} d \eta^{\prime} H\left(\eta^{\prime}\right)\left(\eta-\eta^{\prime}\right)^{2 m}\right]
$$

The left side and the second term in the right side of Eq. (66) are series in $n^{2 m}$, but the first sum on the r.h.s. of Eq. (66) is still a mixture of different powers. This sum can be rearranged to be manifestly a series in $n^{2 m}$ :

$$
\sum_{m=0}^{\infty} \sum_{j=0}^{\infty} \lambda_{+}(m) n^{2(m+j)} \int_{0}^{\eta} d \eta^{\prime} \Phi^{(j)}\left(\eta^{\prime}\right)\left(\eta-\eta^{\prime}\right)^{2 m}=\sum_{m=0}^{\infty} n^{2 m}\left[\sum_{k=0}^{m} \lambda_{+}(k) \int_{0}^{\eta} d \eta^{\prime} \Phi^{(m-k)}\left(\eta^{\prime}\right)\left(\eta-\eta^{\prime}\right)^{2 k}\right]
$$

According to Eq. (66) we have to make equal the coefficients of terms with the same power $n^{2 m}$ in both sides of the equation. This produces a set of integral equations

$$
\Phi^{(m)}(\eta)=q(\eta) S^{(m)}(\eta)+\frac{7}{10} q(\eta) \int_{0}^{\eta} d \eta^{\prime} \Phi^{(m)}\left(\eta^{\prime}\right)
$$

where functions $S^{(m)}(\eta)$ depend only on the known function $H(\eta)$ and functions $\Phi^{(m-k)}(\eta)$ presumed to be found from equations of previous orders:

$$
\begin{aligned}
S^{(m)}(\eta)= & -\frac{3}{2} \lambda_{-}(m) \int_{0}^{\eta} d \eta^{\prime} H\left(\eta^{\prime}\right)\left(\eta-\eta^{\prime}\right)^{2 m} \\
& +\frac{3}{2} \sum_{k=1}^{m} \lambda_{+}(k) \int_{0}^{\eta} d \eta^{\prime} \Phi^{(m-k)}\left(\eta^{\prime}\right)\left(\eta-\eta^{\prime}\right)^{2 k}
\end{aligned}
$$

The important advantage of the performed expansion in powers of $n^{2 m}$ is that Eq. (68) of any order $m$ is now a selfcontained analytically solvable integral equation.

Exact solution to the integral Eq. (68) is given by the formula

$$
\Phi^{(m)}(\eta)=q(\eta) \int_{0}^{\eta} d \eta^{\prime} \frac{d S^{(m)}\left(\eta^{\prime}\right)}{d \eta^{\prime}} e^{(7 / 10) \tau\left(\eta, \eta^{\prime}\right)}
$$

We can further simplify this formula. Taking an $\eta$-derivative of expression (69) we find

$$
\begin{aligned}
\frac{d S^{(m)}(\eta)}{d \eta}= & -\frac{1}{10} H(\eta) \delta_{0 m}-\omega_{-}(m) \\
& \times \int_{0}^{\eta} d \eta^{\prime} H\left(\eta^{\prime}\right)\left(\eta-\eta^{\prime}\right)^{2 m-1} \\
& +\sum_{k=1}^{m} \omega_{+}(k) \int_{0}^{\eta} d \eta^{\prime} \Phi^{(m-k)}\left(\eta^{\prime}\right)\left(\eta-\eta^{\prime}\right)^{2 k-1},
\end{aligned}
$$

where $\omega_{ \pm}(m)=3 m \lambda_{ \pm}(k)$. Substituting this expression into Eq. (70), we arrive at the final result

$$
\begin{aligned}
\Phi^{(m)}(\eta)= & q(\eta) e^{-(7 / 10) \tau(\eta)} \int_{0}^{\eta} d \eta^{\prime} e^{(7 / 10) \tau\left(\eta^{\prime}\right)} \\
& \times\left[-H\left(\eta^{\prime}\right)\left(\frac{1}{10} \delta_{0 m}+\omega_{-}(m) \Psi_{(m)}\left(\eta, \eta^{\prime}\right)\right)\right. \\
& \left.+\sum_{k=1}^{m} \omega_{+}(k) \Phi^{(m-k)}\left(\eta^{\prime}\right) \Psi_{(k)}\left(\eta, \eta^{\prime}\right)\right],
\end{aligned}
$$

where

$$
\Psi_{(k)}\left(\eta, \eta^{\prime}\right)=\int_{\eta^{\prime}}^{\eta} d \eta^{\prime \prime} e^{-(7 / 10) \tau\left(\eta^{\prime \prime}, \eta^{\prime}\right)}\left(\eta^{\prime \prime}-\eta^{\prime}\right)^{2 k-1}
$$

Functions $\Psi_{(k)}\left(\eta, \eta^{\prime}\right)$ depend only on the ionization history of the background cosmological model described by $q(\eta)$. These functions can be computed in advance. Complete determination of the function $\Phi(\eta)$, Eq. (65), requires only one integration by $\eta$ at each level $m$ in Eq. (71), starting from $m=0$. The zero-order term $\Phi^{(0)}(\eta)$ does not depend on functions $\Psi_{(k)}$ and is determined exclusively by $H(\eta)$, Eq. (55). The zero-order term can be presented as 


$$
\Phi_{n}^{(0)}(\eta)=-\frac{1}{10} g(\eta) \int_{0}^{\eta} d \eta^{\prime} \frac{d h_{n}\left(\eta^{\prime}\right)}{d \eta} e^{-(3 / 10) \tau\left(\eta, \eta^{\prime}\right)}
$$

It is crucial to remember that the function $\Phi(\eta)$, Eq. (54), always contains the narrow visibility function $g(\eta)$ (see Appendix B). In particular, function $\Phi^{(0)}(\eta)$ is nonzero only for $\eta$ within the width of $g(\eta)$, and is proportional to this width. In the era of decoupling, we denote the characteristic width of $g(\eta)$ by $\Delta \eta_{\text {dec }}$. With $\Delta \eta_{\text {dec }}$ we associate the characteristic wave number $n_{*}$ :

$$
n_{*}=\frac{2 \pi}{\Delta \eta_{\mathrm{dec}}}
$$

Numerically, $\Delta \eta_{\mathrm{dec}} \approx 3 \times 10^{-3}$ and $n_{*} \approx 2 \times 10^{3}$. In what follows, we will be interested in CMB multipoles $\ell \lesssim$ $10^{3}$. They are mostly generated by perturbations with wave numbers $n \lesssim 10^{3}$. Therefore, for wave numbers of interest, we regard $n / n_{*}$ as a small parameter.

We shall now show that $\Phi^{(0)}(\eta)$ is the dominant term of the series (65). The next term, $\Phi^{(1)}(\eta) n^{2}$, is at least a factor $\left(n / n_{*}\right)^{2}$ smaller than $\Phi^{(0)}(\eta)$, and so on. The explicit expression for $\Phi^{(1)}$ is as follows

$$
\begin{aligned}
\Phi^{(1)}(\eta)= & -g(\eta) \int_{0}^{\eta} d \eta^{\prime}\left[\omega_{-}(1) \frac{d h\left(\eta^{\prime}\right)}{d \eta^{\prime}}\right. \\
& \left.-\omega_{+}(1) e^{\tau\left(\eta^{\prime}\right)} \Phi^{(0)}\left(\eta^{\prime}\right)\right] e^{-(3 / 10) \tau\left(\eta, \eta^{\prime}\right)} \Psi_{(1)}\left(\eta, \eta^{\prime}\right) .
\end{aligned}
$$
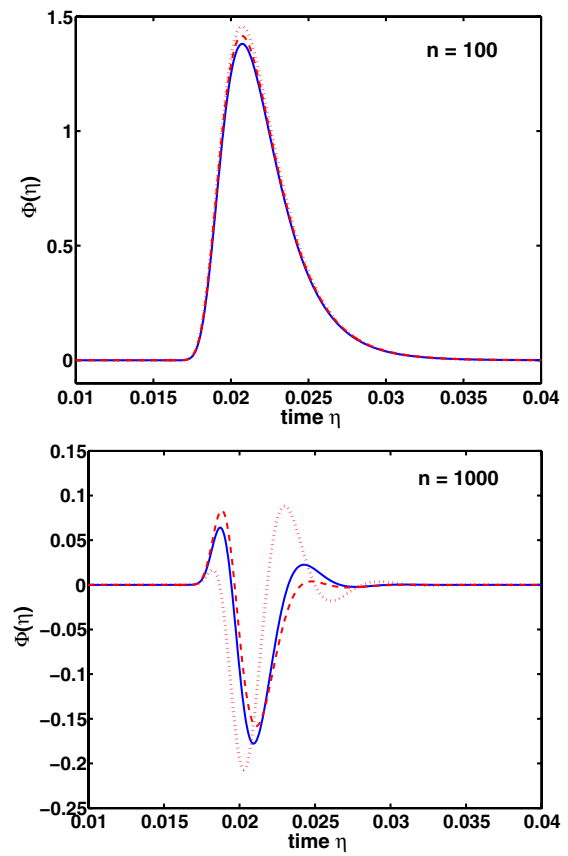

Effectively, function $\Phi^{(1)}(\eta)$, in comparison with $\Phi^{(0)}(\eta)$, contains an extra factor $\Psi_{(1)}\left(\eta, \eta^{\prime}\right)$. Taking into account the fact that the functions $g(\eta)$ and $e^{-(7 / 10) \tau\left(\eta^{\prime \prime}, \eta^{\prime}\right)}$ are localized in the interval of arguments not larger than $\Delta \eta_{\text {dec }}$, this factor evaluates to a number not larger than $\left(\Delta \eta_{\text {dec }}\right)^{2}$. Therefore, the $m=1$ term in Eq. (65) is at least a factor $\left(n / n_{*}\right)^{2}$ smaller than the $m=0$ term.

These analytical evaluations are confirmed by numerical analysis as shown in Fig. 3. The solid line shows the exact numerical solution found from Eq. (61) for $h_{n}(\eta)$ and $q(\eta)$ described in Sec. II B and Appendix B respectively. The dashed line is plotted according to formula (73), with the same $h_{n}(\eta)$ and $q(\eta)$. It is seen from Fig. 3 that the zeroorder term $\Phi^{(0)}(\eta)$ is a good approximation. The deviations are significant, they reach $(20-25) \%$, only for the largest wave numbers $n$ in the domain of our interest.

\section{MULTIPOLE EXPANSION AND POWER SPECTRA OF THE RADIATION FIELD}

\section{A. Multipole coefficients}

Having found $\Phi_{n, s}(\eta)$ for a single gravitational wave specified by Eq. (49) one can find $\alpha$ and $\beta$ functions according to Eqs. (63a) and (63b). Then, using Eqs. (34)-(36), (38), and (50), one can find the multipole coefficients $a_{\ell m}^{X}(X=I, E, B)$ participating in the decompositions (37). Although this route has been traversed before [31], we have made independent calculations in a
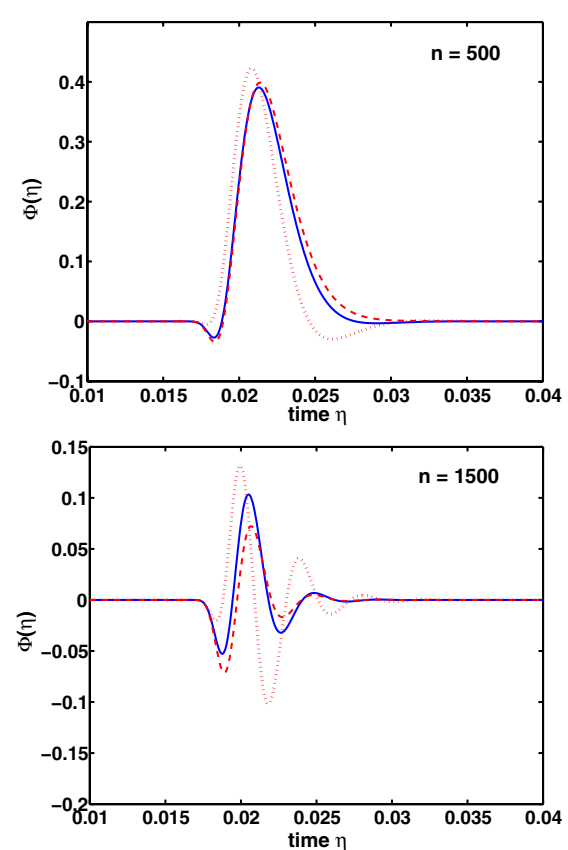

FIG. 3 (color online). Function $\Phi_{n}(\eta)$ for different values of $n$. The solid line is exact numerical solution to Eq. (61). The dashed line is the zero-order approximation (73). The dotted line shows the approximation (91) (see below). The g.w. mode functions are normalized such that $h_{n}\left(\eta_{r}\right)=1$. 
more general arrangement. Formulas derived in this subsection are effectively a confirmation of the correctness of calculations in Ref. [31].

First, we integrate over photon frequencies (for the definition of $\gamma$ see Eq. (B3)) and arrive at the following expressions

$$
\begin{aligned}
I_{n, s}(\mu, \phi) & =\gamma\left[\left(1-\mu^{2}\right) \alpha_{n, s}(\mu) e^{ \pm 2 i \phi}\right], \\
E_{n, s}(\mu, \phi) & =-\gamma\left[\left(1-\mu^{2}\right)\left(\left(1+\mu^{2}\right) \frac{d^{2}}{d \mu^{2}}+8 \mu \frac{d}{d \mu}+12\right) \beta_{n, s}(\mu) e^{ \pm 2 i \phi}\right], \\
B_{n, s}(\mu, \phi) & =\mp \gamma\left[2\left(1-\mu^{2}\right)\left(i \mu \frac{d^{2}}{d \mu^{2}}+4 i \frac{d}{d \mu}\right) \beta_{n, s}(\mu) e^{ \pm 2 i \phi}\right],
\end{aligned}
$$

where, as before, the upper and lower signs correspond to $s=1=L$ and $s=2=R$, respectively. The $\pm 2 \phi$ dependence in Eqs. (74) implies that only the $m= \pm 2$ multipoles are nonzero.

Then, we integrate Eqs. (74) over angular variables in order to find $a_{\ell m}^{X}$ according to Eq. (37). Using the notations $T_{\ell}(\zeta), E_{\ell}(\zeta), B_{\ell}(\zeta)$ for the functions arising in course of calculations (and called multipole projection functions)

$$
\begin{aligned}
T_{\ell}(\zeta) & =\sqrt{\frac{(\ell+2) !}{(\ell-2) !}} \frac{j_{\ell}(\zeta)}{\zeta^{2}}, \\
E_{\ell}(\zeta) & =\left[\left(2-\frac{l(l-1)}{\zeta^{2}}\right) j_{\ell}(\zeta)-\frac{2}{\zeta} j_{\ell-1}(\zeta)\right], \\
B_{\ell}(\zeta) & =2\left[-\frac{(\ell-1)}{\zeta} j_{\ell}(\zeta)+j_{\ell-1}(\zeta)\right],
\end{aligned}
$$

and replacing $\alpha(\mu)$ and $\beta(\mu)$ by their expressions (63), we finally arrive at

$a_{\ell m}^{T}(n, s)=(-i)^{\ell-2}\left(\delta_{2, m} \delta_{1, s}+\delta_{-2, m} \delta_{2, s}\right) a_{\ell}^{T}(n, s)$,

$a_{\ell m}^{E}(n, s)=(-i)^{\ell-2}\left(\delta_{2, m} \delta_{1, s}+\delta_{-2, m} \delta_{2, s}\right) a_{\ell}^{E}(n, s)$,

$a_{\ell m}^{B}(n, s)=(-i)^{\ell-2}\left(\delta_{2, m} \delta_{1, s}-\delta_{-2, m} \delta_{2, s}\right) a_{\ell}^{B}(n, s)$,

where

$a_{\ell}^{T}(n, s)=\gamma \sqrt{4 \pi(2 \ell+1)} \int_{0}^{\eta_{R}} d \eta\left(H_{n, s}(\eta)-\Phi_{n, s}(\eta)\right) T_{\ell}(\zeta)$,

$a_{\ell}^{E}(n, s)=\gamma \sqrt{4 \pi \overline{(2 \ell}+1)} \int_{0}^{\eta_{R}} d \eta \Phi_{n, s}(\eta) E_{\ell}(\zeta)$,

$a_{\ell}^{B}(n, s)=\gamma \sqrt{4 \pi(2 \ell+1)} \int_{0}^{\eta_{R}} d \eta \Phi_{n, s}(\eta) B_{\ell}(\zeta)$.

\section{B. Superposition of gravitational waves with arbitrary wave vectors}

It is important to remember that the result (76) is valid only for a special wave, with the wave vector $\mathbf{n}$ oriented exactly along the coordinate axis $z$. Since the perturbed gravitational field is a random collection of waves with all possible wave vectors $\mathbf{n}$, and we are interested in their summarized effect as seen in some fixed observational direction $\theta, \phi$, we have to find the generalization of Eq. (76) to an arbitrary wave, and then to sum them up.

To find the effect of an arbitrary wave, there is no need to do new calculations. It is convenient to treat calculations in Sec. VA as done in a (primed) coordinate system specially adjusted to a given wave in such a manner that the wave propagates along $z^{\prime}, \mathbf{n}^{\prime}=(0,0, n)$. The observational direction $e^{i}$ is characterized by $\theta^{\prime}, \phi^{\prime}$. The quantities $X=I$, $E, B$ calculated in Sec. VA are functions of $\theta^{\prime}, \phi^{\prime}$ expanded over $Y_{\ell m}\left(\theta^{\prime}, \phi^{\prime}\right)$,

$$
X_{\mathbf{n}^{\prime}, s}\left(\theta, \phi^{\prime}\right)=\sum_{\ell=0}^{\infty} \sum_{m=-\ell}^{\ell} \mathbf{a}_{\ell m}^{X}(n, s) Y_{\ell m}\left(\theta^{\prime}, \phi^{\prime}\right),
$$

where $\mathbf{a}_{\ell m}^{X}$ is a set of coefficients (compare with Eq. (37)): $a_{\ell m}^{T},[(\ell+2) ! /(\ell-2) !]^{1 / 2} a_{\ell m}^{E},[(\ell+2) ! /(\ell-2) !]^{1 / 2} a_{\ell m}^{B}$.

Now, imagine that this special (primed) coordinate system is rotated with respect to the observer's (unprimed) coordinate system by some Euler angles

$$
\alpha=\phi_{\mathbf{n}}, \quad \beta=\theta_{\mathbf{n}}, \quad \gamma=0
$$

(see, for example [41]). The same observational direction $e^{i}$ is now characterized by $\theta, \phi$, and the same wave vector $\mathbf{n}^{\prime}$ is now characterized by the unit vector

$$
\tilde{\mathbf{n}}=\mathbf{n} / n=\left(\sin \theta_{\mathbf{n}} \cos \phi_{\mathbf{n}}, \sin \theta_{\mathbf{n}} \sin \phi_{\mathbf{n}}, \cos \theta_{\mathbf{n}}\right) .
$$

Obviously, the already calculated numerical values of the invariants $X\left(\theta^{\prime}, \phi^{\prime}\right)$ do not depend on the rotation of the coordinate system. Being expressed in terms of $\theta, \phi$, the invariants describe the effect produced by a wave with a given (arbitrary) unit wave vector $\tilde{\mathbf{n}}$, as seen in the direction $\theta, \phi$.

The transformation between coordinate systems $\left(\theta^{\prime}, \phi^{\prime}\right)$ and $(\theta, \phi)$ is accompanied by the transformation of spherical harmonics,

$$
Y_{\ell m}\left(\theta^{\prime}, \phi^{\prime}\right)=\sum_{m^{\prime}=-\ell}^{\ell} D_{m^{\prime}, m}^{\ell}(\tilde{\mathbf{n}}) Y_{\ell m^{\prime}}(\theta, \phi)
$$

where

$$
D_{m^{\prime}, m}^{\ell}(\tilde{\mathbf{n}}) \equiv D_{m^{\prime}, m}^{\ell}\left(\phi_{\mathbf{n}}, \theta_{\mathbf{n}}, 0\right)
$$

are the Wigner symbols [42]. Later, we will need their orthogonality relationship 


$$
\int d \Omega D_{m, p}^{\ell}(\tilde{\mathbf{n}}) D_{m^{\prime}, p}^{\ell^{\prime *}}(\tilde{\mathbf{n}})=\frac{4 \pi}{2 \ell+1} \delta_{\ell \ell^{\prime}} \delta_{m m^{\prime}},
$$

where $d \Omega=\sin \theta_{\mathbf{n}} d \theta_{\mathbf{n}} d \phi_{\mathbf{n}}$.

We can now rewrite Eq. (78) in terms of $\theta, \phi$, and thus find the contribution of a single arbitrary Fourier component,

$X_{\mathbf{n}, s}(\theta, \phi)=\sum_{\ell=0}^{\infty} \sum_{m=-\ell}^{\ell}\left(\sum_{m^{\prime}=-\ell}^{\ell} \mathbf{a}_{\ell m^{\prime}}^{X}(n, s) D_{m m^{\prime}}^{\ell}(\tilde{\mathbf{n}})\right) Y_{\ell m}(\theta, \phi)$.

The superposition of all Fourier components of the perturbed gravitational field gives, at the observer's position $\mathbf{x}=0$ and at $\eta=\eta_{R}$ (see (47)), the final result:

$$
\begin{aligned}
X(\theta, \phi)= & \frac{\mathcal{C}}{(2 \pi)^{3 / 2}} \int_{-\infty}^{+\infty} \frac{d^{3} \mathbf{n}}{\sqrt{2 n}} \\
& \times \sum_{s=1,2}\left[X_{\mathbf{n}, s}(\theta, \phi) c^{s} c_{\mathbf{n}}+X_{\mathbf{n}, s}^{*}(\theta, \phi)^{s^{*}} c_{\mathbf{n}}\right] .
\end{aligned}
$$

From this expression, combined with Eq. (80), one can read off the random multipole coefficients $a_{\ell m}^{X}$ that participate in the expansions (37):

$$
\begin{aligned}
a_{\ell m}^{X}= & \frac{\mathcal{C}}{(2 \pi)^{3 / 2}} \int_{-\infty}^{+\infty} \frac{d^{3} \mathbf{n}}{\sqrt{2 n}} \sum_{s=1,2} \sum_{m^{\prime}=-\ell}^{\ell}\left[a_{\ell m^{\prime}}^{X}(n, s) D_{m, m^{\prime}}^{\ell}(\tilde{\mathbf{n}})^{s} c_{\mathbf{n}}\right. \\
& \left.+(-1)^{m} a_{\ell-m^{\prime}}^{X *}(n, s) D_{m,-m^{\prime}}^{\ell *}(\tilde{\mathbf{n}})^{s^{*}} c_{\mathbf{n}}\right] .
\end{aligned}
$$

Since $X$ is a real field, the multipole coefficients $a_{\ell m}^{X}$ obey the reality conditions

$$
a_{\ell m}^{X *}=(-1)^{m} a_{\ell,-m}^{X}
$$

as is seen directly from (82). The gravitational-wave nature of metric perturbations is encoded in concrete values of the coefficients (37) and (77). But in all other aspects the argumentation presented here is general.

\section{Angular power spectra for temperature and polarization anisotropies}

It follows from Eq. (82) that the statistical properties of the multipole coefficients $a_{\ell m}^{X}$ are fully determined by the statistical properties of the gravitational-field perturbations represented by the random coefficients $\stackrel{s}{c_{\mathbf{n}}}$. A particular realization of $\stackrel{s}{c_{\mathbf{n}}}$ is responsible for the particular realization of $a_{\ell m}^{X}$ actually observed in the sky. Having derived the distribution function for ${ }_{c}^{s}$ from some fundamental considerations (for example, from the assumption of the initial quantum-mechanical vacuum state of perturbations) we could estimate the probability of the observed set $a_{\ell m}^{X}$ within the ensemble of all possible sets. We could also evaluate the inevitable uncertainty in the observational determination of the parameters of the underlying random process. This uncertainty is associated with the inherent absence of ergodicity of any random process on a 2-sphere (i.e. sky) [43]. In this paper, however, we adopt a minimalistic approach; we postulate only the relationships (16) and calculate only the quadratic correlation functions for $a_{\ell m}^{X}$.

Clearly, the mean values of the multipole coefficients are zeros,

$$
\left\langle a_{\ell m}^{X}\right\rangle=\left\langle a_{\ell m}^{X *}\right\rangle=0
$$

To calculate the variances and cross-correlation functions, we have to form the products $a_{\ell m}^{X *} a_{\ell^{\prime} m^{\prime}}^{X^{\prime}}$ and then take their statistical averages. First, we find

$$
\begin{aligned}
\left\langle a_{\ell m}^{X *} a_{\ell^{\prime} m^{\prime}}^{X^{\prime}}\right\rangle= & \frac{\mathcal{C}^{2}}{(2 \pi)^{3}} \int \frac{n^{2} d n d \Omega}{2 n} \sum_{s=1,2} \sum_{m_{1}=-\ell}^{\ell} \sum_{m_{1}^{\prime}=-\ell}^{\ell}\left[a_{\ell m_{1}}^{X *}(n, s) a_{\ell^{\prime} m_{1}^{\prime}}^{X^{\prime}}(n, s) D_{m m_{1}}^{\ell *}(\tilde{\mathbf{n}}) D_{m^{\prime} m_{1}^{\prime}}^{\ell^{\prime}}(\tilde{\mathbf{n}})\right. \\
& \left.+a_{\ell m_{1}}^{X}(n, s) a_{\ell^{\prime} m_{1}^{\prime}}^{X^{\prime *}}(n, s) D_{-m, m_{1}}^{\ell}(\tilde{\mathbf{n}}) D_{-m^{\prime}, m_{1}^{\prime}}^{\ell^{\prime} *}(\tilde{\mathbf{n}})\right] .
\end{aligned}
$$

We now take into account the fact (compare with Eq. (76)) that

$$
a_{\ell m_{1}}^{X}(n, s) a_{\ell^{\prime} m_{1}^{\prime}}^{X^{\prime}}(n, s) \propto \delta_{m_{1} m_{1}^{\prime}}
$$

This property allows us to get rid of summation over $m_{1}^{\prime}$ in Eq. (84). Then, we perform integration over $d \Omega$ and use the orthogonality relationships (79). We finally arrive at

$$
\left\langle a_{\ell m}^{X *} a_{\ell^{\prime} m^{\prime}}^{X^{\prime}}\right\rangle=C_{\ell}^{X X^{\prime}} \delta_{\ell \ell^{\prime}} \delta_{m m^{\prime}},
$$

where

$$
\begin{aligned}
C_{\ell}^{X X^{\prime}}= & \frac{\mathcal{C}^{2}}{4 \pi^{2}(2 \ell+1)} \int n d n \sum_{s=1,2} \sum_{m=-\ell}^{\ell}\left(a_{\ell m}^{X}(n, s) a_{\ell m}^{X^{\prime} *}(n, s)\right. \\
& \left.+a_{\ell m}^{X *}(n, s) a_{\ell m}^{X^{\prime}}(n, s)\right) .
\end{aligned}
$$

Other quadratic averages, such as $\left\langle a_{\ell m}^{X} a_{\ell^{\prime} m^{\prime}}^{X^{\prime}}\right\rangle,\left\langle a_{\ell m}^{X *} a_{\ell^{\prime} m^{\prime}}^{X^{\prime} *}\right\rangle$, follow from (85) and the reality condition (83).

The angular correlation and cross-correlation functions of the fields $I, E, B$ are directly expressible in terms of Eq. (86). For example,

$$
\left\langle I\left(\theta_{1}, \phi_{1}\right) I\left(\theta_{2}, \phi_{2}\right)\right\rangle=\Gamma(\delta)=\sum_{\ell=0}^{\infty} \frac{2 \ell+1}{4 \pi} C_{\ell}^{T T} P_{\ell}(\cos \delta),
$$


where $\delta$ is the angular separation between the directions $\left(\theta_{1}, \phi_{1}\right)$ and $\left(\theta_{2}, \phi_{2}\right)$ on the sky. If the actually measured values of $a_{\ell m}^{X}$ represent a particular realization of a Gaussian random process, quantities $C_{\ell}^{X X^{\prime}}$, constructed from the measured $a_{\ell m}^{X}$ according to the r.h.s. of Eq. (86), are the best unbiased estimates [43].

One can note that the final result (85) and (86) is the integral of individual contributions (76) from single gravitational waves given in a special frame discussed in Sec. VA. However, one cannot jump directly from (76) to (86) (which is often done in the literature). In general, Eq. (86) does not follow from Eq. (76). By calculations given in this subsection we have rigorously shown that Eq. (86) is justified only if special statistical assumptions (16) are adopted.

One can also note that the correlation functions containing the label $B$ once, i.e. $C_{\ell}^{T B}$ and $C_{\ell}^{E B}$, vanish if the extra assumptions (18) and (23) are made. Indeed, under these assumptions one can use one and the same mode function for both polarization states $s$, Eq. (24). Then, the coefficients $a_{\ell m}^{B}(n, s)$, Eq. (76c), differ essentially only in sign for two different $s$, i.e. $a_{\ell 2}^{B}(n, L)=-a_{\ell,-2}^{B}(n, R)$. Therefore, their contributions will cancel out in expressions (86) for $C_{\ell}^{T B}$ and $C_{\ell}^{E B}$. This statement is in agreement with Ref. [44].

Without having access to a theory of everything which could predict one unique distribution of the CMB radiation field over the sky, we have to rely on the calculated statistical averages (86). We can also hope that our universe is a "typical" one, so that the observed values of the correlation functions should not deviate too much from the statistical mean values.

\section{EFFECTS OF RECOMBINATION ERA}

All our final graphs and physical conclusions in this paper are based on exact formulas and numerical calculations, starting from numerical representation of the key functions $H(\eta)$ and $\Phi(\eta)$, Eqs. (55) and (61). However, we derive and explain all our results by developing manageable and accurate analytical approximations. At every level of calculations we compare exact numerical results with analytical ones.

\section{A. Temperature anisotropy angular power spectrum}

The temperature anisotropy power spectrum $C_{\ell}^{T T}$ is determined by the multipole coefficients $a_{\ell}^{T}(n, s)$, Eqs. (86), (76a), and (77a). The typical graphs for the functions $H_{n, s}(\eta)$ and $\Phi_{n, s}(\eta)$ are shown in Fig. 4.

Since the visibility function $g(\eta)$ is a narrow function, a convenient analytical approximation is the limit of an instantaneous recombination. The function $e^{-\tau}$ is replaced by a step function changing from 0 to 1 at $\eta=\eta_{\mathrm{dec}}, e^{-\tau}=$ $h\left(\eta-\eta_{\mathrm{dec}}\right)$, and the function $g(\eta)$ is replaced by a deltafunction, $g(\eta)=\delta\left(\eta-\eta_{\mathrm{dec}}\right)$. In this limit, the contribution to $a_{\ell}^{T}(n, s)$ from the scattering term $\Phi_{n, s}^{(0)}(\eta)$ is proportional to $\Delta \eta_{\mathrm{dec}}$. It can be neglected in comparison with the contribution from the gravitational term $H_{n, s}(\eta)$. The ratio of these contributions is of the order of $n \Delta \eta_{\mathrm{rec}}$, and it tends to zero in the limit of instantaneous recombination, $\Delta \eta_{\mathrm{dec}} \rightarrow 0$.

Neglecting the scattering term, we write

$$
a_{\ell}^{T}(n, s)=\gamma \sqrt{4 \pi(2 \ell+1)} \int_{\eta_{\mathrm{dec}}}^{\eta_{R}} d \eta \frac{d h_{n}}{d \eta} T_{\ell}(\zeta)
$$

This integral can be taken by parts,

$$
\begin{aligned}
a_{\ell}^{T}(n, s)= & \gamma \sqrt{4 \pi(2 \ell+1)}\left[-\stackrel{s}{h}_{n}\left(\eta_{\mathrm{dec}}\right) T_{\ell}\left(\zeta_{\mathrm{dec}}\right)\right. \\
& \left.+\int_{\eta_{\mathrm{dec}}}^{\eta_{R}} d \eta n h_{n}(\eta) \frac{d T_{\ell}(\zeta)}{d \zeta}\right] .
\end{aligned}
$$

The remaining integral contains oscillating functions and its value is smaller, for sufficiently large $n$ 's, than the value of the integrated term. This is illustrated in Fig. 5. Therefore, we have

$$
a_{\ell}^{T}(n, s)=-\gamma \sqrt{4 \pi(2 \ell+1)} h_{n}\left(\eta_{\mathrm{dec}}\right) T_{\ell}\left(\zeta_{\mathrm{dec}}\right) .
$$

Finally, we put Eq. (88) into Eq. (86) and take into account the definition of the metric power spectrum (13). Then, we get

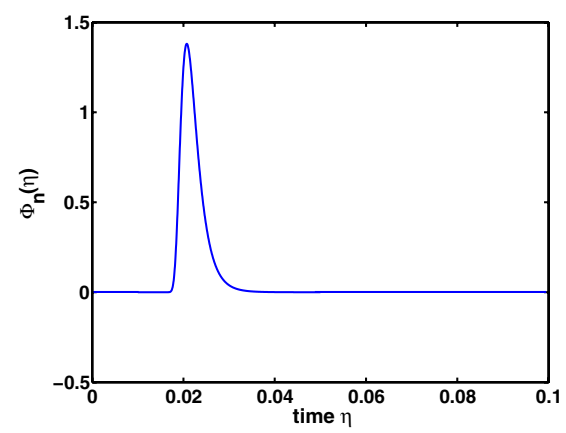

FIG. 4 (color online). The source functions $H_{n, s}(\eta)$ and $\Phi_{n, s}(\eta)(n=100)$ of temperature and polarization anisotropies (the normalization is chosen such that $\left.h_{n}\left(\eta_{r}\right)=1\right)$. 

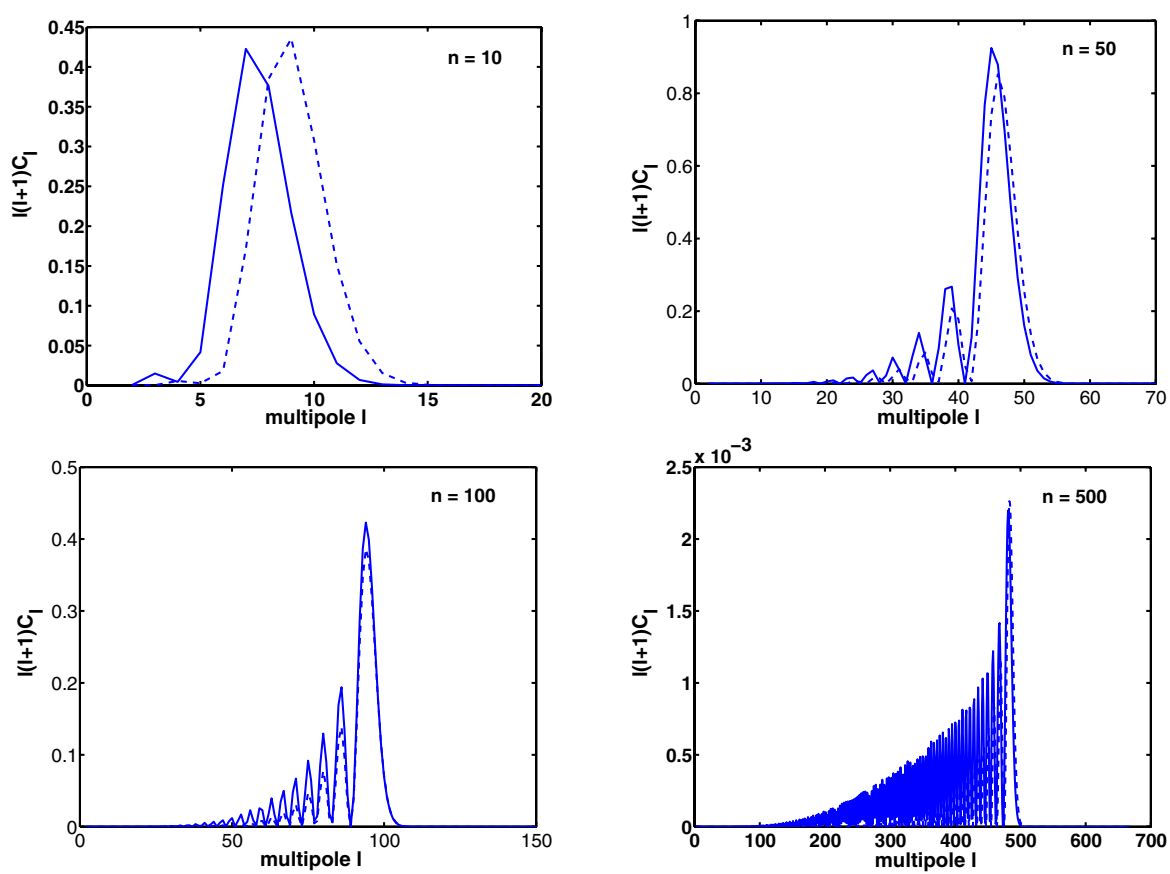

FIG. 5 (color online). The contributions to the power spectrum $\ell(\ell+1) C_{\ell}^{T T}$ from an individual mode $n$. The solid line shows the exact result calculated according to (87), while the dashed line shows the approximation (88). The normalization has been chosen such that $h_{n}\left(\eta_{r}\right)=1$.

$$
C_{\ell}^{T T}=4 \pi \gamma^{2} \int \frac{d n}{n} h^{2}\left(n, \eta_{\mathrm{dec}}\right) T_{\ell}^{2}\left(\zeta_{\mathrm{dec}}\right)
$$

The projection factor $T_{\ell}^{2}\left(\zeta_{\text {dec }}\right)$ is given by Eq. (75a). Since the spherical Bessel functions reach maximum when the argument and the index are approximately equal, $\zeta_{\text {dec }} \approx \ell$, a particular wave number $n$ is predominantly projected onto the multipole $\ell \approx n$ :

$$
\ell \approx \zeta_{\mathrm{dec}}=n\left(\eta_{R}-\eta_{\mathrm{dec}}\right) \approx n .
$$

This can also be seen in Fig. 5. Thus, the oscillatory features of the metric power spectrum $h^{2}\left(n, \eta_{\text {dec }}\right)$ in the $n$-space are fully responsible for the oscillatory features of the angular power spectrum $\ell(\ell+1) C_{\ell}^{T T}$ in the $\ell$-space [11]. (We use this opportunity to correct a misprint in Fig. 2 of Ref. [11]: the plotted lines are functions $C_{l}$, not $l(l+$ 1) $C_{l}$. For the early graphs of $C_{\ell}$ see Ref. [45].)

The g.w. metric power spectrum $h^{2}\left(n, \eta_{\mathrm{dec}}\right)$ for the case $\beta=-2$, and the function $\ell(\ell+1) C_{\ell}^{T T}$ caused by this spectrum, are shown in Fig. 7(b) and 7(a). The normalization of the metric power spectrum is such that the function $\ell(\ell+1) C_{\ell}^{T T}$ at $\ell=2$ is equal to $1326 \mu \mathrm{K}^{2}$ [19]. The interval $\ell \leqslant 90$ is generated by waves with $n \lesssim 90$. These waves did not enter the Hubble radius by the time $\eta_{\text {dec }}$. Their amplitudes are approximately equal for all $n$ 's in this interval (compare with Fig. 1). The gradual decrease of the angular power spectrum at larger $\ell$ 's is the reflection of the gradual decrease of power in shorter gravitational waves whose amplitudes have been adiabatically decreas- ing since the earlier times when the waves entered the Hubble radius.

\section{B. Polarization anisotropy angular power spectrum}

The decisive function for polarization calculations is $\Phi_{n, s}(\eta)$. We have approximated this function by $\Phi_{n, s}^{(0)}$, Eq. (73), and compared it with exact result in Fig. 3. For qualitative derivations it is useful to make further simplifications.

Since $g(\eta)$ is a narrow function, the integral in Eq. (73) is effective only within a narrow interval $\Delta \eta_{\mathrm{dec}}$. Assuming that the function $d h_{n}(\eta) / d \eta$ does not vary significantly within this interval, we can take this function from under the integral,

$$
\Phi_{n, s}^{(0)}(\eta) \approx-\frac{1}{10} \frac{d h_{n}(\eta)}{d \eta}\left(g(\eta) \int_{0}^{\eta} d \eta^{\prime} e^{-(3 / 10) \tau\left(\eta, \eta^{\prime}\right)}\right) .
$$

Clearly, the assumption that the function $d h_{n}(\eta) / d \eta$ is almost constant within the window $\Delta \eta_{\text {dec }}$ gets violated for sufficiently short waves. With (91), we expect degradation of accuracy for wave numbers $n$ approaching $n_{*}$. This is illustrated by a dotted line in Fig. 3. Nevertheless, the approximation (91) is robust for $n \lesssim n_{*}$, and it reveals the importance of first derivatives of metric perturbations for evaluation of the CMB polarization.

We now introduce the symbol $P$ to denote either $E$ or $B$ components of polarization. In terms of multipoles 
$a_{\ell}^{P}(n, s)$, the angular power spectrum is given by Eq. (86). Putting (91) into Eqs. (77b) and (77c) and denoting by $P_{\ell}(\zeta)$ the respective projection functions, we get

$$
\begin{aligned}
a_{\ell}^{P}(n, s) \approx & \gamma \sqrt{4 \pi(2 \ell+1)} \int_{0}^{\eta_{R}} d \eta\left(-\frac{1}{10} \frac{d s h_{n}(\eta)}{d \eta} P_{\ell}(\zeta)\right) \\
& \times\left[g(\eta) \int_{0}^{\eta} d \eta^{\prime} e^{-(3 / 10) \tau\left(\eta, \eta^{\prime}\right)}\right] .
\end{aligned}
$$

Again referring to the peaked character of $g(\eta)$ and assuming that the combination $\left(d h_{n}^{s}(\eta) / d \eta\right) P_{\ell}(\zeta)$ does not change significantly within the window $\Delta \eta_{\mathrm{dec}}$, we take this combination from under the integral,

$$
\begin{aligned}
a_{\ell}^{P}(n, s) \approx & \gamma \sqrt{4 \pi(2 \ell+1)} D(n) \\
& \times\left.\left(-\frac{1}{10} \frac{d h_{n}(\eta)}{d \eta} P_{\ell}(\zeta)\right)\right|_{\eta=\eta_{\mathrm{rec}}} \Delta .
\end{aligned}
$$

The two new factors in this expression, $D(n)$ and $\Delta$, require clarification.

The factor $D(n)$ compensates for gradual worsening of our approximation when the wave number $n$ approaches $n_{*}$. For large $n$ 's, the functions under the integrals change sign within the window $\Delta \eta_{d e c}$, instead of being constant there. This leads to the decrease of the true value of the integral in comparison with the approximated one. The evaluation of this worsening suggests that it can be described by the damping factor

$$
D(n) \equiv\left[1+\left(\frac{n \Delta \eta_{\mathrm{dec}}}{2}\right)^{2}\right]^{-1} .
$$

We inserted this factor by hand in Eq. (93) Additional arguments on this damping are given in Ref. [46] (see also [47]).

The factor $\Delta$ is the result of the remaining integration over $\eta$ in Eq. (92) [33],

$$
\begin{aligned}
\Delta & \equiv \int_{0}^{\eta_{R}} d \eta g(\eta)\left(\int_{0}^{\eta} d \eta^{\prime} e^{-(3 / 10) \tau\left(\eta, \eta^{\prime}\right)}\right) \\
& =\frac{10}{7} \int_{0}^{\eta_{R}} d \eta\left[e^{-(3 / 10) \tau(\eta)}-e^{-\tau(\eta)}\right] .
\end{aligned}
$$

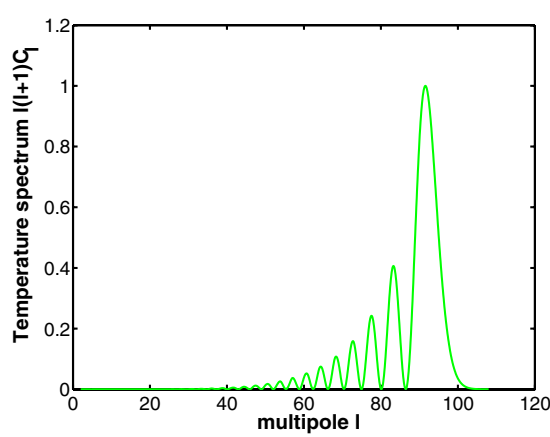

Since $e^{-\tau}$ rapidly changes from 0 to 1 around recombination, the integrand $\left(e^{-(3 / 10) \tau(\eta)}-e^{-\tau(\eta)}\right)$ is nonzero only there, and $\Delta$ is expected to be of the order of $\Delta \eta_{\mathrm{dec}}$. To give a concrete example, we approximate $g(\eta)$ by a Gaussian function

$$
g(\eta)=\frac{1}{\sqrt{2 \pi}\left(\Delta \eta_{\mathrm{dec}} / 2\right)} \exp \left(-\frac{\left(\eta-\eta_{\mathrm{dec}}\right)^{2}}{2\left(\Delta \eta_{\mathrm{dec}} / 2\right)^{2}}\right)
$$

Then, the quantity $\Delta$ can be found exactly,

$$
\begin{aligned}
\Delta= & \frac{5}{7} \Delta \eta_{\mathrm{dec}} \int_{-\infty}^{+\infty} d x\left[\left(\frac{1}{2}+\frac{1}{2} \operatorname{erf}\left(\frac{x}{\sqrt{2}}\right)\right)^{3 / 10}\right. \\
& \left.-\left(\frac{1}{2}+\frac{1}{2} \operatorname{erf}\left(\frac{x}{\sqrt{2}}\right)\right)\right] \approx 0.96 \Delta \eta_{\mathrm{dec}} .
\end{aligned}
$$

Clearly, factor $\Delta$ in Eq. (93) demonstrates the fact that the $\mathrm{CMB}$ polarization is generated only during a short interval of time around recombination.

Finally, substituting (93) into (86) and recalling (14), we obtain the polarization angular power spectrum:

$$
\begin{aligned}
C_{\ell}^{P P} \approx & 2 \pi \gamma^{2} \frac{1}{100} \Delta^{2} \int \frac{d n}{n} D^{2}(n) \\
& \times\left.\sum_{s=1,2}\left|\frac{d h(n, \eta)}{d \eta}\right|_{\eta=\eta_{\mathrm{rec}}}\right|^{2} P_{\ell}^{2}\left(\zeta_{\mathrm{rec}}\right) .
\end{aligned}
$$

Similarly to the case of temperature anisotropies, the projection factors $P_{\ell}\left(\zeta_{\mathrm{dec}}\right)$ predominantly translate $n$ into $\ell$ according to Eq. (90). The oscillatory features of the power spectrum of the first time-derivative of metric perturbations get translated into the oscillatory features of the power spectra for $E$ and $B$ components of polarization. This is illustrated in Fig. 7(c) and 7(d). The waves with $n \eta_{\text {dec }} \ll$ $\pi$ did not enter the Hubble radius by $\eta=\eta_{\text {dec }}$. They have no power in the spectrum of $d h(n, \eta) / d \eta$ at $n \ll 90$, and therefore there is no power in polarization at $\ell \ll 90$. On the other hand, the first gravitational peak at $n \approx 90$ gets reflected in the first polarization peak at $\ell \approx 90$.

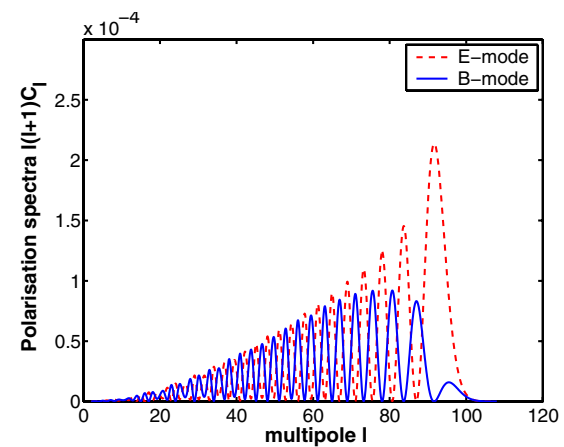

FIG. 6 (color online). Relative contributions of an individual Fourier mode $n=100$ to various multipoles $\ell$ in the power spectra $\ell(\ell+1) C_{\ell}$ for temperature and polarization. The normalization has been chosen arbitrarily but same in both the graphs. 

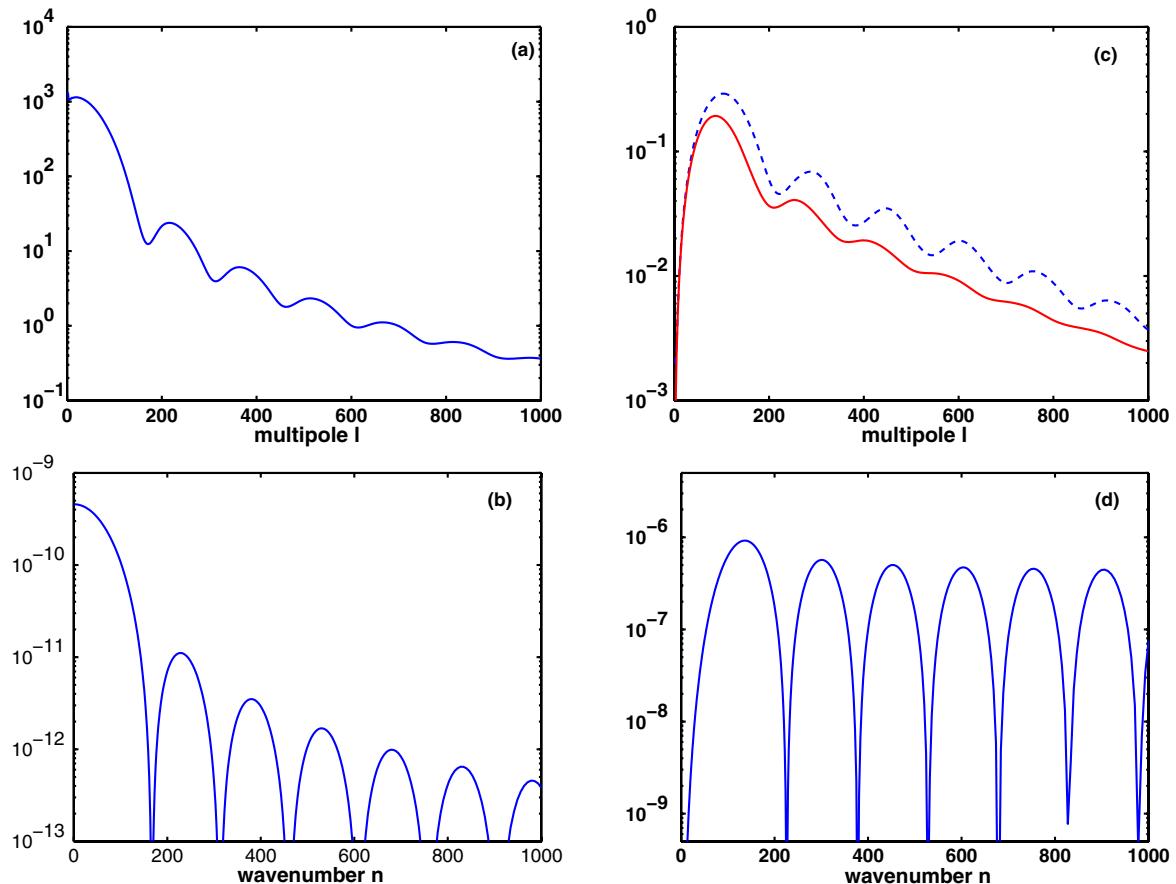

FIG. 7 (color online). The left panel shows (a) the power spectrum of temperature anisotropies $\ell(\ell+1) C_{\ell}^{T T}$ (in $\mu \mathrm{K}^{2}$ ) generated by (b) the power spectrum of g.w. metric perturbations (13), $\beta=-2$. The right panel shows (c) the power spectra of polarization anisotropies $\ell(\ell+1) C_{\ell}^{B B}$ (solid line) and $\ell(\ell+1) C_{\ell}^{E E}$ (dashed line), panel (d) shows the power spectrum of the first time derivative of the same g.w. field, Eq. (15).

There is certain difference, however, between the projection functions $E_{\ell}\left(\zeta_{\mathrm{dec}}\right)$ and $B_{\ell}\left(\zeta_{\mathrm{dec}}\right)$. This is shown in Fig. 6. The $B$-mode projections are more "smeared" and their maxima are shifted to somewhat lower $\ell$ 's. This explains the visible difference between $C_{\ell}^{E E}$ and $C_{\ell}^{B B}$ in Fig. 7 (see also [46]). The polarization angular power spectra, plotted in Fig. 7, were found from exact numerical calculations.

\section{Temperature-polarization cross correlation}

A very special role belongs to the $T E$ cross-correlation spectrum. We will show below that the $T E$ correlation at lower $\ell$ 's must be negative for gravitational waves and positive for density perturbations. This distinctive signature can turn out to be more valuable for identification of relic gravitational waves than the presence of the $B$ polarization in the case of gravitational waves and its absence in the case of density perturbations. The expected $T E$ signal from gravitational waves is about 2 orders of magnitude stronger than the $B B$ signal, and it is much easier to measure. At lower $\ell$ 's, the contributions to $T E$ from gravitational waves and density perturbations are comparable in absolute value, so the g.w. contribution is not a small effect. The total $T E$ cross-correlation has already been measured at some level $[9,48]$.

To find the $T E$ power spectrum we have to use the product of $a_{\ell m}^{T}(n, s)$ and $a_{\ell m}^{E}(n, s)$ in Eq. (86). For qualita- tive analysis we will operate with the approximate expressions (88) and (93). Then, the $T E$ correlation reads

$$
\begin{aligned}
C_{\ell}^{T E} \approx & \pi \gamma^{2}\left(\frac{\Delta}{10}\right) \int \frac{d n}{n} D(n) \sum_{s=1,2}\left(s h^{*}(n, \eta) \frac{d s h(n, \eta)}{d \eta}\right. \\
& \left.+\operatorname{sh}(n, \eta) \frac{d s h^{*}(n, \eta)}{d \eta}\right)\left.\right|_{\eta=\eta_{\mathrm{dec}}}\left(T_{\ell}\left(\zeta_{\mathrm{dec}}\right) E_{\ell}\left(\zeta_{\mathrm{dec}}\right)\right) .
\end{aligned}
$$

For a given $\ell$, the projection factor $\left(T_{\ell}\left(\zeta_{\mathrm{dec}}\right) E_{\ell}\left(\zeta_{\mathrm{dec}}\right)\right)$ peaks at $n \approx \ell$ and is positive there. Therefore, the sign of $C_{\ell}^{T E}$ is determined by the sign of the term:

$$
\begin{aligned}
& \left.\frac{1}{2} \sum_{s=1,2}\left(\stackrel{s *}{h}(n, \eta) \frac{d h(n, \eta)}{d \eta}+\stackrel{s}{h}(n, \eta) \frac{d h^{s *}(n, \eta)}{d \eta}\right)\right|_{\eta=\eta_{\mathrm{dec}}} \\
& \quad=\left.\left(\frac{d h^{2}(n, \eta)}{d \eta}\right)\right|_{\eta=\eta_{\mathrm{dec}}}
\end{aligned}
$$

The adiabatic decrease of the g.w. amplitude upon entering the Hubble radius is preceded by the monotonic decrease of the g.w. mode function $h_{n}(\eta)$ as a function of $\eta$. This behavior is illustrated in Fig. 1. It is clear from the graph that for $n \leqslant 100$ the quantity (96) is negative, because the first derivative of $\stackrel{s}{h}_{n}(\eta)$ is negative. Therefore, for $\ell \lesssim 90$ the correlation $C_{\ell}^{T E}$ must be negative. For larger $\ell$ 's the $T E$ correlation goes through zero, changes sign and 

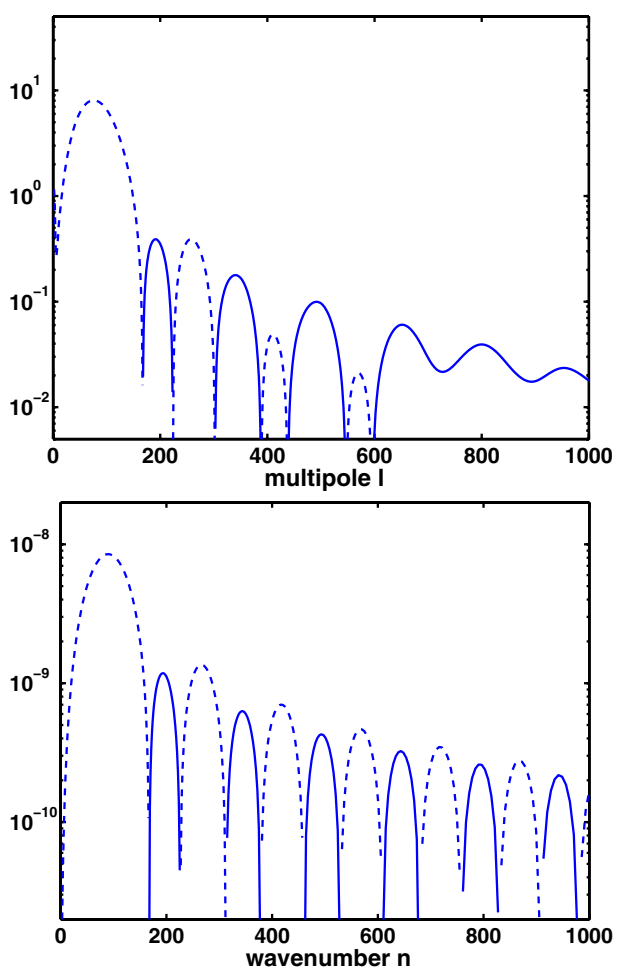

FIG. 8 (color online). The bottom panel shows the spectrum (96) of gravitational waves, whereas the top panel shows the angular power spectrum $\ell(\ell+1) C_{\ell}^{T E}$ caused by these waves. The negative values of these functions are depicted by broken lines.

oscillates reflecting the oscillations of the function (96) in the $n$-space. An exact numerical graph for $T E$ correlation caused by gravitational waves is depicted in Fig. 8. The graph clearly shows how the sign and features of the spectrum (96) get translated into the sign and features of the $T E$ correlation.

It is shown in Appendix D that in the case of density perturbations the $T E$ correlation must be positive at lower $\ell$ 's. This is because the relevant metric perturbations associated with density perturbations are growing in time and therefore the first time-derivative of metric perturbations is positive. Because of other contributions, the $T E$ correlation is expected to change sign at $\ell \approx 70[18,48]$. The region of intermediate multipoles $15 \leq \ell \leq 90$ should be of a particular interest. On one hand, the multipoles $\ell>15$ are not affected by the reionization era and its uncertain details, except the overall suppression by $e^{-2 \tau_{\text {reion }}}$. On the other hand, at $\ell<90$ the g.w. contribution to $T E$ is not much smaller numerically than the contribution from density perturbations. The lower multipoles $\ell \lesssim 15$ are affected by reionization, and we shall study reionization in the next Section.

\section{EFFECTS OF REIONIZATION ERA}

The reionization of the intergalactic medium by first sources of light has occurred relatively late, at $z \sim 30-$ 7 (see, for example, [9,49]). In contrast to the recombination part of the visibility function $g(\eta)$, which is narrow and high, the reionization part of $g(\eta)$ is broad and much
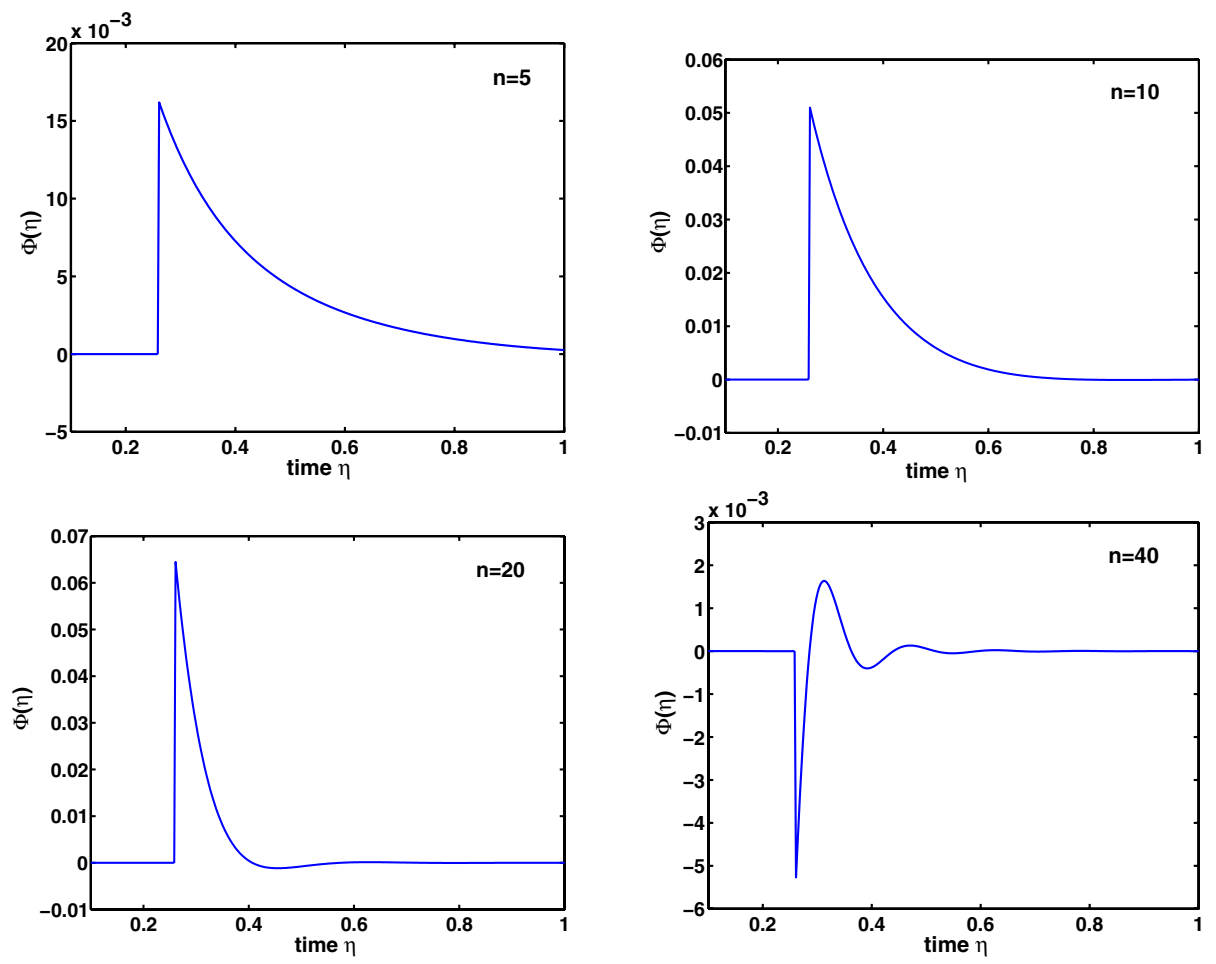

FIG. 9 (color online). The source function $\Phi_{n}(\eta)$ in reionization era for different wave numbers. 

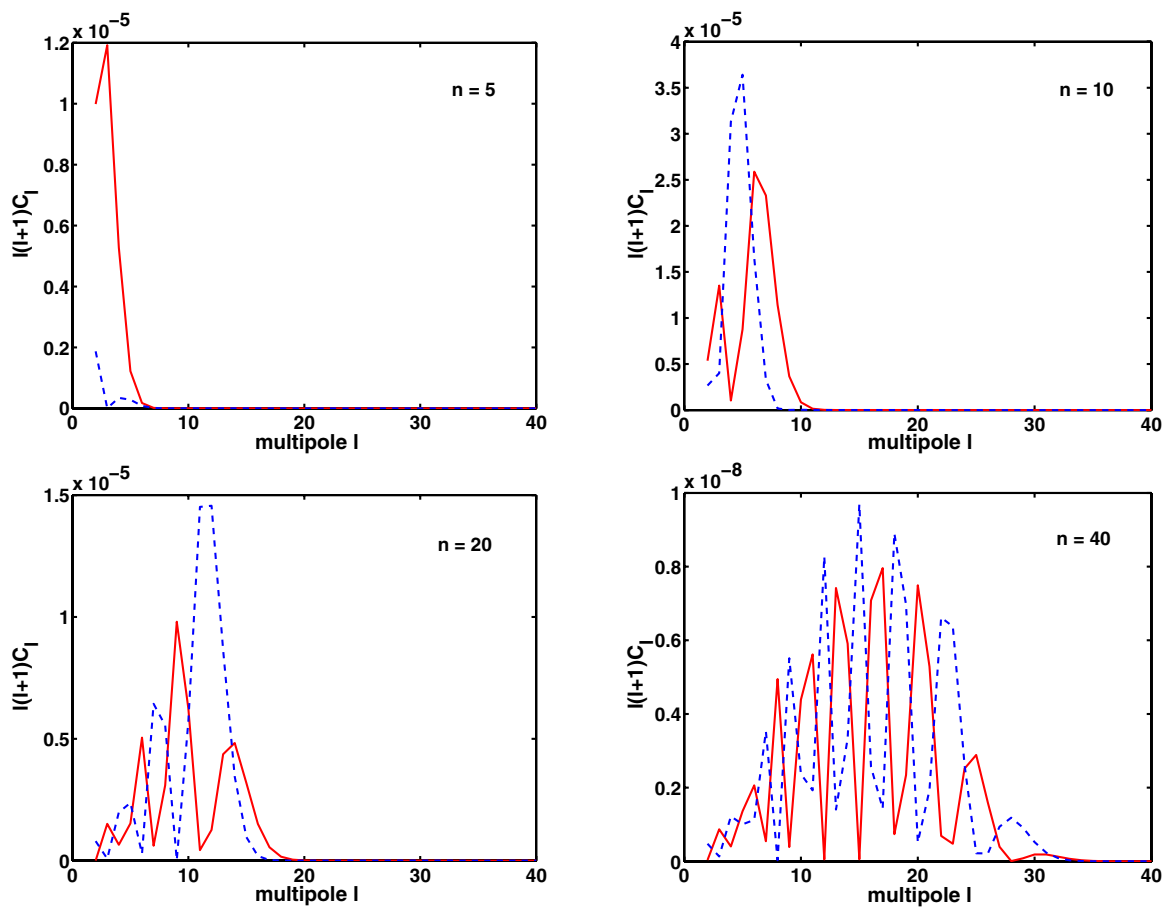

FIG. 10 (color online). The contributions to the angular power spectra from an individual mode $n$. The dashed line is for $\ell(\ell+$ 1) $C_{\ell}^{E E}$ and the solid line is for $\ell(\ell+1) C_{\ell}^{B B}$.

lower (see Fig. 15). For a crude qualitative analysis, one can still apply analytical formulas derived for recombination. One has to replace $\eta_{\text {dec }}$ with $\eta_{\text {reion }}$ and $\Delta \eta_{\text {dec }}$ with $\Delta \eta_{\text {reion. }}$. The waves that start entering the Hubble radius at $\eta_{\text {reion }}$, i.e. waves with wave numbers $n \eta_{\text {reion }} \sim \pi$, provide the most of power to the spectrum of first time derivative of metric perturbations. Therefore, these waves, with $n \sim 12$, produce a "bump" in polarization spectra at the projected $\ell \sim 6$. However, these wave numbers are comparable with the wave number $n_{* *}, n_{* *} \Delta \eta_{\text {reion }} \sim 2 \pi$, that characterizes

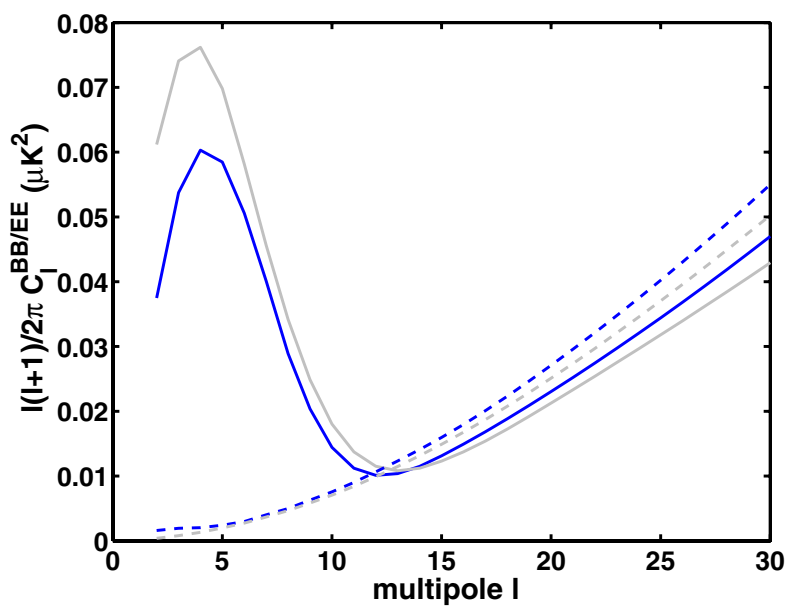

FIG. 11 (color online). The reionization bump in the polarization power spectra. The dashed lines show the spectra when the reionization era is ignored. The solid lines include both eras. The darker lines are for $B$-mode, and the lighter lines for $E$-mode. the width of the visibility function at reionization. The assumption $n \ll n_{* *}$ is not well satisfied, the analytical approximation becomes crude, and one has to rely mostly on numerics for more accurate answers.

The numerical solution to the integral Eq. (61) in the reionization era is shown in Fig. 9. One can see that the damping effect is expected to commence from $n \gtrsim 20$, as the polarization source function $\Phi_{n}(\eta)$ begins to show an oscillatory behavior. The projection relationships are also far away from the almost one-to-one correspondence $\ell \approx$ $n$ that was typical for recombination era. In Fig. 10 we show the contributions of a given $n$ to various $\ell$ 's in the polarization power spectra $\ell(\ell+1) C_{\ell}$. One can see that a considerable portion of power from a given $n$ is distributed over many lower-order $\ell$ 's.

The total effect of the reionization era is shown in Fig. 11. This numerical result was based on our simplified model of homogeneous reionization, as described in Appendix B. The reionization bumps at lower multipoles for $E$ and $B$ components of polarization are similar in shape and numerical value.

\section{COMPARISON WITH AVAILABLE OBSERVATIONS; SIGNATURES OF RELIC GRAVITATIONAL WAVES}

The theory that we are using here is applicable to any primordial spectral index $\mathrm{n}$. The initial conditions for gravitational waves, Eqs. (29) and (30), as well as analogous initial conditions for density perturbations, hold $\beta$, 
and hence $\mathrm{n}$, as a free constant parameter. The spectral index $\mathrm{n}$ can be larger, equal, or smaller than 1 . However, from the theoretical point of view, the "red" primordial spectra $\mathrm{n}<1(\beta<-2)$ seem to be unacceptable, or at least questionable. If $\beta<-2$, the mean-square fluctuations of gravitational field, Eq. (12), are power-law divergent in the limit of very small wave numbers $n$. One could argue that the extrapolation of the primordial spectrum to the "infrared" region of very small $n$ 's is uncertain, and for some reason the shape of the spectrum bends in the infrared region making the integral (12) convergent at the lower limit. We prefer not to hide behind this possibility. If the shape of the primordial spectrum is allowed to be varied, then practically anything in the CMB data can be explained by the properly adjusted primordial spectrum. Therefore, our theoretical preference (unless the data will enforce us to change this preference) is a constant primordial spectral index $\mathrm{n}>1(\beta>-2)$. Obviously, such primordial spectra entail no difficulty in the "ultraviolet" region of very large $n$ 's, because such short-wavelength fluctuations (today's wavelength $\sim 3 \mathrm{~cm}$.) did not satisfy the requirements of superadiabatic amplification and simply have not been generated.

We now return to the $T E$ correlation. In Fig. 12 we show the contributions to the $T E$ correlation function from relic gravitational waves (gw) and primordial density perturbations (dp). (In order to enhance lowest $\ell$ 's we use the combination $(\ell+1) C_{\ell}$ rather than $\ell(\ell+1) C_{\ell}$.) For this illustration, we choose a flat primordial spectrum $\mathrm{n}=1$ $(\beta=-2)$ and assume equal contributions from (gw) and (dp) to the temperature quadrupole: $R=1$, where

$$
R \equiv C_{\ell=2}^{T T}(g w) / C_{\ell=2}^{T T}(d p) \text {. }
$$

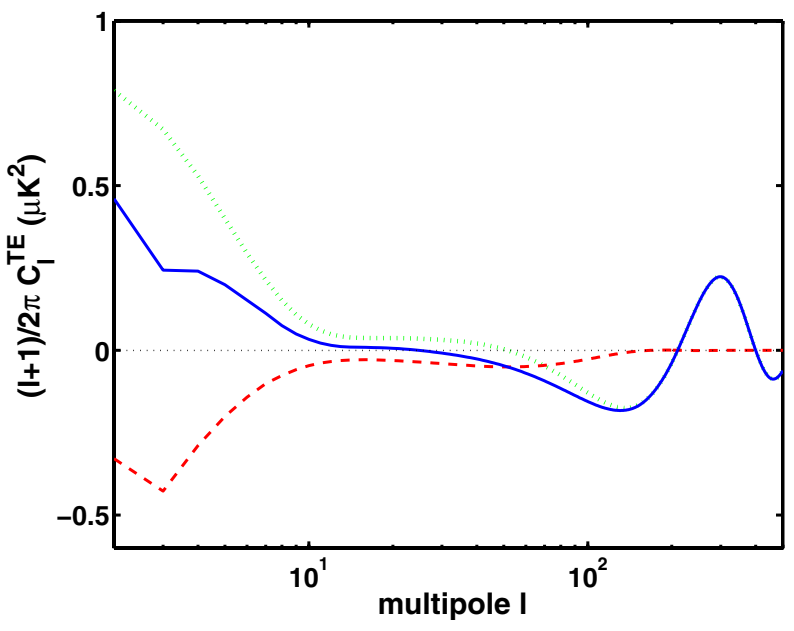

FIG. 12 (color online). The dotted line shows the contribution of density perturbations alone, and the dashed line shows the contribution of gravitational waves alone. The solid line is the sum of these contributions. It is seen from the graph that the inclusion of g.w. makes the total curve to be always below the d.p. curve.
We include the effects of reionization according to the model with $\tau_{\text {reion }}=0.09$. The (gw) contribution is numerically calculated from the solution to the integral Eq. (61), whereas the (dp) contribution is plotted according to the CMBfast code [18,31]. One can see from the graph that the negative $T E$ correlation function at lower $\ell$ 's is only possible if there is a significant amount of primordial gravitational waves. One can also see from the graph that a misinterpretation of the total $T E$ effect as being caused by density perturbations alone, could lead to a serious misestimation of $\tau_{\text {reion }}$.

The $T E$ correlation at lower $\ell$ 's measured by the WMAP mission $[9,48]$ shows clusters of data points, including the negative ones, that lie systematically below the theoretical curve based on density perturbations alone. It is true that the data points in the interval $10 \leqq \ell \leqq 70$ are concentrated near a zero level, the error bars are still large, and the measured $T E$ correlation can be appreciably different from the theoretical statistically averaged $T E$ correlation. However, the recent paper [9], page 26, explicitly emphasizes the detection of the $T E$ anticorrelation by WMAP: "The detection of the TE anticorrelation near $\ell \approx 30$ is a fundamental measurement of the physics of the formation of cosmological perturbations...". As we have already stated several times, the $T E$ anticorrelation at lower $\ell$ 's, such as $\ell \approx 30$, can only take place (within the framework of all other common assumptions) when a significant amount of relic gravitational waves is present.

Our theoretical position, as explained in this and previous papers, is such that we are asking not if relic gravitational waves exist, but where they are hiding in the presently available data. We shall now discuss some models that fit the CMB data and contain significant amounts of relic gravitational waves. More accurate observations with WMAP, and especially Planck, should firmly settle on the issue of the sign and value of the $T E$ correlation at lower multipoles. Hopefully, these observations will establish the presence of relic gravitational waves beyond reasonable doubts.

To sharpen the discussion of allowed parameters, we take the model with $\mathrm{n}=1.2, \tau_{\text {reion }}=0.09$ and $R=1$. We take the values $\Omega_{m} h^{2}=0.03, \Omega_{b} h^{2}=0.12, h=0.75$, and we normalize the g.w. contribution to $\frac{\ell(\ell+1)}{2 \pi} C_{\ell}^{T T}=$ $440 \mu \mathrm{K}^{2}$ at $\ell=2$. In Fig. 13(a) we show our calculation of the $T T$ correlation function in comparison with WMAP data and the best fit $\Lambda$ CDM model [8], [18]. (If it comes to the necessity of explaining "dark energy", natural modifications of general relativity will be superior to unnatural modifications of the matter sector [50].) One can see from the graph that even this model (which lies, arguably, on a somewhat extreme end) is consistent with the $T T$ data at all $\ell$ 's and significantly alleviates the much discussed tension between theory and experiment at $\ell=2$. In Fig. 13(b) we show the $T E$ correlation for exactly the same model. One can see that the inclusion of relic gravitational waves 

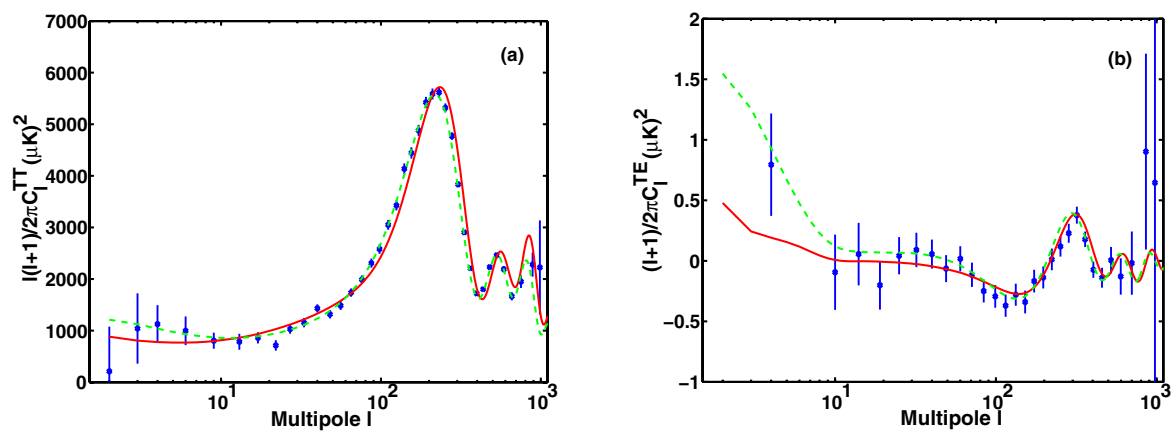

FIG. 13 (color online). The dashed line shows the best fit $\Lambda$ CDM model without gravitational waves. The solid line shows a model with spectral index $n=1.2$ and gravitational waves $R=1$.

makes more plausible the negative data points at lower $\ell$ 's. Since the relative contribution of gravitational waves becomes small at $\ell \gtrsim 90$, the higher $\ell$ portion of the graph is governed by density perturbations alone. Obviously, models with a little smaller $\mathrm{n}$ or $R$ reach the same goals.

Finally, in Fig. 14 we combine together all correlation functions induced by relic gravitational waves. The graphs are based on the discussed model with $\mathrm{n}=1.2, R=1$. The future detection of the $B B$ correlation will probably be the cleanest proof of presence of relic gravitational waves. For the discussed model, the predicted level of the $B B$ signal is $\left.\frac{\ell(\ell+1)}{2 \pi} C_{\ell}^{B B}\right|_{\ell \approx 90} \sim 0.1 \mu \mathrm{K}^{2}$ in the region near the first peak at $\ell \sim 90$. This level of $B$-mode polarization should be detectable by the experiments currently in preparation, such as CLOVER [51], BICEP [52] and others (see also [53]). Obviously, in this paper, we ignore many complications, including astrophysical foregrounds. More information can be found in Refs. [54-57].

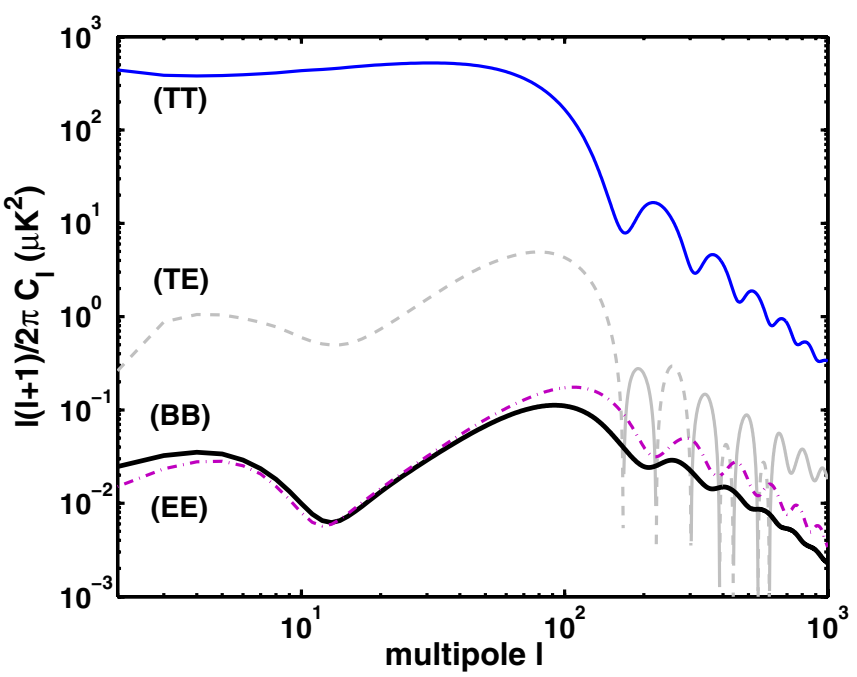

FIG. 14 (color online). The summary of CMB temperature and polarization anisotropies due to relic gravitational waves with $n=1.2$ and $R=1$.

\section{CONCLUSIONS}

In this paper, we summarized the properties of relic gravitational waves that are directly relevant to $\mathrm{CMB}$ calculations. We explained the reasons why we are working with g.w. backgrounds possessing specific statistical properties, amplitudes, spectral indices, etc. Then, we worked out, essentially from first principles, a theory of CMB anisotropies induced by relic gravitational waves. Some parts of this theory are rederivations and confirmations of previous studies, some parts are new. The important advantage of our approach, as we see it, is a transparent physical picture. We believe we have demonstrated in the paper that every detail of the derived correlation functions is under full analytical control. Clear understanding of the participating physical processes has led us to the conclusion that the $T E$ correlation in CMB can be a valuable probe of relic gravitational waves. We compared our theoretical findings with the WMAP data. We believe that the $T E$ anticorrelation detected by WMAP at $\ell \approx 30$ is certain evidence for relic gravitational waves in the already available data. We propose more accurate observations of the $T E$ correlation at lower $\ell$ 's and believe that these observations have the potential of providing a firm positive result.

\section{APPENDIX A: POLARIZATION STATES AND RANDOMNESS OF GRAVITATIONAL WAVES}

As stated above, the quantities $\left|s_{n}(\eta)\right|^{2}$ describe the magnitude of the mean-square fluctuations of the g.w. field in the corresponding polarization states $s$. We consider a particular mode $\mathbf{n}$ of the field:

$$
\begin{aligned}
h_{i j}(\mathbf{n}, \eta, \mathbf{x})= & \sum_{s=1,2} \stackrel{s}{p_{i j}}(\mathbf{n})\left(\stackrel{s}{h} h_{n}(\eta) e^{i \mathbf{n} \cdot \mathbf{x}} \stackrel{s}{\mathbf{n}}\right. \\
& \left.+\stackrel{s}{h}_{n}{ }^{*}(\eta) e^{-i \mathbf{n} \cdot \mathbf{x}^{s^{*}}} c_{\mathbf{n}}\right) .
\end{aligned}
$$

The lowest-order independent g.w. correlation functions amount to 


$$
\begin{aligned}
& \left\langle h_{i j}(\mathbf{n}, \eta, \mathbf{x}) \stackrel{s}{p}^{i j}(\mathbf{n}) h_{k l}\left(\mathbf{n}^{\prime}, \eta, \mathbf{x}^{\prime}\right) \stackrel{s^{\prime}}{p}\left(\mathbf{n}^{\prime}\right)\right\rangle \\
& =8\left|\stackrel{s}{h}_{n}(\eta)\right|^{2} \delta_{s s^{\prime}} \delta^{(3)}\left(\mathbf{n}-\mathbf{n}^{\prime}\right) .
\end{aligned}
$$

The action of the random g.w. field (A1) on free particles leads to their relative oscillatory motion. We refer this motion to a local inertial frame. Let a ring of free particles to lie in the $(\boldsymbol{l}, \mathbf{m})$-plane; the ring encircles the axis $\mathbf{n}$. Then, the mean-square amplitude of oscillations in the ' + ' polarization state is determined by $\left|h_{n}\right|^{2}$, whereas the mean-square amplitude of oscillations in the ' $X$ ' polarization state is determined by $\left|h_{n}^{2}\right|^{2}$. In general, the random gravitational-wave field can be such that the oscillation amplitudes $\left|\frac{1}{h_{n}}\right|^{2}$ and $\left|h_{n}\right|^{2}$ are different. But if $\left|h_{n}\right|^{2} \neq$ $\left|h_{n}^{2}\right|^{2}$, then the (averaged) observed picture of oscillations is not symmetric with respect to rotations around $\mathbf{n}$.

Formally, the correlation functions of the field with $\left|h_{n}\right|^{2} \neq\left|\stackrel{2}{h}_{n}\right|^{2}$ do not have symmetry with respect to the change of polarization basis. Indeed, the transition to the primed basis according to Eq. (7) brings the gravitationalwave mode (A1) to the form

$$
h_{i j}(\mathbf{n}, \eta, \mathbf{x})=\sum_{s=1,2} \stackrel{s}{p}_{i j}^{\prime}(\mathbf{n})\left(\stackrel{s}{b}{ }_{\mathbf{n}}(\eta) e^{i \mathbf{n} \cdot \mathbf{x}}+\stackrel{s}{b}_{\mathbf{n}}^{*}(\eta) e^{-i \mathbf{n} \cdot \mathbf{x}}\right),
$$

where

$$
\begin{aligned}
& \stackrel{1}{b}_{\mathbf{n}}(\eta)=\cos 2 \psi \stackrel{1}{h}_{n}(\eta) \stackrel{1}{c_{\mathbf{n}}}+\sin 2 \psi \stackrel{2}{h}{ }_{n}(\eta) \stackrel{2}{c_{\mathbf{n}},} \\
& \stackrel{2}{b}_{\mathbf{n}}(\eta)=-\sin 2 \psi \stackrel{1}{h}_{n}(\eta) \stackrel{1}{c_{\mathbf{n}}}+\cos 2 \psi \stackrel{2}{h}_{n n}(\eta){ }^{2} c_{\mathbf{n}} .
\end{aligned}
$$

Taking into account the relationships (16), we can now derive the correlation functions for the new polarization components:

$$
\begin{aligned}
1 & 1^{*} \\
\left\langle b_{\mathbf{n}} b_{\mathbf{n}^{\prime}}\right\rangle & =\left(\left|h_{n}\right|^{2} \cos ^{2} 2 \psi+\left|h_{n}\right|^{2} \sin ^{2} 2 \psi\right) \delta^{(3)}\left(\mathbf{n}-\mathbf{n}^{\prime}\right), \\
\left\langle b_{\mathbf{n}} b_{\mathbf{n}^{\prime}}^{*}\right\rangle & =\left(\left|h_{n}\right|^{2} \sin ^{2} 2 \psi+\left|h_{n}\right|^{2} \cos ^{2} 2 \psi\right) \delta^{(3)}\left(\mathbf{n}-\mathbf{n}^{\prime}\right), \\
\left\langle b_{\mathbf{n}} b_{\mathbf{n}^{\prime}}^{*}\right. & =-\left(\left|h_{n}\right|^{2}-\left|h_{n}\right|^{2}\right) \sin 2 \psi \cos 2 \psi \delta^{(3)}\left(\mathbf{n}-\mathbf{n}^{\prime}\right) .
\end{aligned}
$$

It is seen from (A3) that, in general, the $\psi$-dependence survives, and the assumption of statistical independence of polarization components in one basis is not equivalent to this assumption in another basis. However, one recovers the original correlation functions (A2) from (A3) if the conditions (18) are fulfilled.

Similar properties hold true for circular polarizations. A g.w. mode $h_{i j}(\mathbf{n}, \eta, \mathbf{x})$ expanded over circular polarization states is given by

$$
h_{i j}(\mathbf{n}, \eta, \mathbf{x})=\bar{h}_{i j}(\mathbf{n}, \eta, \mathbf{x})+\bar{h}_{i j}^{*}(\mathbf{n}, \eta, \mathbf{x}),
$$

where

$$
\bar{h}_{i j}(\mathbf{n}, \eta, \mathbf{x})=\sum_{s=L, R} \stackrel{s}{p}_{i j}(\mathbf{n}) \stackrel{s}{h}{ }_{n}(\eta) e^{i \mathbf{n} \cdot \mathbf{x}} c_{\mathbf{n}} \cdot
$$

We assume that the complex random coefficients $\stackrel{s}{c_{\mathbf{n}}}(s=$ $L, R)$, satisfy the statistical conditions (16).

The relevant independent correlation functions are calculated to be

$$
\begin{aligned}
& \left\langle\bar{h}_{i j}(\mathbf{n}, \eta, \mathbf{x}) \stackrel{s}{p}^{i j^{*}}(\mathbf{n}) \bar{h}_{k l}^{*}\left(\mathbf{n}^{\prime}, \eta, \mathbf{x}^{\prime}\right) \stackrel{s}{p}^{\prime k l}\left(\mathbf{n}^{\prime}\right)\right\rangle \\
& \quad=4\left|\stackrel{h}{h}_{n}(\eta)\right|^{2} \delta_{s s^{\prime}} \delta^{(3)}\left(\mathbf{n}-\mathbf{n}^{\prime}\right), \quad(s=L, R) .
\end{aligned}
$$

If the observer views the motion of test particles from the $\mathbf{- n}$ direction, i.e. against the direction of the incoming gravitational wave, the function $\left|h_{n}(\eta)\right|^{2}$ is responsible for the mean-square amplitude of the right-handed (clockwise) rotations of individual particles. The function $\left|h_{n}(\eta)\right|^{2}$ is responsible for the left-handed (counter-clockwise) rotations. (For more details about the motion of free particles in the field of gravitational waves, see [41].)

Expansion (A4) preserves its form under transformations (21), if one makes the replacements: $\stackrel{L \prime}{h}_{n}^{\prime}=\stackrel{h}{L}_{n} e^{i 2 \psi}$, $R_{n}^{\prime \prime}=h_{n} e^{-i 2 \psi}$. Therefore, the correlation functions (A5) do not change, regardless the value of the amplitudes $\left|\stackrel{s}{h}_{n}(\eta)\right|^{2}$ $(s=L, R)$. On the other hand, discrete transformations (22) generate the replacements: $\begin{array}{lll}L^{\prime} & h_{n}=R_{n}, & R^{\prime} \\ h_{n}\end{array}=h_{n}$. Therefore, the sense of correlation functions (A5) changes from $L$ to $R$ and vice versa. The symmetry between left and right is violated, unless the conditions (23) are fulfilled.

\section{APPENDIX B: ASTROPHYSICAL PREREQUISITES}

\section{Ionization history}

The ionization history of the Universe enters our equations through the density of free electrons $N_{e}(\eta)$, i.e. electrons available for Thompson scattering. Specifically, we operate with the quantity $q(\eta)$ :

$$
q(\eta)=\sigma_{T} a(\eta) N_{e}(\eta)
$$

The optical depth $\tau$ between some instant of time $\eta^{\prime}$ and a later instant $\eta$ is defined by the integral

$$
\tau\left(\eta, \eta^{\prime}\right)=\int_{\eta^{\prime}}^{\eta} d \eta^{\prime \prime} q\left(\eta^{\prime \prime}\right)
$$

The optical depth from some $\eta$ to the present time $\eta_{R}$ is denoted $\tau(\eta)$ and is given by

$$
\tau(\eta) \equiv \tau\left(\eta_{R}, \eta\right)=\int_{\eta}^{\eta_{R}} d \eta^{\prime} q\left(\eta^{\prime}\right)
$$

It follows from the above definitions that $\tau\left(\eta, \eta^{\prime}\right)=$ $\tau\left(\eta^{\prime}\right)-\tau(\eta)$ 
A key role in our discussion is played by the quantity $g(\eta)$ called the visibility function:

$$
g(\eta)=q(\eta) e^{-\tau(\eta)}=\frac{d}{d \eta} e^{-\tau(\eta)}, \quad \int_{0}^{\eta_{R}} g(\eta) d \eta=1
$$

The state of ionization is determined by microphysical processes during all the evolution of the Universe [58,59]. For our purposes it is sufficient to focus on two eras: early recombination and late reionization. The recombination of primordial plasma into atomic hydrogen and helium is accompanied by decoupling of CMB radiation from the rest of matter. (For simplicity, we do not distinguish here the notions of recombination and decoupling.) This relatively quick process has happened at redshifts around $z_{\mathrm{dec}} \approx 1100$. Much later, at redshifts around $z_{\text {reion }} \sim 10$ [9] the intergalactic medium has become ionized again, presumably, by ionizing radiation of first condensed objects.

The density of free electrons is modeled [60] by the expression

$$
N_{e}(\eta)=\left(1-\frac{Y_{p}}{2}\right) \frac{X_{e}(\eta) \Omega_{b} \rho_{c}}{m_{p}}\left(\frac{a\left(\eta_{R}\right)}{a(\eta)}\right)^{3},
$$

where $Y_{p} \approx 0.23$ is the primordial helium mass fraction, $X_{e}(\eta)$ is the fraction of ionized electrons, $\Omega_{b}$ is the baryon content, and $m_{p}$ is the mass of a proton. In the framework of linear perturbation theory it is sufficient to regard the electron density as homogeneous, i.e. depending only on $\eta$. For $X_{e}(\eta)$ we use the fitting formula [61]:
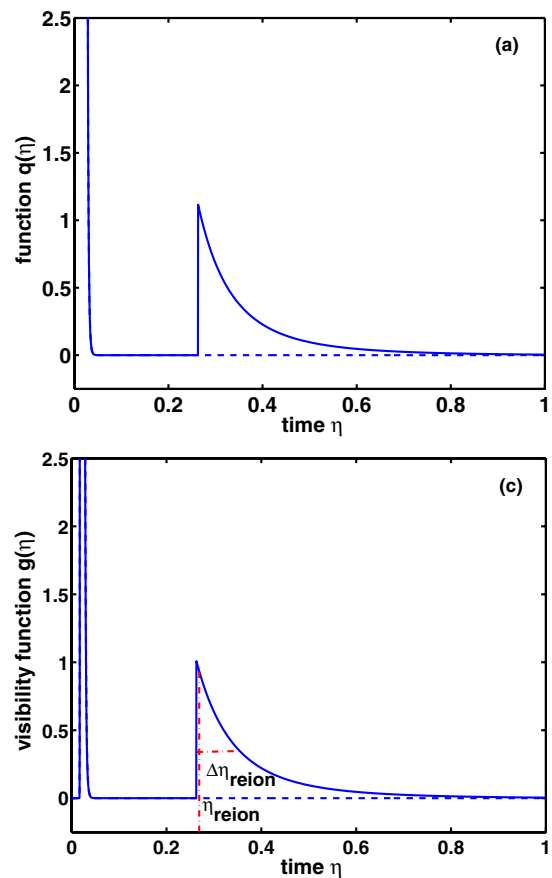

$$
\begin{aligned}
X_{e}(\eta)= & \left(1-\frac{Y_{p}}{2}\right)^{-1}\left(\frac{c_{2}}{1000}\right)\left(\frac{m_{p}}{2 \sigma_{T} l_{H} \rho_{c}}\right) \Omega_{b}^{c_{1}-1}\left(\frac{z}{1000}\right)^{c_{2}-1} \\
& \times\left(\frac{a^{\prime}}{a}\right)(1+z)^{-1},
\end{aligned}
$$

where $c_{1}=0.43, c_{2}=16+1.8 \ln \Omega_{B}$, and $z$ is a redshift.

As for the reionization, we assume that it was practically instantaneous and happened at a redshift $z_{\text {reion }} \approx 16$. The function $X_{e}$ is determined by Eq. (B2) for $z>z_{\text {reion }}$ and $X_{e}=1$ for $z \leq z_{\text {reion }}$.

To plot the graphs for $q(\eta), \tau(\eta)$ and $g(\eta)$ in Fig. 15, we use the above-mentioned parameters and $\Omega_{b}=0.046$ $[8,18]$. It is seen from Fig. 15 that the visibility function $g(\eta)$ is sharply peaked at the era of recombination. The peak can be characterized by the position of its maximum $\eta_{\mathrm{dec}}$, and the characteristic width $\Delta \eta_{\mathrm{dec}}$. A similar, but less pronounced, peak is present also at the era of reionization.

\section{Frequency dependence of the stokes parameters}

As is seen from Eq. (46), the frequency dependence of both, temperature and polarization, anisotropies is governed by the function $f(\tilde{\nu})$ [33]. We assume that the unperturbed radiation field has a black-body spectrum,

$$
n_{0}(\tilde{\nu})=\frac{1}{\exp (h \tilde{\nu} / k \tilde{T})-1},
$$

where $\tilde{T}=T a(\eta)$ and the present-day value of $T$ is $T\left(\eta_{R}\right) \approx 2.73 \mathrm{~K}$.

It follows from these formulas that $f(\tilde{\nu})$ is approximately 1 in the Reyleigh-Jeans part of the spectrum, and
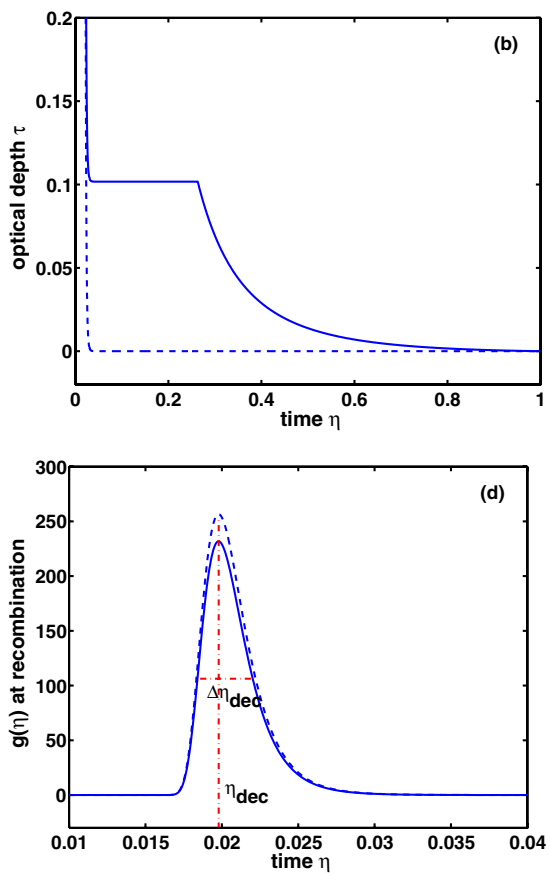

FIG. 15 (color online). The graphs (a), (b), (c) show the quantities $q(\eta), \tau(\eta), g(\eta)$. The graph (d) is a zooming of $g(\eta)$ in the region of recombination. The dashed line shows a model without reionization. The solid line takes reionization into account, with $\tau_{\text {reion }}=0.1$. 
$f(\tilde{\nu})$ varies as $h \tilde{\nu} / k \tilde{T}$ in the Wein part. In practice, we are usually interested in the total intensity, i.e. Stokes parameters integrated over all photon frequencies. This integration produces the factor $\gamma$ :

$$
\gamma \equiv \int d \tilde{\nu} \frac{h \tilde{\nu}^{3}}{c^{2}} n_{0}(\tilde{\nu}) f(\tilde{\nu})=-4 I_{0}
$$

where $I_{0}=\int d \tilde{\nu} \frac{h \tilde{\nu}^{3}}{c^{2}} n_{0}(\tilde{\nu})$ is the total intensity of the unperturbed radiation field. The factor $\gamma$ often appears in the main text of the paper.
APPENDIX C: TWO ESSENTIAL VARIABLES FOR TEMPERATURE AND POLARIZATION

It is seen from Eq. (48) that the frequency dependence of $\hat{\mathbf{n}}^{(1)}$ is determined by the factor $f(\tilde{\nu}) n_{0}(\tilde{\nu})$. Therefore, we can single out this factor and write

$$
\hat{\mathbf{n}}^{(\mathbf{1})}(\eta, \tilde{\nu}, \mu, \phi)=\frac{1}{2} f(\tilde{\nu}) n_{0}(\tilde{\nu}) \hat{\mathbf{n}}^{(\mathbf{1})}(\eta, \mu, \phi) .
$$

The equation that follows from (48) and (49) reads

$$
\left[\frac{\partial}{\partial \eta}+q(\eta)+i n \mu\right] \hat{\mathbf{n}}^{(\mathbf{1})}(\eta, \mu, \phi)=\left(1-\mu^{2}\right) e^{ \pm 2 i \phi} \frac{d h_{n}}{d \eta} \hat{\mathbf{u}}+\frac{q(\eta)}{4 \pi} \int_{-1}^{+1} \int_{0}^{2 \pi} d \mu^{\prime} d \phi^{\prime} \hat{\mathbf{P}}\left(\mu, \phi ; \mu^{\prime}, \phi^{\prime}\right) \hat{\mathbf{n}}^{(\mathbf{1})}\left(\eta, \mu^{\prime}, \phi^{\prime}\right) .
$$

The quantity $\hat{\mathbf{n}}^{(\mathbf{1})}(\eta, \mu, \phi)$ as a function of $\phi$ can be expanded in a series

$$
\hat{\mathbf{n}}^{(\mathbf{1})}(\eta, \mu, \phi)=\sum_{m=-\infty}^{+\infty} \hat{\mathbf{n}}_{m}^{(\mathbf{1})}(\eta, \mu) e^{i m \phi} .
$$

The explicit structure of the Chandrasekhar matrix $\hat{\mathbf{P}}\left(\mu, \phi ; \mu^{\prime}, \phi^{\prime}\right)$ is given by the expression [29] (equation (220) on p. 42):

$$
\hat{\mathbf{P}}\left(\mu, \phi ; \mu^{\prime}, \phi^{\prime}\right)=\mathbf{Q}\left[\mathbf{P}^{(\mathbf{0})}+\left(1-\mu^{2}\right)^{1 / 2}\left(1-\mu^{2}\right)^{1 / 2} \mathbf{P}^{(\mathbf{1})}+\mathbf{P}^{(2)}\right],
$$

where matrices $\mathbf{Q}, \mathbf{P}^{(\mathbf{0})}\left(\mu ; \mu^{\prime}\right), \mathbf{P}^{(\mathbf{1})}\left(\mu, \phi ; \mu^{\prime}, \phi^{\prime}\right), \mathbf{P}^{(2)}\left(\mu, \phi ; \mu^{\prime}, \phi^{\prime}\right)$ read

$$
\begin{aligned}
\mathbf{Q} & =\left(\begin{array}{lll}
1 & 0 & 0 \\
0 & 1 & 0 \\
0 & 0 & 2
\end{array}\right), \\
\mathbf{P}^{(\mathbf{0})} & =\frac{3}{4}\left(\begin{array}{ccc}
2\left(1-\mu^{2}\right)\left(1-\mu^{\prime 2}\right)+\mu^{2} \mu^{\prime 2} & \mu^{2} & 0 \\
\mu^{\prime 2} & 1 & 0 \\
0 & 0 & 0
\end{array}\right), \\
\mathbf{P}^{(\mathbf{1})} & =\frac{3}{4}\left(\begin{array}{ccc}
\mu \mu \mu^{\prime} \cos \left(\phi^{\prime}-\phi\right) & 0 & -2 \mu \sin \left(\phi^{\prime}-\phi\right) \\
0 & 0 & 0 \\
2 \mu^{\prime} \sin \left(\phi^{\prime}-\phi\right) & 0 & \cos \left(\phi^{\prime}-\phi\right)
\end{array}\right), \\
\mathbf{P}^{(2)} & =\frac{3}{4}\left(\begin{array}{ccc}
\mu^{2} \mu^{\prime 2} \cos 2\left(\phi^{\prime}-\phi\right) & -\mu^{2} \cos 2\left(\phi^{\prime}-\phi\right) & -\mu^{2} \mu^{\prime} \sin 2\left(\phi^{\prime}-\phi\right) \\
-\mu^{\prime 2} \cos 2\left(\phi^{\prime}-\phi\right) & \cos 2\left(\phi^{\prime}-\phi\right) & \mu^{\prime} \sin 2\left(\phi^{\prime}-\phi\right) \\
\mu \mu^{\prime 2} \sin 2\left(\phi^{\prime}-\phi\right) & -\mu \sin 2\left(\phi^{\prime}-\phi\right) & \mu \mu^{\prime} \cos 2\left(\phi^{\prime}-\phi\right)
\end{array}\right) .
\end{aligned}
$$

The structure of the Chandrasekhar matrix $\hat{\mathbf{P}}\left(\mu, \phi ; \mu^{\prime}, \phi^{\prime}\right)$ is such that it does not mix the $m \phi$-dependence, that is,

$$
\begin{aligned}
& \frac{1}{4 \pi} \int_{-1}^{+1} \int_{0}^{2 \pi} d \mu^{\prime} d \phi^{\prime} \hat{\mathbf{P}}\left(\mu, \phi ; \mu^{\prime}, \phi^{\prime}\right) \hat{\mathbf{n}}_{m}^{(\mathbf{1})}\left(\eta, \mu^{\prime}\right) e^{i m \phi^{\prime}} \\
& \quad \sim e^{i m \phi} .
\end{aligned}
$$

Moreover, this integral vanishes for all $|m|>2$. This means that Eq. (C1) is a homogeneous differential equation for all $\hat{\mathbf{n}}_{m}^{(\mathbf{1})}$ with $m \neq \pm 2$. Assuming zero initial conditions at some initial $\eta$, we obtain $\hat{\mathbf{n}}_{m}^{(\mathbf{1})}=0$ for all $m \neq \pm 2$. Hence, we are left with three functions of $(\eta, \mu)$ :

$$
\hat{\mathbf{n}}^{(\mathbf{1})}(\eta, \mu, \phi)=\hat{\mathbf{n}}^{(\mathbf{1})}(\eta, \mu) e^{ \pm 2 i \phi} .
$$

We are now able to show that only two out of the three functions $\hat{\mathbf{n}}^{(1)}(\eta, \mu)$ are independent. Indeed, using (C3) in Eq. (C1) we arrive at a system of three linear equations for the components of $\hat{\mathbf{n}}^{(\mathbf{1})}(\eta, \mu)$ : 


$$
\begin{aligned}
& \left(\frac{\partial}{\partial \eta}+q(\eta)+i n \mu\right)\left(\begin{array}{l}
\hat{n}_{1}(\eta, \mu) \\
\hat{n}_{2}(\eta, \mu) \\
\hat{n}_{3}(\eta, \mu)
\end{array}\right) \\
& \quad=\left(1-\mu^{2}\right) \frac{d h_{n}}{d \eta}\left(\begin{array}{l}
1 \\
1 \\
0
\end{array}\right)+\frac{3}{8} q(\eta)\left(\begin{array}{c}
\mu^{2} \\
-1 \\
\pm 2 i \mu
\end{array}\right) I(\eta)
\end{aligned}
$$

where $I(\eta)$ is the remaining integral that was left over from the last term in Eq. (C1):

$$
\begin{aligned}
I(\eta)= & \frac{1}{2} \int_{-1}^{+1} d \mu^{\prime}\left[\mu^{\prime 2} \hat{n}_{1}\left(\eta, \mu^{\prime}\right)-\hat{n}_{2}\left(\eta, \mu^{\prime}\right)\right. \\
& \left. \pm i \mu^{\prime} \hat{n}_{3}\left(\eta, \mu^{\prime}\right)\right] .
\end{aligned}
$$

Despite the complicated appearance, only two of the three Eqs. (C4) are really coupled. Indeed, making linear combinations of Eqs. (C4) it is easy to show that the combination $2 i \mu\left(\hat{n}_{1}-\hat{n}_{2}\right) \mp\left(1+\mu^{2}\right) \hat{n}_{3}$ satisfies a homogeneous differential equation. Assuming zero initial conditions, we derive

$$
2 i \mu\left(\hat{n}_{1}-\hat{n}_{2}\right) \mp\left(1+\mu^{2}\right) \hat{n}_{3}=0 .
$$

As two independent and essential variables we choose

$$
\begin{aligned}
& \alpha(\eta, \mu)=\frac{\hat{n}_{1}(\eta, \mu)+\hat{n}_{2}(\eta, \mu)}{\left(1-\mu^{2}\right)}, \\
& \beta(\eta, \mu)=\frac{\hat{n}_{1}(\eta, \mu)-\hat{n}_{2}(\eta, \mu)}{\left(1+\mu^{2}\right)}= \pm \frac{\hat{n}_{3}(\eta, \mu)}{2 i \mu} .
\end{aligned}
$$

In terms of $\alpha$ and $\beta$ equations of radiative transfer reduce to Eq. (51) and (52), and the definition (C5) for $I(\eta)$ takes the form of Eq. (53).

Although we have considered a gravitational-wave perturbation, the existence of only two essential variables is a general statement and it applies to density and rotational perturbations as well. In general, the perturbed radiative transfer equation contains an arbitrary function $f(\eta, \mu, \phi)$ in front of $\hat{\mathbf{u}}$, rather than a specific combination $\left(1-\mu^{2}\right) e^{ \pm 2 i \phi} d h_{n} / d \eta$ quoted in Eq. (C1). Function $f(\eta, \mu, \phi)$ can be expanded in a series similar to Eq. (C2):

$$
f(\eta, \mu, \phi)=\sum_{m=-\infty}^{+\infty} f_{m}(\eta, \mu) e^{i m \phi}
$$

Since the scattering integral (second term in the r.h.s of Eq. (C1)) vanishes for all $|m|>2$, the functions $\hat{\mathbf{n}}_{m}^{(\mathbf{1})}(\eta, \mu)$ are fully determined by $f_{m}(\eta, \mu)(|m|>2)$ and describe the temperature variations only. To discuss polarization, we have to consider three remaining cases $m=0, \pm 1, \pm 2$.

The explicit identification of the two essential variables $\alpha(\eta, \mu)$ and $\beta(\eta, \mu)$ for the case $m= \pm 2$ has been given above. Specifically for gravitational waves, $f_{2}=$ $\left(1-\mu^{2}\right) d h_{n}^{L}(\eta) / d \eta, f_{-2}=\left(1-\mu^{2}\right) d h_{n}^{R}(\eta) / d \eta$.

The identification of the two essential variables for the case $m=0$ proceeds in a similar manner. Having calcu- lated the scattering integral for $m=0$ one can show that the equation, analogous to Eq. (C4), will now read

$$
\begin{aligned}
& \left(\frac{\partial}{\partial \eta}+q(\eta)+i n \mu\right)\left(\begin{array}{c}
\hat{n}_{1}(\eta, \mu) \\
\hat{n}_{2}(\eta, \mu) \\
\hat{n}_{3}(\eta, \mu)
\end{array}\right) \\
& =\left(f_{0}(\eta, \mu)+q(\eta) I_{0}^{(1)}(\eta)\right)\left(\begin{array}{l}
1 \\
1 \\
0
\end{array}\right)+\frac{3}{8} q(\eta) \\
& \quad \times\left(\begin{array}{c}
3 \mu^{2}-2 \\
1 \\
0
\end{array}\right) \mathcal{J}(\eta),
\end{aligned}
$$

where

$$
\begin{aligned}
I_{0}^{(1)}(\eta) & =\frac{1}{4} \int_{-1}^{+1} d \mu^{\prime}\left[\hat{n}_{1}\left(\eta, \mu^{\prime}\right)+\hat{n}_{2}\left(\eta, \mu^{\prime}\right)\right], \\
\mathcal{J}(\eta) & =\frac{1}{3} \int_{-1}^{+1} d \mu^{\prime}\left[\left(3 \mu^{\prime 2}-2\right) \hat{n}_{1}\left(\eta, \mu^{\prime}\right)+\hat{n}_{2}\left(\eta, \mu^{\prime}\right)\right] .
\end{aligned}
$$

Obviously, $\hat{n}_{3}$ satisfies a homogeneous equation and can be put to zero. Specifically for density perturbations (see Appendix D), function $f_{0}(\eta, \mu)$ consists of terms representing gravitational-field perturbations and the Doppler term arising due to the baryon velocity:

$$
f_{0}(\eta, \mu)=\frac{1}{2}\left(\frac{d h}{d \eta}-\mu^{2} \frac{d h_{l}}{d \eta}+i q(\eta) \mu v_{b}(\eta)\right)
$$

We have checked that in the case $m= \pm 1$ the problem also reduces to only two essential variables. The combination $\hat{n}_{1}-\hat{n}_{2} \mp i \mu \hat{n}_{3}$ vanishes at the zero initial data.

\section{APPENDIX D: TEMPERATURE AND POLARIZATION ANISOTROPIES CAUSED BY DENSITY PERTURBATIONS}

To discover relic gravitational waves in the CMB data we have to distinguish their effects from the effects of density perturbations. The theory of temperature and polarization anisotropies caused by primordial density perturbations is very much similar to the theory of relic gravitational waves. We start from the metric Fourier expansion (10) with the polarization tensors (9). Having derived and solved integral equations of radiative transfer in the presence of density perturbations, we arrive at our final goal of distinguishing the $T E$ cross-correlations.

\section{Radiative transfer equations}

The equations of radiative transfer in the presence of a single mode $n$ of density perturbations are similar to Eq. (48) and read 


$$
\begin{aligned}
& {\left[\frac{\partial}{\partial \eta}+q(\eta)+i e^{i} n_{i}\right] \hat{\mathbf{n}}_{\mathbf{n}}^{(\mathbf{1})}\left(\eta, \tilde{\nu}, e^{i}\right)} \\
& =\frac{f(\tilde{\nu}) n_{0}(\tilde{\nu})}{2}\left[e^{i} e^{j} p_{i j}^{s}(\mathbf{n}) \frac{d h_{n}(\eta)}{d \eta}-q(\eta) e^{i} v_{i}\right] \hat{\mathbf{u}} \\
& \quad+\frac{q(\eta)}{4 \pi} \int d \Omega^{\prime} \hat{\mathbf{P}}\left(e^{i} ; e^{j j}\right) \hat{\mathbf{n}}_{\mathbf{n}}^{(\mathbf{1})}\left(\eta, \tilde{\nu}, e^{j j}\right),
\end{aligned}
$$

where the extra term $e^{i} v_{i}$ takes care of the movement of scattering electrons with respect to the chosen synchronous coordinate system [62-64].

For technical reasons, it is convenient to work with the "scalar" $h(\eta)$ and "longitudinal" $h_{l}(\eta)$ polarization mode functions, instead of the original $h_{n}(\eta)$ [65]. The relationship between them is

$$
{ }_{h n}^{1}(\eta)=\sqrt{\frac{3}{2}}\left(h(\eta)-h_{l}(\eta)\right), \quad \stackrel{2}{h}_{n}(\eta)=\sqrt{\frac{1}{3}} h_{l}(\eta),
$$

where the wave number index $n$ on $h(\eta)$ and $h_{l}(\eta)$ is implicit. Both polarization components of metric perturbations participate in Eq. (D1). In the frame associated with the density wave, i.e. for $\mathbf{n} / n=(0,0,1)$, the structures $e^{i} e^{j} p_{i j}^{s}$ (see Eq. (9)) and $e^{i} v_{i}=-i \mu v_{b}$ depend on $\mu=$ $\cos \theta$, but not on the azimuthal angle $\phi$.

By argumentation similar to that in Appendix C, one can show that a solution (not vanishing on zero initial data) to Eq. (D1) must have the form

$$
\begin{aligned}
\hat{\mathbf{n}}_{n}^{(1)}(\eta, \tilde{\nu}, \mu)= & \frac{f(\tilde{\nu}) n_{0}(\tilde{\nu})}{2}\left[\alpha_{n}(\eta, \mu)\left(\begin{array}{l}
1 \\
1 \\
0
\end{array}\right)\right. \\
& \left.+\beta_{n}(\eta, \mu)\left(\begin{array}{c}
1 \\
-1 \\
0
\end{array}\right)\right] .
\end{aligned}
$$

Substituting Eq. (D2) into Eq. (D1) we arrive at a system of coupled equations for $\alpha$ and $\beta$

$$
\begin{aligned}
& {\left[\frac{\partial}{\partial \eta}+q(\eta)+i n \mu\right] \alpha_{n}(\eta, \mu)=\frac{1}{2}\left(\frac{d h}{d \eta}-\mu^{2} \frac{d h_{l}}{d \eta}\right)+q(\eta)\left(I_{1}+i \mu v_{b}-\frac{1}{2} P_{2}(\mu) I_{2}\right),} \\
& {\left[\frac{\partial}{\partial \eta}+q(\eta)+i n \mu\right] \beta_{n}(\eta, \mu)=\frac{1}{2} q(\eta)\left(1-P_{2}(\mu)\right) I_{2},}
\end{aligned}
$$

where

$$
\begin{aligned}
& I_{1}(\eta)=\frac{1}{2} \int_{-1}^{+1} d \mu \alpha_{n}(\eta, \mu), \\
& I_{2}(\eta)=\frac{1}{2} \int_{-1}^{+1} d \mu\left[\left(1-P_{2}(\mu)\right) \beta_{n}(\eta, \mu)-P_{2}(\mu) \alpha_{n}(\eta, \mu)\right]
\end{aligned}
$$

The quantity $I_{1}$ is the monopole component of the perturbed radiation field, whereas $I_{2}$ is the quadrupole $(\ell=2)$ component, responsible for the generation of polarization.

To make contact with previous work we note that the variables $\alpha$ and $\beta$ are closely related to the variables $\Delta_{T}$ and $\Delta_{P}$ from Ref. [31]. Assuming a black body unperturbed radiation field, we have $\Delta_{T}=-\alpha, \Delta_{P}=-\beta$. We also note that the mode functions $h$ and $h_{l}$ are related to the mode functions $\mathrm{h}$ and $\eta$ used in [31,64] by $h=-2 \eta, h_{l}=-(\mathrm{h}+6 \eta)$. Keeping in mind the difference in notations, one can verify that Eqs. (D3a) and (D3b) are equivalent to equations (11) in Ref. [31].

\section{Integral equations and their solutions}

A formal solution to Eqs. (D3a) and (D3b) can be written as

$$
\begin{aligned}
& \alpha_{n}(\eta, \mu)=\int_{0}^{\eta} d \eta^{\prime} e^{-\tau\left(\eta, \eta^{\prime}\right)-i n \mu\left(\eta-\eta^{\prime}\right)}\left[\frac{1}{2}\left(\frac{d h}{d \eta}-\mu^{2} \frac{d h_{l}}{d \eta}\right)+q\left(I_{1}+i \mu v_{b}-\frac{1}{2} P_{2}(\mu) I_{2}\right)\right] \\
& \beta_{n}(\eta, \mu)=\frac{1}{2}\left(1-P_{2}(\mu)\right) \int_{0}^{\eta} d \eta^{\prime} e^{-\tau\left(\eta, \eta^{\prime}\right)-i n \mu\left(\eta-\eta^{\prime}\right)} I_{2}
\end{aligned}
$$

Proceeding in a manner similar to that in Sec. IV, we substitute (D4a) and (D5b) into (D4a) and (D4b). After certain rearrangements we arrive at two coupled integral equations for $I_{1}$ and $I_{2}$ : 


$$
\begin{aligned}
I_{1}(\eta)= & \int_{0}^{\eta} d \eta^{\prime} q\left(\eta^{\prime}\right) e^{-\tau\left(\eta, \eta^{\prime}\right)}\left[I_{1}\left(\eta^{\prime}\right) j_{0}(x)+\frac{1}{2} I_{2}\left(\eta^{\prime}\right) j_{2}(x)\right] \\
& +\int_{0}^{\eta} d \eta^{\prime} e^{-\tau\left(\eta, \eta^{\prime}\right)}\left[\frac{1}{2}\left(\frac{d h}{d \eta^{\prime}} j_{0}(x)+\frac{d h_{l}}{d \eta^{\prime}} \frac{d^{2} j_{0}}{d x^{2}}\right)+q\left(\eta^{\prime}\right) v_{b}\left(\eta^{\prime}\right) j_{1}(x)\right], \\
I_{2}(\eta)= & \int_{0}^{\eta} d \eta^{\prime} q\left(\eta^{\prime}\right) e^{-\tau\left(\eta, \eta^{\prime}\right)}\left[I_{1}\left(\eta^{\prime}\right) j_{2}(x)+I_{2}\left(\eta^{\prime}\right)\left(\left(\frac{18}{x^{2}}-\frac{1}{2}\right) j_{2}(x)-\frac{3}{2 x} j_{1}(x)\right)\right] \\
& +\int_{0}^{\eta} d \eta^{\prime} e^{-\tau\left(\eta, \eta^{\prime}\right)}\left[\frac{1}{2}\left(\frac{d h}{d \eta^{\prime}} j_{2}(x)+\frac{d h_{l}}{d \eta^{\prime}}\left(j_{2}(x)+3 \frac{d^{2} j_{0}}{d x^{2}}\right)\right)+q\left(\eta^{\prime}\right) v_{b}\left(\eta^{\prime}\right) \frac{d j_{2}}{d x}\right],
\end{aligned}
$$

where the argument of spherical Bessel functions $j_{\ell}(x)$ is $x=n\left(\eta-\eta^{\prime}\right)$.

Eqs. (D6a) and (D6b) together with the continuity equations for matter perturbations and Einstein equations for metric perturbations (see for example [64,65]), form a closed system of coupled integro-differential equations. In previous treatments $[31,63,64]$, the radiative transfer equations were presented as an infinite series of coupled ordinary differential equations.

Similarly to what was done in Sec. IV B, we can analyze Eqs. (D6a) and (D6b) in terms of expansions in powers of $n$,

$$
I_{1}=\sum_{k=0}^{\infty} I_{1}^{(k)} n^{2 k}, \quad I_{2}=\sum_{k=0}^{\infty} I_{2}^{(k)} n^{2 k}
$$

The kernels of Eqs. (D6a) and (D6b) are expanded in powers of $x$. In the long-wavelength approximation, i.e. for $x \ll 1$, we can limit ourselves by first nonvanishing terms. The zero-order approximation brings us to the equations

$$
\begin{aligned}
I_{1}^{(0)}(\eta)= & \int_{0}^{\eta} d \eta^{\prime} q\left(\eta^{\prime}\right) e^{-\tau\left(\eta, \eta^{\prime}\right)} I_{1}^{(0)}\left(\eta^{\prime}\right) \\
& +\int_{0}^{\eta} d \eta^{\prime} e^{-\tau\left(\eta, \eta^{\prime}\right)}\left[\frac{1}{2}\left(\frac{d h}{d \eta^{\prime}}-\frac{1}{3} \frac{d h_{l}}{d \eta^{\prime}}\right)\right. \\
& \left.+\frac{1}{3} q\left(\eta^{\prime}\right) v_{b}\left(\eta^{\prime}\right) x\right] \\
I_{2}^{(0)}(\eta)= & \frac{7}{10} \int_{0}^{\eta} d \eta^{\prime} q\left(\eta^{\prime}\right) e^{-\tau\left(\eta, \eta^{\prime}\right)} I_{2}^{(0)}\left(\eta^{\prime}\right)+\frac{1}{15} \\
& \times \int_{0}^{\eta} d \eta^{\prime} e^{-\tau\left(\eta, \eta^{\prime}\right)}\left[-\frac{d h_{l}}{d \eta^{\prime}}+2 q\left(\eta^{\prime}\right) v_{b}\left(\eta^{\prime}\right) x\right] .
\end{aligned}
$$

The solution to these equations is given by

$$
\begin{aligned}
I_{1}^{(0)}(\eta)= & \int_{0}^{\eta} d \eta^{\prime}\left[\frac{1}{2}\left(\frac{d h}{d \eta^{\prime}}-\frac{1}{3} \frac{d h_{l}}{d \eta^{\prime}}\right)\right. \\
& \left.+\frac{1}{3} n \int_{0}^{\eta^{\prime}} d \eta^{\prime \prime} q\left(\eta^{\prime \prime}\right) e^{-\tau\left(\eta^{\prime}, \eta^{\prime \prime}\right)} v_{b}\left(\eta^{\prime \prime}\right)\right], \quad \text { (D8a) } \\
I_{2}^{(0)}(\eta)= & \frac{1}{15} \int_{0}^{\eta} d \eta^{\prime} e^{-(3 / 10) \tau\left(\eta, \eta^{\prime}\right)} \\
& \times\left[-\frac{d h_{l}}{d \eta^{\prime}}+2 n \int_{0}^{\eta^{\prime}} d \eta^{\prime \prime} q\left(\eta^{\prime \prime}\right) e^{-\tau\left(\eta^{\prime}, \eta^{\prime \prime}\right)} v_{b}\left(\eta^{\prime \prime}\right)\right] .
\end{aligned}
$$

\section{Multipole coefficients}

We are mostly interested in the present-day values of $\alpha$ and $\beta$ and, hence, we put $\eta=\eta_{R}$ in (D5a) and (D5b). Irrespective of approximation in which the functions $I_{1}(\eta), I_{2}(\eta)$ are known, the multipole coefficients for the radiation field can be found in a way similar to that in Sec. V:

$a_{\ell m}^{T}(n)=(-i)^{\ell} \gamma \sqrt{4 \pi(2 \ell+1)} \delta_{m 0} a_{\ell}^{T}(n)$,

$a_{\ell m}^{E}(n)=(-i)^{\ell} \gamma \sqrt{4 \pi(2 \ell+1)} \delta_{m 0} a_{\ell}^{E}(n), \quad a_{\ell m}^{B}(n)=0$,

where $a_{\ell}^{T}(n), a_{\ell}^{E}(n)$ are given by

$$
\begin{aligned}
a_{\ell}^{T}(n)= & \int_{0}^{\eta_{R}} d \eta\left[e^{-\tau}\left(\frac{1}{2} \frac{d h}{d \eta}+\frac{1}{2} \frac{d h_{l}}{d \eta} \frac{d^{2}}{d \zeta^{2}}\right)\right. \\
& \left.+g(\eta)\left(I_{1}+v_{b} \frac{d}{d \zeta}-\frac{3}{4} I_{2}\left(1+\frac{d^{2}}{d \zeta^{2}}\right)\right)\right] j_{\ell}(\zeta)
\end{aligned}
$$

$a_{\ell}^{E}(n)=\frac{3}{4}\left(\frac{(\ell+2) !}{(\ell-2) !}\right)^{1 / 2} \int_{0}^{\eta_{R}} d \eta g(\eta) I_{2} \frac{j_{\ell}(\zeta)}{\zeta^{2}}$

and $\zeta=n\left(\eta_{R}-\eta\right)$. As expected, in the case of density perturbations all $a_{\ell m}=0$ for $m \neq 0$ and $a_{\ell m}^{B}(n)=0$, $[31,32,66,67]$. The formal reason for this is that all quantities in (D2) do not depend on $\phi$ (A more detailed exposition can be found in $[31,32])$.

Let us start from the temperature multipoles. For illustration, we consider an instantaneous recombination. We replace $e^{-\tau}$ with the step function, $g(\eta)$ with the $\delta$-function, and we neglect the $I_{2}$ term. Then, we get

$$
\begin{aligned}
a_{\ell}^{T}(n)= & {\left.\left[\left(I_{1}+v_{b} \frac{d}{d \zeta}\right) j_{\ell}(\zeta)\right]\right|_{\eta=\eta_{\mathrm{dec}}} } \\
& +\frac{1}{2} \int_{\eta_{\mathrm{dec}}}^{\eta_{R}} d \eta\left(\frac{d h}{d \eta}+\frac{d h_{l}}{d \eta} \frac{d^{2}}{d \zeta^{2}}\right) j_{\ell}(\zeta) .
\end{aligned}
$$

This expression can be further simplified by taking the remaining integral by parts. After some rearrangements we arrive at the final expression 


$$
\begin{aligned}
a_{\ell}^{T}(n)= & \left.\left(\frac{1}{2 n^{2}} \frac{d^{2} h_{l}}{d \eta^{2}} \delta_{\ell 0}+\frac{1}{6 n} \frac{d h_{l}}{d \eta} \delta_{\ell 1}\right)\right|_{\eta=\eta_{R}} \\
& +\left[\left(-\frac{1}{2 n^{2}} \frac{d^{2} h_{l}}{d \eta^{2}}+I_{1}+\left(-\frac{1}{2 n} \frac{d h_{l}}{d \eta}+v_{b}\right) \frac{d}{d \zeta}\right)\right. \\
& \left.\times j_{\ell}(\zeta)\right]\left.\right|_{\eta=\eta_{\mathrm{dec}}} \\
& +\frac{1}{2} \int_{\eta_{\mathrm{dec}}}^{\eta_{R}} d \eta\left(\frac{d h}{d \eta}+\frac{1}{n^{2}} \frac{d^{3} h_{l}}{d \eta^{3}}\right) j_{\ell}(\zeta) .
\end{aligned}
$$

For the growing mode of density perturbations in the matter-dominated era, the integrand of the remaining integral vanishes. If, in addition, the intrinsic temperature perturbation $I_{1}$ and the plasma velocity $v_{b}$ are zero at $\eta=$ $\eta_{d e c}$, we recover from Eq. (D10) the four terms of the full Sachs-Wolfe formula (43) in Ref. [68]. We would like to note in passing that the origin of the often used, including this paper, combination $\ell(\ell+1) C_{\ell}$ is in fact a historical accident, when a wrong motivation leads to a convenient notation. This combination arises in the essentially incorrect formula $\ell(\ell+1) C_{\ell}=$ const that can be derived after the unjustified neglect of three out of four terms in the original Sachs-Wolfe formula (43) in Ref. [68]. This clarification is important not only by itself, but also for correct physical interpretation of reasons for the rise toward the "first peak" at $\ell \approx 220$ of the actually observed function $\ell(\ell+1) C_{\ell}$. For more details, see [69].

We now turn to polarization anisotropies. We use the zero-order approximation (D8b) in (D9b),

$$
\begin{aligned}
a_{\ell}^{E}= & \frac{1}{20}\left(\frac{(\ell+2) !}{(\ell-2) !}\right)^{1 / 2} \int_{0}^{\eta_{R}} d \eta q e^{-(7 / 10) \tau} \frac{j_{\ell}(\zeta)}{\zeta^{2}} \\
& \times \int_{0}^{\eta} d \eta^{\prime} e^{-(3 / 10) \tau\left(\eta^{\prime}\right)}\left(-\frac{d h_{l}}{d \eta^{\prime}}\right. \\
& \left.+2 n \int_{0}^{\eta^{\prime}} d \eta^{\prime \prime} q\left(\eta^{\prime \prime}\right) e^{-\tau\left(\eta^{\prime}, \eta^{\prime \prime}\right)} v_{b}\left(\eta^{\prime \prime}\right)\right)
\end{aligned}
$$

As $g(\eta)$ is a narrow function, the integrand is localized near $\eta=\eta_{\text {dec }}$. By the same argumentation that was used in Sec. VIB, the above expression can be reduced to quantities evaluated at $\eta=\eta_{\mathrm{dec}}$,

$\left.a_{\ell}^{E}(n) \approx \frac{1}{20}\left(\frac{(\ell+2) !}{(\ell-2) !}\right)^{1 / 2}\left[\frac{j_{\ell}(\zeta)}{\zeta 2}\left(-\frac{d h_{l}}{d \eta} \Delta+2 n v_{b} \tilde{\Delta}\right)\right]\right|_{\eta=\eta_{\mathrm{dec}}}$,

(D11)

where the factors $\Delta$ and $\tilde{\Delta}$ are of the order of the width of $g(\eta)$ in the recombination era. Explicitly, they are given by

$$
\begin{aligned}
\Delta= & \int_{0}^{\eta_{R}} d \eta g(\eta) \int_{0}^{\eta} d \eta^{\prime} e^{-(3 / 10) \tau\left(\eta, \eta^{\prime}\right)}, \\
\tilde{\Delta}= & \int_{0}^{\eta_{R}} d \eta g(\eta) \\
& \times \int_{0}^{\eta} d \eta^{\prime} e^{-(3 / 10) \tau\left(\eta, \eta^{\prime}\right)} \int_{0}^{\eta^{\prime}} d \eta^{\prime \prime} q\left(\eta^{\prime \prime}\right) e^{-\tau\left(\eta^{\prime}, \eta^{\prime \prime}\right)} .
\end{aligned}
$$

The angular power spectra in the case of density perturbations are described by formulas analogous to (86). By the steps similar to those in Sec. V C it can be shown that the power spectra are given by

$$
C_{\ell}^{X X^{\prime}}=\frac{\mathcal{C}^{2}}{\pi} \int n d n a_{\ell}^{X}(n) a_{\ell}^{X^{\prime}}(n),
$$

where $\mathcal{C}$ for density perturbations is $\mathcal{C}=\sqrt{24} \pi l_{P l}$.

\section{Temperature-polarization correlations}

We focus on the $T E$ correlation at the relatively small multipoles, $\ell \lesssim 70$. The dominant contribution to these multipoles comes from density perturbations that did not enter the Hubble radius at recombination, $n \lesssim 70$. It is sufficient to consider the early time evolution of these perturbations in the matter-dominated era.

Restricting our analysis to the growing mode, we write for the metric and matter perturbations (see, for example, [65]):

$$
\begin{array}{rlrl}
h(\eta) & =B_{n}=\mathrm{const}, & h_{l}(\eta) & =B_{n}(n \eta)^{2}, \\
I_{1}^{(0)}=\frac{\delta T}{T}=-\frac{2}{3} B_{n}(n \eta)^{2}, & v_{b} & =-\frac{2}{9} B_{n}(n \eta)^{3} .
\end{array}
$$

Substituting this solution into (D10) and (D11) and taking into account only the lowest-order terms in $n$ (the longwavelength approximation) we arrive at

$$
\begin{aligned}
& a_{\ell}^{T} \approx-\left.B_{n} j_{\ell}(\zeta)\right|_{\eta=\eta_{\mathrm{dec}}} \\
& a_{\ell}^{E} \approx-\left.\frac{1}{10} n^{2} B_{n} \eta_{\operatorname{dec}} \Delta\left(\frac{(\ell+2) !}{(\ell-2) !}\right)^{1 / 2}\left(\frac{j_{\ell}(\zeta)}{\zeta^{2}}\right)\right|_{\eta=\eta_{\mathrm{rec}}} .
\end{aligned}
$$

Finally, from Eq. (D12) we obtain for the $T E$ correlation:

$$
\left.C_{\ell}^{T E} \approx \frac{\mathcal{C}^{2}}{\pi} \frac{\eta_{\mathrm{dec}} \Delta}{10}\left(\frac{(\ell+2) !}{(\ell-2) !}\right)^{1 / 2} \int d n n^{3} B_{n}^{2}\left(\frac{j_{\ell}(\zeta)}{\zeta}\right)^{2}\right|_{\eta=\eta_{\mathrm{dec}}} .
$$

All terms in the above expression are strictly positive. Therefore, the $T E$ correlation caused by density perturbations, in contrast to the case of gravitational waves, must be positive at lower multipoles $\ell \leqq 50$ where our approximations are still valid. As mentioned before, this difference between gravitational waves and density perturbations boils down to the difference in the sign of first timederivative of the associated metric perturbations. 
[1] J. Bock et al., astro-ph/0604101.

[2] L. P. Grishchuk, Zh. Eksp. Teor. Fiz. 67, 825 (1974) [Sov. Phys. JETP 40, 409 (1975)]; Ann. N.Y. Acad. Sci. 302, 439 (1977); Pis'ma Zh. Eksp. Teor. Fiz. 23, 326 (1976) [JETP Lett. 23, 293 (1976)].

[3] Ya. B. Zel'dovich and A. A. Starobinsky, Zh. Eksp. Teor. Fiz. 61, 2161 (1971) [Sov. Phys. JETP 34, 1159 (1972)].

[4] L.P. Grishchuk and A. G. Polnarev, in: General Relativity and Gravitation, 100 Years After the Birth of A. Einstein, edited by A. Held (Plenum Press, NY, 1980), Vol. 2, p. 393.

[5] L.P. Grishchuk, Usp. Fiz. Nauk 175, 1289 (2005) [Phys. Usp. 48, 1235 (2005)].

[6] V. N. Lukash, Usp. Fiz. Nauk 176, 113 (2006) [Phys. Usp. 49 (2006)].

[7] H. V. Peiris et al., Astrophys. J. Suppl. Ser. 148, 213 (2003).

[8] D. N. Spergel et al., astro-ph/0603449.

[9] L. Page et al., astro-ph/0603450.

[10] Planck Collaboration, astro-ph/0604069.

[11] S. Bose and L. P. Grishchuk, Phys. Rev. D 66, 043529 (2002).

[12] E. Newman and R. Penrose, J. Math. Phys. (N.Y.) 7, 863 (1966).

[13] J. N. Goldberg et al., J. Math. Phys. (N.Y.) 8, 2155 (1967).

[14] K. S. Thorne, Rev. Mod. Phys. 52, 299 (1980).

[15] Y. Watanabe and E. Komatsu, Phys. Rev. D 73, 123515 (2006).

[16] L. P. Grishchuk and M. Solokhin, Phys. Rev. D 43, 2566 (1991).

[17] L.P. Grishchuk, LNP Vol. 562: Gyros, Clocks, Interferometers ..., Testing Relativistic Gravity in Space 167 (2001).

[18] Legacy Archive for Microwave Background Data Analysis (LAMBDA), http://lambda.gsfc.nasa.gov.

[19] G. Hinshaw et al., astro-ph/0603451.

[20] L.P. Grishchuk, Usp. Fiz. Nauk 156, 297 (1988) [Sov. Phys. Usp. 31, 940 (1988)].

[21] B. Allen and J.D. Romano, Phys. Rev. D 59, 102001 (1999).

[22] V. Corbin and N.J. Cornish, Classical Quantum Gravity 23, 2435 (2006).

[23] L. A. Boyle and P. J. Steinhardt, astro-ph/0512014.

[24] Asantha Cooray, Mod. Phys. Lett. A 20, 2503 (2005).

[25] T. L. Smith, M. Kamionkowski, and A. Cooray, Phys. Rev. D 73, 023504 (2006).

[26] T. Smith, H. Peiris, and A. Cooray, Phys. Rev. D 73, 123503 (2006).

[27] S. Chongchitnan and G. Efstathiou, astro-ph/0603118.

[28] V. Mandic and A. Buonanno, Phys. Rev. D 73, 063008 (2006).

[29] S. Chandrasekhar, Radiative Transfer (Dover Publications Inc., New York, 1960).

[30] L. D. Landau and E. M. Lifshitz, The Classical Theory of Fields (Pergamon Press, Oxford, 1975), 4th rev. engl. ed..

[31] M. Zaldarriaga and U. Seljak, Phys. Rev. D 55, 1830 (1997).

[32] M. Kamionkowski, A. Kosowsky, and A. Stebbins, Phys. Rev. D 55, 7368 (1997).
[33] M. M. Basko and A. G. Polnarev, Sov. Astron. 24, 268 (1980).

[34] A. G. Polnarev, Sov. Astron. 29, 607 (1985).

[35] R. W. Lindquist, Ann. Phys. (N.Y.) 37, 487 (1966).

[36] N. Caderni et al., Phys. Rev. D 17, 1901 (1978).

[37] P. Naselsky, D. Novikov, and I. Novikov, Adv. Space Res. 31, 417 (2003).

[38] R. Crittenden et al., Phys. Rev. Lett. 71, 324 (1993).

[39] U. Seljak and M. Zaldarriaga, Phys. Rev. Lett. 78, 2054 (1997).

[40] R. A. Frewin, A. G. Polnarev, and P. Coles, Mon. Not. R. Astron. Soc. 266, L21 (1994).

[41] D. Baskaran and L.P. Grishchuk, Classical Quantum Gravity 21, 4041 (2004).

[42] A.N. Varshalovich, D. A. Moskalev, and V.K. Khersonskii, Quantum Theory of Angular Momentum (World Scientific, Singapore, 1988).

[43] L. P. Grishchuk and J. Martin, Phys. Rev. D 56, 1924 (1997).

[44] A. Lue, L. Wang, and M. Kamionkowski, Phys. Rev. Lett. 83, 1506 (1999).

[45] B. Allen and S. Koranda, Phys. Rev. D 50, 3713 (1994).

[46] J. R. Pritchard and M. Kamionkowski, Ann. Phys. (N.Y.) 318, 2 (2005);

[47] W. Zhao and Y. Zhang, astro-ph/0508345.

[48] A. Kogut et al., Astrophys. J. Suppl. Ser. 148, 161 (2003).

[49] B. Keating and N. Miller, New Astron. Rev. 50, 184 (2006).

[50] S. V. Babak and L. P. Grishchuk, Int. J. Mod. Phys. D 12, 1905 (2003).

[51] B. Maffei et al., astro-ph/0407148.

[52] B. G. Keating et al., in Proceedings of the SPIE, edited by S. Fineschi (SPIE Press, 2003), Vol. 4843, p. 284.

[53] B. Keating, A. G. Polnarev, N. Miller, and D. Baskaran, Int. J. Mod. Phys. A 21, 2459 (2006).

[54] B. Keating et al., Astrophys. J. 495, 580 (1998).

[55] M. V. Sazhin, G. Sironi, and O.S. Khovanskaya, New Astron. Rev. 9, 83 (2004).

[56] P.D. Naselsky, I.D. Novikov, and L.-Y. Chiang, Astrophys. J. 642, 617 (2006).

[57] A. Lewis and A. Challinor, Phys. Rep. 429, 1 (2006).

[58] P. D. Naselskii and A. G. Polnarev, Astrofizika 26, 543 (1987) [Astrophysics (Engl. Transl.) 26, 327 (1987)].

[59] A. G. Doroshkevich, I. P. Naselsky, P. D. Naselsky, and I. D. Novikov, Astrophys. J. 586, 709 (2003).

[60] P.J.E. Peebles, Principles of Physical Cosmology (Princeton University Press, Princeton, 1993).

[61] W. Hu and N. Sugiyama, Astrophys. J. 444, 489 (1995).

[62] N. Kaiser, Mon. Not. R. Astron. Soc. 202, 1169 (1983).

[63] J. R. Bond and G. Efstathiou, Astrophys. J. Lett. 285, L45 (1984).

[64] C.-P. Ma and E. Bertschinger, Astrophys. J. 455, 7 (1995).

[65] L. P. Grishchuk, Phys. Rev. D 50, 7154 (1994).

[66] U. Seljak, Astrophys. J. 482, 6 (1997).

[67] W. Hu and M. White, New Astron. Rev. 2, 323 (1997).

[68] R. K. Sachs and A.M. Wolfe, Astrophys. J. 147, 73 (1967).

[69] A. Dimitropoulos and L. P. Grishchuk, Int. J. Mod. Phys. D 11, 259 (2002) 